\title{
Search for Chargino-Neutralino Pair Production with the DZero Detector at the Tevatron
}

\author{
A Dissertation Presented \\ by \\ Zhongmin Wang \\ to \\ The Graduate School \\ in Partial Fulfillment of the Requirements \\ for the Degree of \\ Ph.D. \\ in \\ Physics
}

Stony Brook University

May 2004 
Copyright (c) by Zhongmin Wang 2004 


\section{State University of New York at Stony Brook \\ The Graduate School \\ Zhongmin Wang}

We, the dissertation committee for the above candidate for the Ph.D. degree, hereby recommend acceptance of the dissertation.

\begin{tabular}{c}
\hline dissertation director \\
Dr. Michael Rijssenbeek \\
Department of Physics and Astronomy \\
\hline chairman of defense \\
Dr. Jack Smith \\
Department of Physics and Astronomy \\
\hline committee member \\
Dr. Barbara Jacak \\
Department of Physics and Astronomy \\
\hline outside member \\
Dr. Serban Protopopescu \\
Physics Department \\
Brookhaven National Laboratory
\end{tabular}

This dissertation is accepted by the Graduate School.

Graduate School 


\title{
Abstract of the Dissertation Search for Chargino-Neutralino Pair Production with the DZero Detector at the Tevatron
}

\author{
by \\ Zhongmin Wang \\ Ph.D. \\ in
}

Physics

Stony Brook University

2004

We have searched for evidence for the chargino $\left(\tilde{\chi}_{1}^{ \pm}\right)$and neutralino $\left(\tilde{\chi}_{2}^{0}\right)$ pair production in proton anti-proton collisions at a center of mass energy of $1.96 \mathrm{TeV}$ with the $\mathrm{D} \varnothing$ detector at the Fermilab Tevatron collider. Data corresponding to an integrated luminosity of $124.5 \mathrm{pb}^{-1}$ were examined for events containing likesign electron pair and missing energy for the first time at $D \varnothing$. We observed no excess above the yield from Standard Model processes. In the framework of mSUGRA, we set a series of upper limits, at the $95 \%$ confidence level, of the chargino neutralino production cross section times the branching fraction to tri-electrons 
as a function of the chargino mass. These limits range from 0.79

$\mathrm{pb}$ for $m_{\tilde{\chi}_{1}^{ \pm}}=86.9 \mathrm{GeV} / \mathrm{c}^{2}$ to $0.52 \mathrm{pb}$ for $m_{\tilde{\chi}_{1}^{ \pm}}=115.1 \mathrm{GeV} / \mathrm{c}^{2}$. 
To my parents and my wife 


\section{Contents}

$\begin{array}{ll}\text { Acknowledgements } & \text { xvi }\end{array}$

1 Introduction 1

1.1 The Standard Model . . . . . . . . . . . . . . . . 1

1.2 The Problems of the Standard Model . . . . . . . . . . . 3

1.3 Supersymmetry . . . . . . . . . . . . . . 5

1.3.1 Minimal Supersymmetric Standard Model . . . . . . 5

1.3.2 R-parity and LSP ............... 7

1.3.3 Minimal Supergravity . . . . . . . . . . 8

1.4 Search for $\tilde{\chi}_{1}^{ \pm} \tilde{\chi}_{2}^{0}$ in the Like-Sign Dilepton channel . . . . . . . 10

2 Experimental Apparatus $\quad 14$

2.1 The Fermilab Accelerators . . . . . . . . . . . . . 14

2.1.1 Pre-Accelerator, LINAC, and the Booster . . . . . 15

2.1.2 The Main Injector and Antiproton Production . . . . . 17

2.1.3 The Tevatron .................. 19

2.2 The DØ Coordinate System . . . . . . . . . . . 19

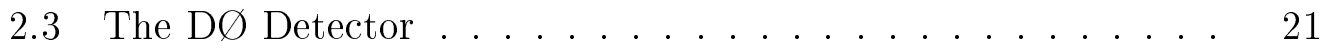


2.3.1 Central Tracking System . . . . . . . . . 23

2.3.2 Preshower Detectors ............ 30

2.3.3 The Calorimeter System ........... 35

2.3.4 Muon Detector ................ 44

3 Data Acquisition and Event Reconstruction 46

3.1 DØ Trigger and Data Acquisition . . . . . . . . . . 46

3.1.1 Level 0 and the Luminosity Monitor . . . . . . . 47

3.1.2 Level $1 \ldots \ldots \ldots \ldots$. . . . . . . . . . . 48

3.1.3 Level 2........................ 48

3.1.4 Level 3 and Data Acquisition .......... 50

3.2 Offline Event Reconstruction . . . . . . . . . . . 50

3.3 Track Reconstruction ................. 52

3.3.1 The GTR Tracking Algorithm . . . . . . . . . 52

3.3.2 The HTF Tracking Algorithm . . . . . . . . 55

3.3.3 Final Track Candidates . . . . . . . . . . 56

3.4 Vertex Reconstruction ............... 57

3.5 Jet Reconstruction .................. 57

3.6 Electron Reconstruction and Identification . . . . . . . . . 59

3.6.1 EM Candidates ................. 59

3.6.2 Standard Electron Identification . . . . . . . . . 62

3.6.3 Electron Track Matching . . . . . . . . . 65

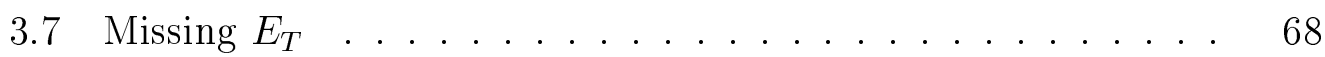

3.8 Muon Reconstruction .................. 69 
4 Data Sample, Event Generators, and Detector Simulations $\quad 71$

4.1 Data Sample................... 71

4.1.1 Online Triggering .............. 72

4.1.2 Trigger Efficiency ............... 73

4.2 Monte Carlo Simulation . . . . . . . . . . . . 75

4.2.1 Event Generation ............. 78

4.2.2 Detector Simulation . . . . . . . . . . 79

4.2.3 Corrections to the Monte Carlo Simulations . . . . . 80

5 Data Analysis and Background Studies $\quad 82$

5.1 Offline Event Selection . . . . . . . . . . . 82

5.1 .1 Electron ID Cuts ................ 83

5.1.2 Track Matching Requirement .......... 84

5.1.3 Central Di-Electron Pair . . . . . . . . . . 84

5.1.4 Like-Sign Di-Electron Pair . . . . . . . . . 87

5.1 .5 Invariant Mass Cut ............ 87

5.1.6 Missing Transverse Energy Cut . . . . . . . . 87

5.1 .7 The Candidate Event . . . . . . . . . . 90

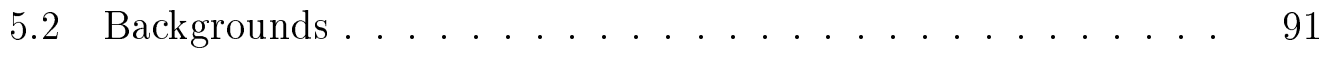

5.2.1 SM Background . . . . . . . . . . . . 94

5.2 .2 Instrumental background . . . . . . . . . . 95

5.2.3 Estimate of Uncertainties . . . . . . . . 100

5.3 Comparison Between the Data Sample and Backgrounds . . . 102

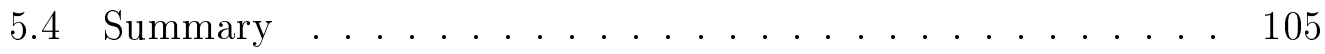


6 Results and Conclusion 107

6.1 Simulation of Signal . . . . . . . . . . . 107

6.2 Signal Efficiency . . . . . . . . . . . . . 109

6.3 Extraction of Cross Section Limits . . . . . . . . . 111

6.4 Cross Section Limits . . . . . . . . . . . . . 115

6.5 Future Improvement . . . . . . . . . . . . . . 118

$\begin{array}{ll}\text { A Electron Identification Efficiency } & \mathbf{1 1 9}\end{array}$

A.1 Loose Electron ID Efficiency . . . . . . . . . . . . . 119

A.2 Track Matching Efficiency . . . . . . . . . . . 122 


\section{List of Figures}

1.1 $\tilde{\chi}_{1}^{ \pm} \tilde{\chi}_{2}^{0}$ are pair produced at Tevatron via the $s$-channel and $t$ channel. ........................ 10

1.2 The three body decay of $\tilde{\chi}_{1}^{ \pm}$and $\tilde{\chi}_{2}^{0}$ via either virtual $\mathrm{W} / \mathrm{Z}$ bosons or sleptons $\tilde{l}^{ \pm} \ldots \ldots \ldots \ldots 11$

1.3 The distributions of $E_{T}$ for the leading leptons (top left), secondary leptons (top right), and the least energetic leptons (bottom left) in $\tilde{\chi}_{1}^{ \pm} \tilde{\chi}_{2}^{0}$ three-body decay. . . . . . . . . . . .

2.1 Fermilab accelerator chain. . . . . . . . . . . 16

2.2 Two-dimensional view of the DØdetector . . . . . . . . 22

$2.3 r-z$ view of the central tracking system consisting of the solenoid, SMT and CFT. . . . . . . . . . . . 24

2.4 The schematic of a CFT trigger channel, showing the VLPC, SIFT chip and the SVX II. . . . . . . . . . . . 27

2.5 Position resolution for CFT fiber doublet from cosmic ray test. 29

2.6 The expected $p_{T}$ resolution as a function of $|\eta|$ for the DØcentral tracking system.......................... 31

2.7 End view and side view of the CPS detector. . . . . . . . . 32 
2.8 Side view of the FPS detector. . . . . . . . . . . 34

2.9 Cutaway view of the DØcalorimeter. . . . . . . . . 37

2.10 The schematic view of a calorimeter cell consisting of the absorber plates and the signal board. . . . . . . . . 38

2.11 Side view of one quarter of the DØcalorimeter showing the "pseudo-projective" segmentation pattern. . . . . . . . 40

2.12 Schematic of the calorimeter readout electronics . . . . . . 42

3.1 The structure of the level 1 and level 2 triggers. The horizontal arrows denote information flow. . . . . . . . . . . 49

3.2 Level 3 framework. Data flow is indicated by the direction of

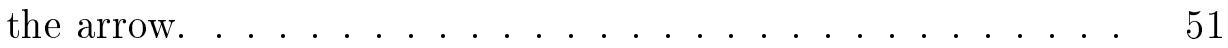

3.3 The distribution of EM fraction for electrons in $Z \rightarrow e e$ events and multijet events. The solid line is for $Z \rightarrow e e$ events and the

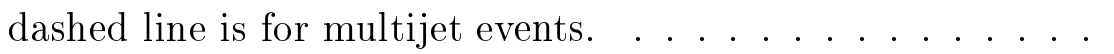

3.4 The distribution of isolation fraction for electrons in $Z \rightarrow e e$ events and multijet events. The solid line is for $Z \rightarrow e e$ events and the dashed line is for multijet events. . . . . . . . 64

3.5 The distribution of HMatrix $\chi^{2}$ for electrons in $Z \rightarrow e e$ events and multijet events. The solid line is for $Z \rightarrow e e$ and the dashed line is for multijet events. . . . . . . . . . . .

3.6 The $\chi^{2}$ probability distributions in the Run II Z data sample. The $\chi^{2}$ probability must be greater than 0.01 in order to establish a match between a loose electron and a track. . . . . . 
4.1 Delivered and recorded luminosity at DØin RunII during the year 2002 and 2003. . . . . . . . . . . . . . 72

4.2 Ratio between the number of low mass Drell-Yan events in data and in $\mathrm{MC}$ as a function of the primary electron $E_{T}$. . . 76

4.3 Ratio between the number of low mass Drell-Yan events in data and in $\mathrm{MC}$ as a function of the secondary electron $E_{T} \ldots \ldots 77$

5.1 $E_{T}$ distributions of the primary electrons in signal and background. The arrows represent the chosen cut. Number of events is normalized to an integrated luminosity of $124.5 \mathrm{pb}^{-1}$. . . 85

$5.2 E_{T}$ distributions of the secondary electrons in signal and background. The arrows represent the chosen cut. Number of events is normalized to an integrated luminosity of $124.5 \mathrm{pb}^{-1} \ldots$. .

5.3 Invariant mass distribution for like-sign electron pairs in the signal and backgrounds. The arrows represent the selection: 20 $\mathrm{GeV} / \mathrm{c}^{2}<M_{e e}<75 \mathrm{GeV} / \mathrm{c}^{2} \ldots \ldots \ldots . \ldots . \ldots 8$

5.4 Transverse missing energy distribution for the signal and backgrounds. The arrow represents the selection: $\mathbb{E}_{T}>15 \mathrm{GeV}$. The number of events is normalized to an integrated luminosity of $124.5 \mathrm{pb}^{-1} \ldots \ldots \ldots \ldots \ldots \ldots \ldots$

$5.5 \mathrm{r}-\mathrm{z}$ (top) and r- $\phi$ (bottom) view of the candidate event . . . . 92

5.6 Invariant mass distributions for QCD events which pass $\chi^{2}>$ 25 cut (top curve) and $\chi^{2}<20$ cut (bottom solid histogram). There is only 1 EM object with a track match in these events. 
5.7 distribution of fake probability of track matching for EM like object as a function of detector $\eta \ldots \ldots \ldots \ldots$

5.8 Invariant mass distributions for central di-electron sample (with 2 central tight electrons). Data (in dots with error bars) and sum of backgrounds (solid line) are consistent. . . . . . . . . 103

5.9 Missing $E_{T}$ distributions for central di-electron sample (with 2 central tight electrons). Data (in dots with error bars) and sum of backgrounds (solid line) are consistent. . . . . . . . . . . 104

6.1 Detection efficiency for $\tilde{\chi}_{1}^{ \pm} \tilde{\chi}_{2}^{0}$ pair as a function of chargino mass.111

6.2 The solid curve is the $95 \%$ C.L. upper limit of the $\tilde{\chi}_{1}^{ \pm} \tilde{\chi}_{2}^{0}$ production cross section times branching ratio to tri-electron. The hatched area up to $103.5 \mathrm{GeV} / \mathrm{c}^{2}$ was excluded by the LEP chargino searches. . . . . . . . . . . . . . 116

A.1 Illustration of sample sets used to measure the EMID efficiency. 120

A.2 The $2(\mathrm{tt})+(\mathrm{tp})$ (left) and $2(\mathrm{tt})+(\mathrm{tp})+(\mathrm{tf})$ (right) distributions for events with $2 \mathrm{CC}$ electrons. The points are data and the lines are the expected non- $Z$ background. . . . . . . . . . . 122

A.3 The $N_{1}+2 N_{2}$ (left) and $N_{0}+N_{1}+N_{2}$ (right) distributions for events with $2 \mathrm{CC}$ electrons in data. The points are data and the lines represent background. . . . . . . . . . . . . . 123 


\section{List of Tables}

1.1 Quarks and leptons, divided into 3 generations, in the Standard Model. ........................ 2

1.2 Mediators in the Standard Model. . . . . . . . . . . . 2

1.3 The matter field content of the MSSM. . . . . . . . . 6

1.4 The gauge and Higgs field content of the MSSM. . . . . . . 7

4.1 Single and Di-EM triggers used in this analysis. The details of each trigger term are explained in the text. . . . . . . 74

5.1 A summary of the successive cuts used in this analysis and the number of events passing each cut. . . . . . . . . 90

5.2 The candidate event summary with run number 169917 and event number $7189585 . \ldots \ldots \ldots \ldots$

5.3 The cross sections and number of MC events generated for the SM backgrounds. . . . . . . . . . . . . . 96

5.4 The statistical and systematic uncertainties for the estimate of backgrounds. ..................... 101 
5.5 The number of events passing the cumulative cuts listed in the first column for the backgrounds and data sample (last column). The comparison between data and backgrounds is described in

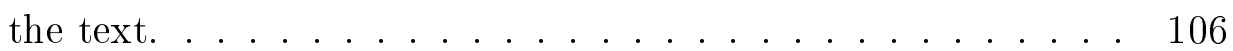

6.1 Chargino and Neutralino's masses and branching ratios for the five mSUGRA points. The remaining mSUGRA parameters are $m_{0}=100 \mathrm{GeV} / \mathrm{c}^{2}, \tan \beta=2.5, A_{0}=0$, and $\operatorname{sign}(\mu)=-1 \ldots \ldots$

6.2 Chargino masses, signal detection efficiencies, and the number of expected events in $124.5 \mathrm{pb}^{-1}$ for the five mSUGRA points.

6.3 Cross section times Branching ratio upper limit the 5 mSUGRA

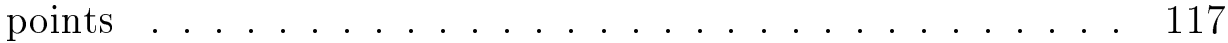




\section{Acknowledgements}

Doing research at Fermilab's DØ experiment is one of the most valuable experiences in my life. It involved many people from both Stony Brook and Fermilab. I would like to thank many of them who provided tremendous help to me during the process.

First of all, I would like to express my deepest thanks to my advisor, Prof. Michael Rijssenbeek, for many years' careful guidance to my graduate studies. The finish of the dissertation would not be possible without Michael's support and help. Particularly, I will never forget Michael's tremendous help, both mentally and financially, during the time I was stuck in China for 7 months with visa problems. It was Michael that encouraged me to start writing some parts of the dissertation during that time, which proved to be extremely useful later.

I would like to thank other members of the Stony Brook HEP group too. Prof. Paul Grannis helped me get the visa back to the U.S. when I thought nobody could help. Prof. John Hobbs helped me with my analysis and guided my first analysis note. Dr. Yuan Hu provided a lot of valuable discussions whenever I have questions on my analysis. He showed me that any questions could be answered eventually after careful thinking. Dr. Wendy Taylor tire- 
lessly revised my C.V. many times during my job-hunting. My officemate, Satish, has helped me in many ways he can.

I would like to thank many people from the DØ New Phenomenon group. Among them are the previous conveners: Gustaaf Brooijmans and Greg Landsberg. They reviewed my analysis results and gave many constructive suggestions. Also are the current NP group conveners Volker Buescher and JeanFrancois Grivaz. I feel very luck to get help from Volker, first as the convener of the EM id group then of the NP group. I thank Ulla for sharing some of her analysis results with me.

I would like to thank Prof. Jack Smith, Prof. Barbara Jacak and Dr. Serban Protopopescu for serving on both my Ph.D. candidate oral exam and dissertation defense, which are the two most important milestones in one's Ph.D. studies.

Life at Fermilab became very interesting when many Chinese students moved here. Among them, I would like to thank Jundong Huang for discussions on database and Junjie Zhu as an expert on EM id.

Finally, I would like to thank my family members. I thank my parents for raising me in such a happy family and teaching me the value of hardworking and striving for excellence; my elder brother, George, for doing many things first for me; and my wife, Xin, for giving me another happy family. 


\section{Chapter 1}

\section{Introduction}

\subsection{The Standard Model}

The Standard Model (SM) [1] is a Quantum Field Theory based upon the idea of local gauge invariance. The gauge symmetry group of the SM is $S U(3)_{C} \times S U(2)_{L} \times U(1)_{Y}$.

The particle content of the SM may be broadly classified in terms of two groups, namely the fundamental fermions (spin 1/2) and gauge vector bosons (spin 1). The fermions are further divided into two classes of particles called quarks and leptons. There are six flavors of quarks and of leptons, grouped pairwise into 3 generations as shown in Table 1.1. The six quarks are up $(u)$, down $(d)$, charm $(c)$, strange $(s), \operatorname{top}(t)$ and bottom $(b)$ quarks, and each quark flavor may exist in one of three possible color states. The six leptons are the electron $(e), \operatorname{muon}(\mu), \operatorname{tau}(\tau)$, and their corresponding neutrinos.

The forces between quarks and leptons are mediated by gauge vector bosons, as their properties listed in Table 1.2. The strong sector of the Stan- 


\begin{tabular}{|c||c|c|c|}
\hline \hline Generation & I & II & III \\
\hline \hline \multirow{2}{*}{ Quarks } & $\operatorname{up}(u)$ & $\operatorname{charm}(c)$ & $\operatorname{top}(t)$ \\
& $\operatorname{down}(d)$ & $\operatorname{strange}(s)$ & $\operatorname{bottom}(b)$ \\
\hline Leptons & electron $(e)$ & $\operatorname{muon}(\mu)$ & $\operatorname{tau}(\tau)$ \\
& electron neutrino $\left(\nu_{e}\right)$ & muon neutrino $\left(\nu_{\mu}\right)$ & tau neutrino $\left(\tau_{\nu}\right)$ \\
\hline \hline
\end{tabular}

Table 1.1: Quarks and leptons, divided into 3 generations, in the Standard Model.

dard Model is referred to as Quantum Chromodynamics (QCD), in which the strong force is described in terms of the gauge particles of $S U(3)_{C}$. These gauge vector bosons are $3^{2}-1=8$ in number and are named gluons $(g)$. Because the $S U(3)_{C}$ symmetry of the color interaction is believed to be exact, the gluons are massless. However, the non-abelian nature of the color symmetry allows for these gluons to interact among themselves as well as mediate the strong force between quarks.

\begin{tabular}{|c|c|c|c|}
\hline \hline Mediator & Symbol & Force Carried & Mass $\left(\mathrm{GeV} / c^{2}\right)$ \\
\hline \hline gluon & $g$ & strong & 0 \\
\hline $\mathrm{W} / \mathrm{Z}$ Bosons and Photon & $\mathrm{W}^{ \pm} / \mathrm{Z}^{0} / \gamma$ & electroweak & $80 / 91 / 0$ \\
\hline \hline
\end{tabular}

Table 1.2: Mediators in the Standard Model.

The electroweak sector of the Standard Model is a gauge theory based on 
$S U(2)_{L} \times U(1)_{Y}$ that unifies the weak and electromagnetic interactions. This symmetry requires 4 massless gauge vector bosons, but it is known that the $\mathrm{W}$ and $\mathrm{Z}$ bosons are massive $\left(80 \mathrm{GeV} / \mathrm{c}^{2}\right.$ and $91 \mathrm{GeV} / \mathrm{c}^{2}$ each). In order to provide mass to the $\mathrm{W}$ and $\mathrm{Z}$ bosons, the Higgs mechanism was introduced to break the symmetry spontaneously. However, the introduction of the Higgs mechanism requires a new particle, the spin-0 Higgs boson, to be added into the Standard Model. To date, there is no direct experimental evidence for the existence of the Higgs boson.

\subsection{The Problems of the Standard Model}

Although the Standard Model explains almost all the available data in current high energy experiment, it is considered to be incomplete due to various shortfalls. For example, the Higgs mechanism suffers from the "finetuning" problem. In any quantum field theory involving interacting fundamental scalars, the radiative correction to the scalar mass $\left(\delta m_{H}\right)$ diverges quadratically as the internal momentum in the loop increases. In lowest order in perturbation theory, the scalar mass is given by:

$$
m_{H}^{2}=m_{0}^{2}+\delta m_{H}^{2} \sim m_{0}^{2}-g^{2} \Lambda^{2}
$$

where $m_{0}$ is the bare Higgs mass parameter, $g$ is a dimensionless coupling constant of $\mathcal{O}(1)$, and $\Lambda$ is a cutoff scale where new physics or new particles becomes important. For the Standard Model, this scale is expected to be either the Grand Unification (GUT) scale $\Lambda \sim \mathcal{O}\left(10^{15}\right) \mathrm{GeV}$ or the Planck 
scale $\Lambda \sim \mathcal{O}\left(10^{19}\right) \mathrm{GeV}$. Following perturbative unitarity arguments [2], it is believed that $m_{H}$ can not be larger than a few hundred GeV. Equation 1.1 then states that if $\Lambda$ is in fact as large as the Planck scale or even the GUT scale, and if the coupling constant $g$ is of order unity, the two terms on the right hand side of the equation, each of which is $\sim 10^{30} \mathrm{GeV}^{2}$, have to combine to yield an answer $\leq 10^{6} \mathrm{GeV}^{2}$. While this possibility can not be logically excluded, it is a very unpleasant feature of the Standard Model. The problem is further complicated by the fact that the fine-tuning must be performed for each order in perturbation theory. Therefore, a method of controlling this divergence is preferred above this fine-tuning of parameters.

Yet another shortfall of the Standard Model is known as the "hierarchy problem" which is caused by the large unexplained difference between the electroweak and the Planck scales. In addition, the spontaneous breaking of the electroweak symmetry by the Higgs mechanism is introduced rather artificially and the Standard Model contains no hint of the origin of this breaking.

Theorists therefore have came up with various solutions to the above problems. One of the solutions is to make the scalars composite states of some more fundamental fermions. This is the approach of used in technicolor theories [3]. Another approach is to introduce a new symmetry that imposes the cancellation of the divergences without fine-tuning. Supersymmetry (SUSY) is such a symmetry. 


\subsection{Supersymmetry}

Supersymmetry $[4,5]$ is a symmetry between fermions and bosons. It solves the "fine-tuning" problem in an elegant way by postulating a SUSY partner (sparticle) for each Standard Model particle, with spin differing by $1 / 2$ and all other quantum numbers remaining the same. The introduction of SUSY particles cancels the quadratic divergence of the radiative corrections because of the sign difference in the contribution of fermion versus boson loop and the cancellation will be exact if SUSY is an exact symmetry. Although we know that SUSY is a broken symmetry since no selectron with mass of $0.511 \mathrm{MeV} / \mathrm{c}^{2}$ or smuon with mass of $0.106 \mathrm{GeV} / \mathrm{c}^{2}$ has been found, this does not re-introduce quadratic divergence. But if SUSY is to cure the fine-tuning problem, SUSY particles must be lighter than $\sim \mathcal{O}(1) \mathrm{TeV}$.

\subsubsection{Minimal Supersymmetric Standard Model}

The Minimal Supersymmetric Standard Model (MSSM) is the simplest SUSY extension of the Standard Model in which the smallest number of new particles and interactions are added. The MSSM adds to the Standard Model 1 additional Higgs field and the SUSY partners of all the Standard Model particles. With extra SUSY parameters defined, the production and decay of various sparticles are calculable through standard perturbation theory.

The matter field content of the MSSM is shown in Table 1.3. For each of the SM chiral fermions $f_{i}(\mathrm{i}=\mathrm{L}, \mathrm{R})$, it contains a spin zero sparticle $\tilde{f}_{i}$. Table 1.4 shows the gauge and Higgs field content of the MSSM. In MSSM, two 


\begin{tabular}{|c|c|c|c|}
\hline \hline Standard Model Particles & Symbol & Sparticles & Symbol \\
\hline quark & $q$ & squark & $\tilde{q}_{L, R}$ \\
\hline lepton & $l$ & slepton & $\tilde{l}_{L, R}$ \\
\hline \hline
\end{tabular}

Table 1.3: The matter field content of the MSSM.

Higgs doublets $h$ and $h^{\prime}$ are needed in order to give masses to all the fermions and avoid the gauge anomalies associated with fermion triangle diagrams [10]. These two Higgs doublets, containing 2 complex elements each, correspond to 8 degree of freedoms. Three of them give mass to the $\mathrm{W}$ and $\mathrm{Z}$ bosons and the rest corresponds to five Higgs particles: two charged scalar Higgs $H^{ \pm}$, two neutral scalar Higgs $H$ and $h$, and one neutral pseudoscalar Higgs A. The two vacuum expectation values (V.E.V.) of the higgs doublets are $\left\langle v_{1}\right\rangle$ and $\left\langle v_{2}\right\rangle$. The ratio of the $\left\langle v_{2}\right\rangle /\left\langle v_{1}\right\rangle$ is referred to as $\tan \beta$, a parameter which plays an important role in MSSM. Another MSSM parameter originating from the Higgs sector is the Higgsino mass parameter $\mu$. The electroweak gauginos (Winos and Binos) and Higgsinos mix to form mass eigenstates. Bino, neutral Winos, and neutral Higgsinos mix to form four neutralino's $\left(\tilde{\chi}_{1,2,3,4}^{0}\right)$ with $\left(m_{\tilde{\chi}_{1}^{0}}<m_{\tilde{\chi}_{2}^{0}}<m_{\tilde{\chi}_{3}^{0}}<m_{\tilde{\chi}_{4}^{0}}\right)$. Similarly, charged Winos and charged Higgsinos form two chargino's $\left(\tilde{\chi}_{1,2}^{ \pm}\right)$with $\left(m_{\tilde{\chi}_{1}^{ \pm}}<m_{\tilde{\chi}_{2}^{ \pm}}\right)$. The SUSY partners of the gluons are called gluinos. 


\begin{tabular}{|c|c|c|c|}
\hline \hline Particles & Symbol & Sparticles & Symbol \\
\hline gluon & $g$ & gluino & $\tilde{g}$ \\
\hline charged Higgs & $H^{ \pm}$ & & \\
\hline charged weak boson & $W^{ \pm}$ & chargino & $\tilde{\chi}_{1,2}^{ \pm}$ \\
\hline $\begin{array}{c}\text { light Higgs } \\
\text { heavy Higgs }\end{array}$ & $H$ & & \\
pseudoscalar Higgs & $A$ & neutralino & $\tilde{\chi}_{1,2,3,4}^{0}$ \\
neutral weak boson & $Z$ & & \\
photon & $\gamma$ & & \\
\hline \hline
\end{tabular}

Table 1.4: The gauge and Higgs field content of the MSSM.

\subsubsection{R-parity and LSP}

A new multiplicative quantum number, $\mathcal{R}$, is introduced in SUSY models. $\mathcal{R}$ is defined as:

$$
\mathcal{R}=(-1)^{3 B+L+2 S}
$$

where $B$ is the baryon number, $L$ is the lepton number, and $S$ is the spin of the particle: $\mathcal{R}=+1$ for SM particles and $\mathcal{R}=-1$ for SUSY particles. The total $\mathcal{R}$ of a state is calculated by multiplying all of the individual particles' and sparticles' $\mathcal{R}$ 's. SUSY models can be classified as $\mathcal{R}$-parity conserving and $\mathcal{R}$-parity violating.

In all current colliders, the initial state is assumed to only contain SM particles, so $\mathcal{R}_{\text {initial }}=+1$. In our analysis, we assume the conservation of 
$\mathcal{R}$-parity, which has the following important implications:

- Sparticles can only be pair-produced in collisions of ordinary particles.

- The existence of the lightest SUSY Particle (LSP), which is relatively stable and assumed to be neutral and weakly interacting like neutrino's.

Therefore, a general SUSY interaction with $\mathcal{R}$-parity conservation at Tevatron can be described by:

$$
p \bar{p} \rightarrow 2 L S P+X
$$

where X represents collections of SM particles such as leptons or quarks. Due to the presence of the LSP in the final state, missing energy is usually presented in the SUSY events.

\subsubsection{Minimal Supergravity}

As mentioned above, SUSY is a broken symmetry. In order to break SUSY in the MSSM, one needs to add a large number (> 100) of soft SUSY breaking terms. Unless further theories about the SUSY breaking are in place, this large number of terms makes the SUSY theory unpredictive and intractable. One attractive theory is the Minimal Supergravity (mSUGRA) $[6,7]$ which unifies the strong, weak, electromagnetic and gravitational forces at some very large energy scale, $M_{X}$. This energy scale may be either the GUT scale or the Planck scale. Only 4 parameters and 1 sign are needed to describe the mSUGRA models: 
- $m_{0}$, the common scalar mass at the $M_{X}$ scale

- $m_{1 / 2}$, the common gaugino mass at the $M_{X}$ scale

- $\tan (\beta)$, the ratio of the vacuum expectation value of the two Higgs doublets

- $A_{0}$, common trilinear coupling constant at the $M_{X}$ scale.

- $\operatorname{sign}(\mu)$, the sign of Higgsino mass parameter.

Given the above mSUGRA parameters, one can determine the masses and mixing angles of SUSY particles at the electroweak scale by evolving the SUSY parameters from the unification scale down to the electroweak scale using the Renormalization Group Equations(RGEs). In mSUGRA, sparticle masses are dominantly functions of $m_{0}$ and $m_{1 / 2}$ and high values usually result in high sparticle mass. The following relationship between the mass of $\tilde{\chi}_{1}^{ \pm} / \tilde{\chi}_{1,2}^{0}$ and $m_{1 / 2}$ approximately holds over most of the mSUGRA parameter space:

$$
\begin{gathered}
m\left(\tilde{\chi}_{1}^{ \pm}\right) \approx m\left(\tilde{\chi}_{2}^{0}\right) \approx 0.8 \mathrm{~m}_{1 / 2} \\
m\left(\tilde{\chi}_{1}^{0}\right) \approx 0.4 \mathrm{~m}_{1 / 2}
\end{gathered}
$$

In mSUGRA, the LSP is the lightest neutralino $\left(\tilde{\chi}_{1}^{0}\right)$. 


\subsection{Search for $\tilde{\chi}_{1}^{ \pm} \tilde{\chi}_{2}^{0}$ in the Like-Sign Dilepton channel}

Our analysis searches for $\tilde{\chi}_{1}^{ \pm} \tilde{\chi}_{2}^{0}$ decaying into a final state containing a likesign electron pair and missing energy. We first discuss the production of the $\tilde{\chi}_{1}^{ \pm} \tilde{\chi}_{2}^{0}$ pair and the leptonic decay of $\tilde{\chi}_{1}^{ \pm}$and $\tilde{\chi}_{2}^{0}$ in the following.

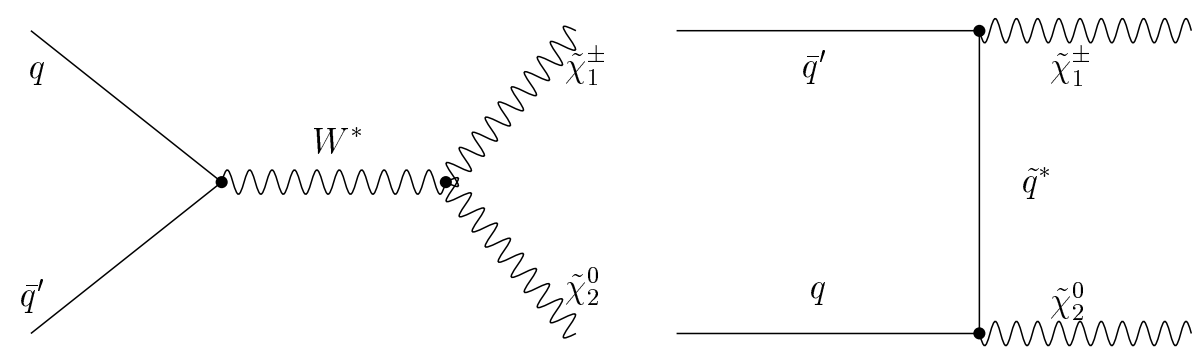

Figure 1.1: $\tilde{\chi}_{1}^{ \pm} \tilde{\chi}_{2}^{0}$ are pair produced at Tevatron via the $s$-channel and $t$ channel.

In mSUGRA, the sleptons $(\tilde{l})$, the lighter chargino $\left(\tilde{\chi}_{1}^{ \pm}\right)$and the lighter neutralinos $\left(\tilde{\chi}_{1}^{0}\right.$ and $\left.\tilde{\chi}_{2}^{0}\right)$ are typically less massive than gluinos and squarks. This causes the production of $\tilde{\chi}_{1}^{ \pm} \tilde{\chi}_{2}^{0}$ pairs to have the largest cross section and dominate sparticle production over much of the mSUGRA parameter space. Figure 1.1 shows the associated $\tilde{\chi}_{1}^{ \pm} \tilde{\chi}_{2}^{0}$ production via the $s$-channel and $t$ channel at Tevatron. Quark and antiquark annihilate to produce $\tilde{\chi}_{1}^{ \pm}$and $\tilde{\chi}_{2}^{0}$ in the $s$-channel through a virtual W boson $\left(q \bar{q}^{\prime} \rightarrow W^{ \pm *} \rightarrow \tilde{\chi}_{1}^{ \pm} \tilde{\chi}_{2}^{0}\right)$, and in the t-channel through virtual squark $(\tilde{q})$ exchange. In most cases, the contribution from the $t$-channel to the $\tilde{\chi}_{1}^{ \pm} \tilde{\chi}_{2}^{0}$ is suppressed by the large mass of the squarks. 

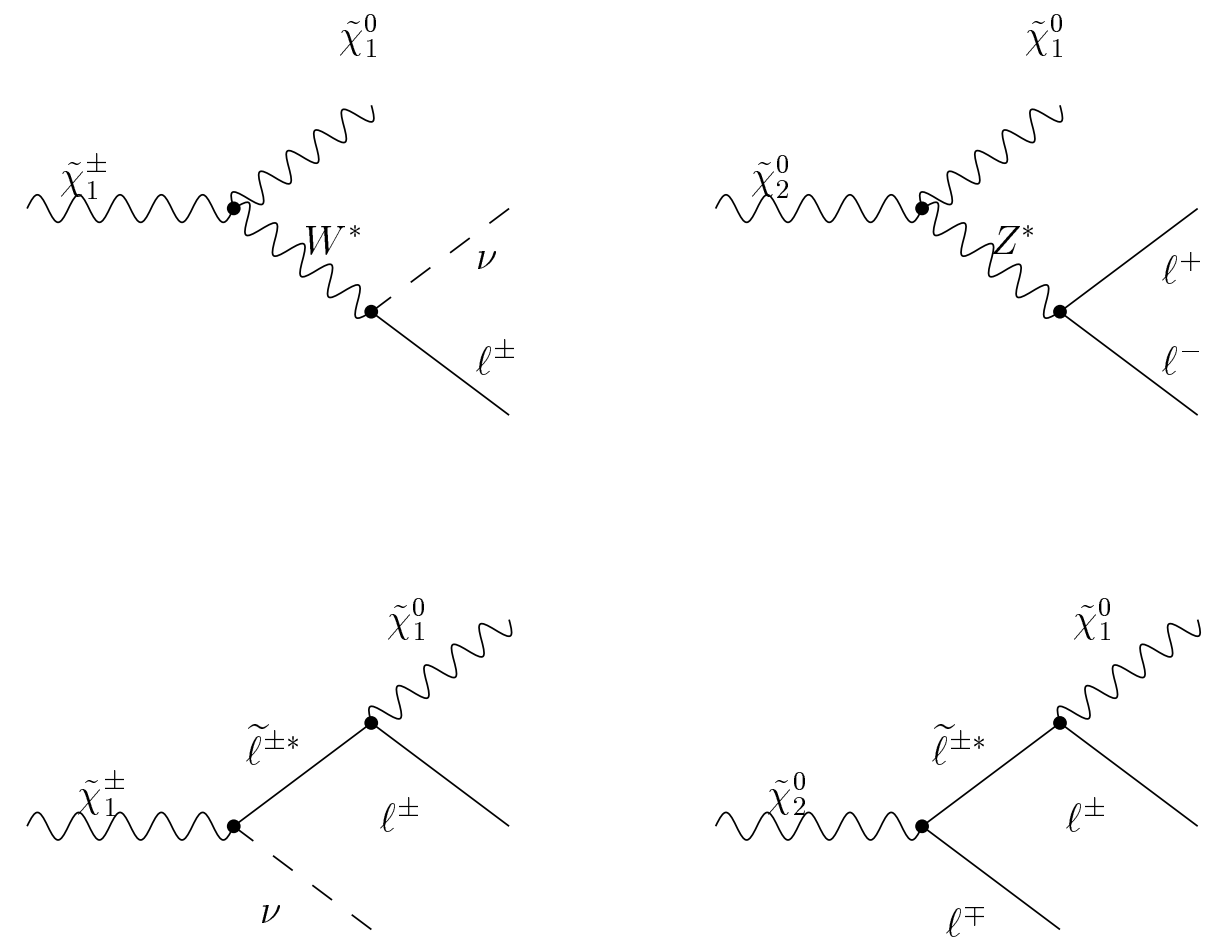

Figure 1.2: The three body decay of $\tilde{\chi}_{1}^{ \pm}$and $\tilde{\chi}_{2}^{0}$ via either virtual $\mathrm{W} / \mathrm{Z}$ bosons or sleptons $\tilde{l}^{ \pm}$. 
$\tilde{\chi}_{1}^{ \pm}$can decay into $q \bar{q}^{\prime}$ or $l \bar{\nu}_{l}$ plus an LSP, while the neutralino can decay into $q \bar{q}$ or $l \bar{l}$ plus an LSP. When both $\tilde{\chi}_{1}^{ \pm}$and $\tilde{\chi}_{2}^{0}$ decay leptonically, they generate a final state consisting of 3 isolated leptons plus missing energy. Figure 1.2 shows the three-body leptonic decays of $\tilde{\chi}_{1}^{ \pm} \tilde{\chi}_{2}^{0}$ via either virtual $\mathrm{W} / \mathrm{Z}$ bosons or virtual sleptons. Since this signature has a very low background from Standard Model processes, it has long been suggested as one of the most promising channels for the discovery of SUSY at Tevatron [8].

The leptons generated in the three-body decay are expected to have relatively low $E_{T}$. Figure 1.3 shows the $E_{T}$ distribution of the final state leptons from a Monte Carlo sample where the $\tilde{\chi}_{1}^{ \pm}$mass is $86.9 \mathrm{GeV} / \mathrm{c}^{2}$. It is obvious that the least energetic lepton is very soft and low in $E_{T}$, which will make it difficult to detect.

The Like-Sign Di-lepton (LSD) channel has been suggested [9] as a complement to the tri-lepton channel. In the LSD channel, we drop the requirement for the third lepton but require the two leptons to have the same charge. In this analysis, we apply the LSD search for the first time to the DØ data and focus in the like-sign di-electron channel. 

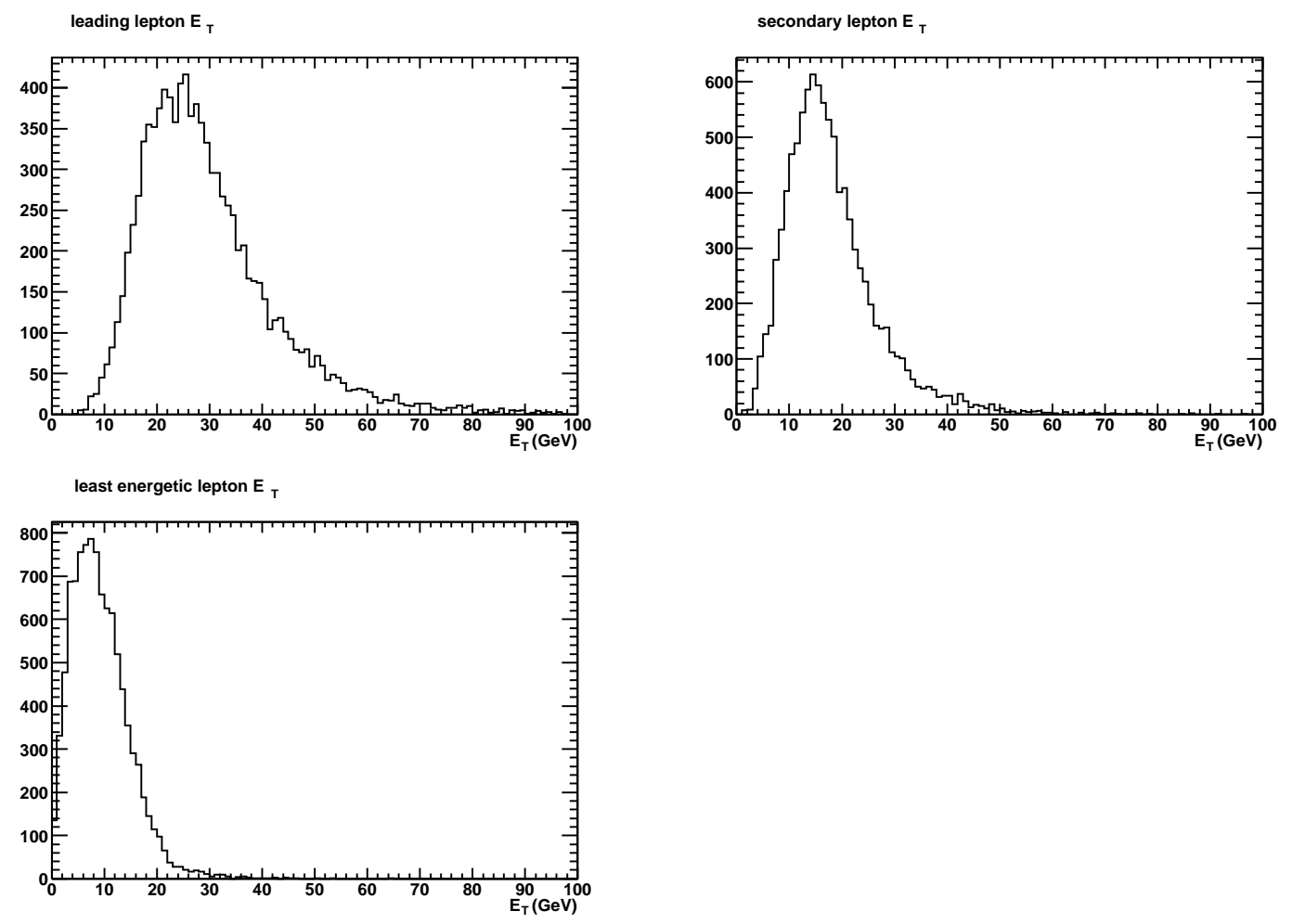

Figure 1.3: The distributions of $E_{T}$ for the leading leptons (top left), secondary leptons (top right), and the least energetic leptons (bottom left) in $\tilde{\chi}_{1}^{ \pm} \tilde{\chi}_{2}^{0}$ threebody decay. 


\section{Chapter 2}

\section{Experimental Apparatus}

The data used in this analysis was collected with the DØ detector located at the Fermilab Tevatron collider. This chapter provides a brief overview of the Fermilab accelerator complex and the technical details of the D $\varnothing$ detector, with emphasis on tracking and calorimetry.

\subsection{The Fermilab Accelerators}

Fermilab's Tevatron is currently the world's highest energy particle accelerator and collider. In Run I, the Tevatron operated at a center of mass energy of $1.8 \mathrm{TeV}$, a bunch crossing frequency of $300 \mathrm{kHz}$ (bunch spacing of $3.5 \mu \mathrm{s}$ ) and a typical instantaneous luminosity of $0.16 \times 10^{32} \mathrm{~cm}^{-2} \mathrm{~s}^{-1}$. In Run II, the upgraded Tevatron was increased in center of mass energy to $1.96 \mathrm{TeV}$ and the bunch spacing was reduced to $396 \mathrm{~ns}$. The instantaneous luminosity has also been increased and now reaches up to $5 \times 10^{31}$ on a regular basis, with a goal of $\sim 10^{32} \mathrm{~cm}^{-2} \mathrm{~s}^{-1}$. For the whole Run II period, the Tevatron will deliver at least 20 times more integrated luminosity than in Run I. 
Figure 2.1 shows the diagram of the Fermilab accelerator chain. The main components of the Fermilab accelerator chain are: $[11,12]$

- Cockcrofton-Walton Pre-Accelerator

- Linear Accelerator (The Linac)

- Booster synchrotron

- Main Injector

- Antiproton Source

- Debuncher and Accumulator

- Recycler

- Tevatron

In the following sections, we will shortly describe these components.

\subsubsection{Pre-Accelerator, LINAC, and the Booster}

The proton beam begins life as negative hydrogen ions $\left(\mathrm{H}^{-}\right)$from a magnetron surface-plasma source. These ions are then accelerated through a static electric field to $750 \mathrm{keV}$ by a commercial Cockcroft-Walton accelerator. Upon exiting the pre-accelerator, transport lines direct the $\mathrm{H}^{-}$ions into the 500foot-long linear accelerator (the Linac) where oscillating electric fields boost the negative $H^{-}$ions to the energy of $400 \mathrm{MeV}$. The ions subsequently pass 


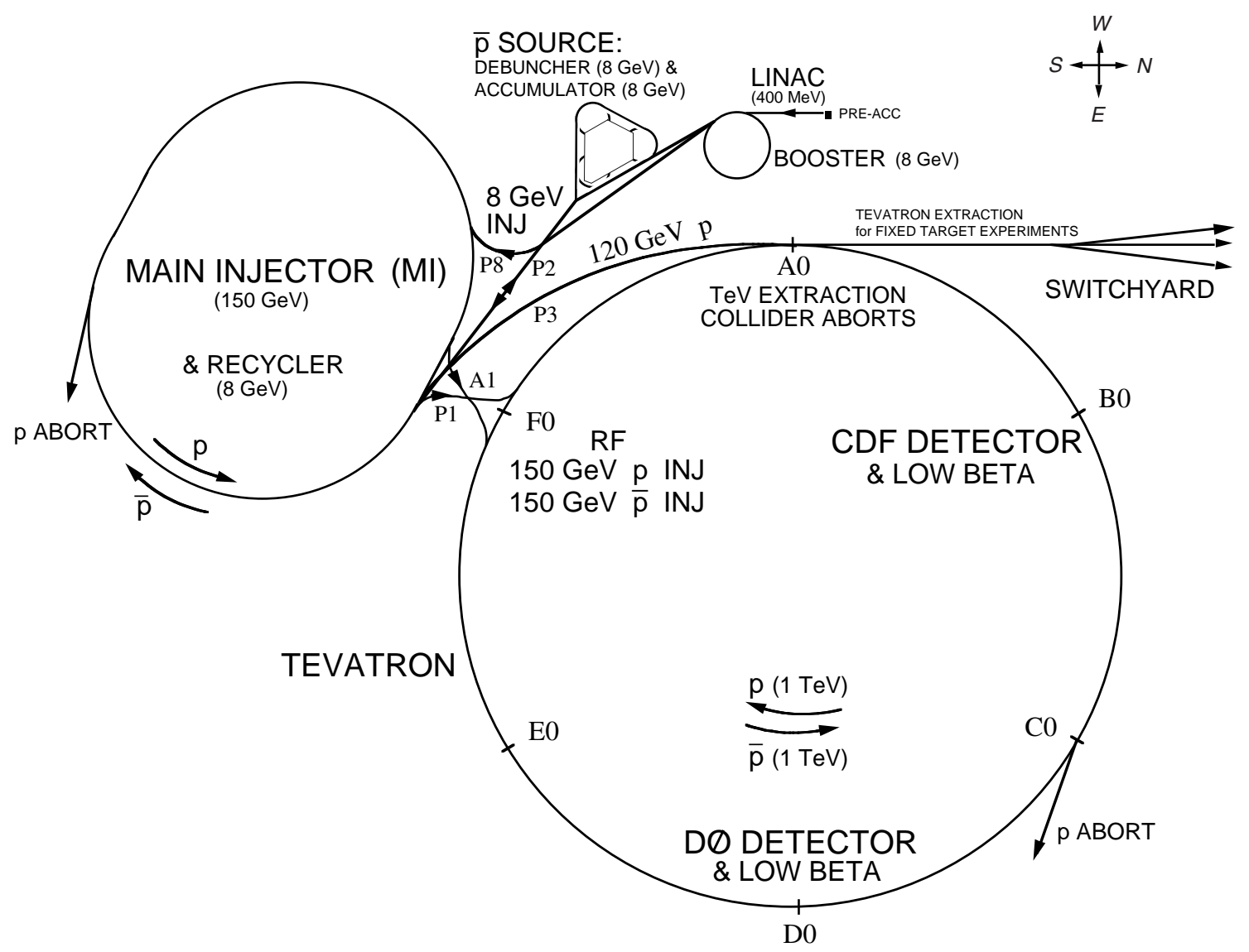

Figure 2.1: Fermilab accelerator chain. 
through a carbon foil, which strips both electrons from each $H^{-}$ion, leaving only the protons.

The next acceleration stage is a 1570-foot-circumference fast cycling synchrotron ring called the Booster. Within the booster, protons pass through a series of RF cavities spaced around the ring. As the protons pass through the cavities they accelerate. Magnetic fields produced by focusing and bending magnets act to keep the protons circulating in a stable orbit. As the RF frequencies are raised in sync with the accelerating protons, the currents in the magnets are also raised synchronously. The protons travel around the Booster about 20,000 times and reach an energy of $8 \mathrm{GeV}$. A pulse train of approximately $5-7$ bunches, each containing $5-6 \times 10^{10}$ protons are injected into the next acceleration stage, the Main Injector.

\subsubsection{The Main Injector and Antiproton Production}

The Main Injector (MI) [13] was built for Run II to replace the main ring in Run I. It is capable of delivering three times as many protons to the Tevatron as the old Main Ring. It is a $150 \mathrm{GeV}$ synchrotron with a circumference of about 2 miles. The MI coalesces proton bunches exiting the Booster into a single high intensity bunch of approximately $5 \times 10^{12}$ protons and accelerates protons to the energy of $150 \mathrm{GeV}$. The MI also has another very important function: generating anti-protons. When protons are accelerated to the energy of $120 \mathrm{GeV}$, they are extracted to impact on an external nickel/copper target to produce antiprotons. The antiprotons are then injected into the antiproton storage rings. 
The function of the antiproton storage rings is to squeeze the initially coherent antiproton beam into as compact a phase space as possible. The antiproton storage rings are comprised of the Debuncher and the Accumulator. At first, the antiprotons exhibit large variations in momentum. Many oscillate in directions transverse to the desired beam orbit. Within the Debuncher, a process known as stochastic cooling is used to reduce the momentum spread of the antiprotons and to reduce as much as possible their oscillations transverse to the designed beam orbit. This is done by applying correction signals to the particles to minimize their deviations from an ideal orbit. Once a small emittance beam is achieved, the $8 \mathrm{GeV}$ antiprotons are transferred to the Accumulator ring where they are further cooled stochastically. Here, stored antiproton bunches are produced with stacking rates about $10^{11}$ antiprotons per hour. After the accumulator, the density of the anti-protons is significantly increased. When the antiproton stack is large enough $\left(\sim 10^{12}\right)$, they are transferred to the $8 \mathrm{GeV}$ Recycler ring.

The Recycler is an $8 \mathrm{GeV}$ permanent magnet storage ring utilizing stochastic cooling system. It is capable of delivering more anti-protons to the Tevatron and therefore, proportionally increases the beam luminosity. The main function of the Recycler is to operate as a recovery channel for antiprotons left at the end of a previous collider store. The Recycler accumulates and re-cools dilute $1-6 \times 10^{11}$ anti-protons up to a total stack of about $3 \times 10^{12}$ antiprotons. Once the accumulated antiprotons reach the energy of $8 \mathrm{GeV}$, they are extracted to the the Main Injector and accelerated to the energy of $150 \mathrm{GeV}$. The $150 \mathrm{GeV}$ proton-antiproton bunches from the Main Injector are delivered 
in opposite directions into the Tevatron.

\subsubsection{The Tevatron}

The Tevatron [12] is a synchrotron with a diameter of about 2 kilometers. Superconducting magnets are cooled to a temperature of $4.8 \mathrm{k}$ and produce fields of 4 Tesla. Thirty-six bunches of protons and thirty-six bunches of antiprotons ("36 × 36" mode) are accelerated by RF from $150 \mathrm{GeV}$ to 0.96 $\mathrm{TeV}$. Once the desired final energy of $0.96 \mathrm{TeV}$ is reached for both the proton bunches and the antiproton bunches, the two beams are squeezed into small transverse cross sections through the use of quadrupole magnets and collide at two luminous regions: B0 $(\mathrm{CDF})$ and D0 (DØ). Proton and antiproton beam crossings take place about every 396 ns. The typical initial instantaneous luminosity of a store at present is around $0.5 \times 10^{32} \mathrm{~cm}^{-2} \mathrm{~s}^{-1}$. As beams interact, a store's luminosity will continually decrease from the initial value. The beam lifetime of a store is of the order of 20 hours and the time interval between stores is several hours.

\subsection{The DØ Coordinate System}

Before proceeding with a description of the D $\varnothing$ detector, it is necessary to define the coordinate system used in the experiment. D $\varnothing$ uses a right-handed coordinate system. The beam axis runs approximately from north to south at the $\mathrm{D} \varnothing$ interaction region. The $+z$ axis is defined along the proton beam direction and $-z$ axis is along the antiproton beam direction. The $x$ axis 
is horizontal with $+x$ pointing toward the Tevatron center and the $y$ axis is vertical with $+y$ pointing upward.

Azimuthal angles, $\phi$, are measured with respect to the $+x$ axis so that $\phi=0$ coincides with the $+x$ axis and $\phi=\frac{\pi}{2}$ with the $+y$ axis. The range of $\phi$ is from 0 to $2 \pi$. Polar angles, $\theta$, are measured from the $+z$ axis and are from 0 to $\pi$. In stead of using the polar angle $\theta$, we often use pseudorapidity $\eta$, which is defined as:

$$
\eta=-\log \left(\tan \frac{\theta}{2}\right)
$$

The pseudorapidity is an approximation of the rapidity $y$, which is defined as:

$$
\eta=\frac{1}{2} \ln \left(\frac{E+p_{z}}{E-p_{z}}\right)
$$

one can easily verify that $\eta \approx y$ when the mass of a particle is much smaller than its energy. Because the rapidity is invariant under Lorentz transformations, by using the pseudorapidity, the shape of the particle distribution $(d N / d \eta)$ is invariant under boosts along the z-axis. Usually, two types of pseudorapidity are used. One is the physical pseudorapidity $\left(\eta_{p h y}\right)$, which is calculated with respect to the primary vertex. The other one is the detector pseudorapidity $\left(\eta_{\text {det }}\right)$, which is calculated with respect to the detector's center at $z=0$.

Another quantity often used at $\mathrm{D} \varnothing$ is the transverse energy $E_{T}$ :

$$
E_{T}=E \sin \theta
$$

where $E$ is a particle's total energy. For massless or highly relativistic par- 
ticles, $E_{T}=p_{T}$ is a good approximation. The reasons for using transverse energy are two-fold. First, hard scattering interactions, those most likely to produce new particles, are characterized by interactions with high transverse momentum to the initial beam direction. Massive particles also give high $p_{T}$ decay products. Second, in $\mathrm{p} \overline{\mathrm{p}}$ collisions, many of the secondary particles may escape measurement by staying within the beam pipe which makes the application of conservation of momentum and energy in the $z$ direction impossible. However, as the transverse momenta of such forward particles are negligible, one can apply conservation of momentum in the transverse plane to infer the missing transverse energy $\not_{T}=\sqrt{E_{x}^{2}+E_{y}^{2}}$.

\subsection{The DØ Detector}

The D $\varnothing$ experiment uses a large general-purpose particle detector to study the $\mathrm{p} \overline{\mathrm{p}}$ collisions at the Tevatron Collider. One of the prime physics focus of the experiment is the study of phenomena involving high $p_{T}$ and high mass states such as the precision study of the top quark and $W / Z$ bosons, and the search for heavy exotic particles like supersymmetric particles. The DØ detector [15] has excellent ability to identify leptons and measure their energy, to measure the energy and direction of parton jets, and to determine the missing transverse energy. It was upgraded significantly from 1996 to 2001 in order to operate with the upgraded Tevatron which has a 396 ns beam crossing time and instantaneous luminosity up to $\sim 10^{32} \mathrm{~cm}^{-2} \mathrm{~s}^{-1}$. The upgrade builds on the strengths of DØ, full coverage in calorimetry and muon detection, 


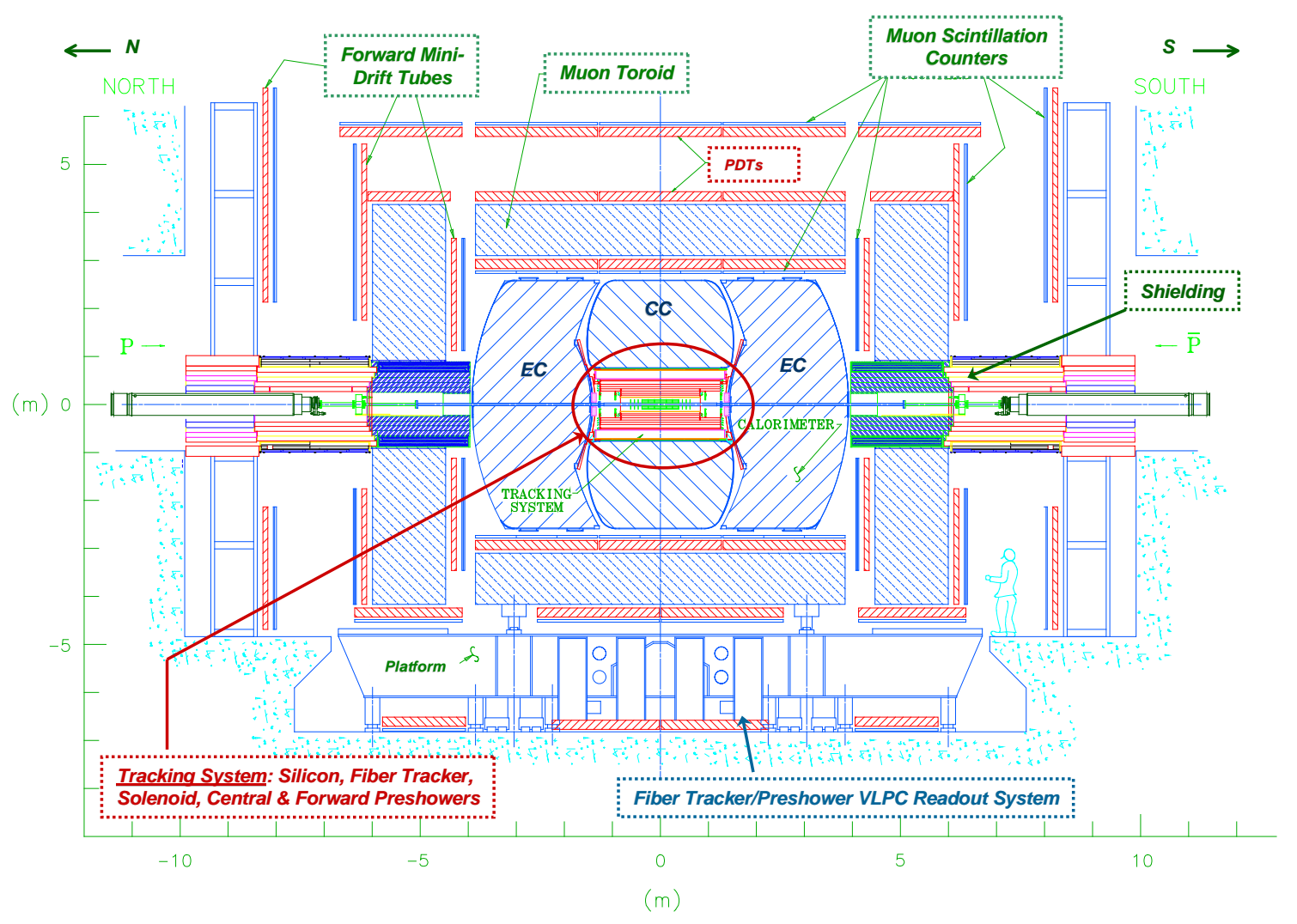

Figure 2.2: Two-dimensional view of the D $\varnothing$ detector 
while enhancing the tracking and triggering capabilities. The upgraded DØ detector [14] is shown in Figure 2.2. We will describe each of the sub-detector in some details in the following sections.

\subsubsection{Central Tracking System}

The DØ's new central tracking system, shown in Figure 2.3, consists of a 2 Tesla central solenoidal magnetic field, a silicon tracker close to the beam pipe and a fiber tracker surrounding it. The tracking system has been designed to obtain good momentum and vertex measurement.

\section{The Solenoid}

The new 2 Tesla superconducting solenoid magnet [16] added to the D $\varnothing$ detector was located in the bore of the central calorimeter cryostat. Thus, the charge and momentum of a charged particle can be determined from the curvature of its trajectory in the new central tracking system. The solenoid is a $2.7 \mathrm{~m}$ long two-layer coil with a mean radius of $60 \mathrm{~cm}$. The thickness of the coil plus its cryostat has been minimized in order to preserve good electromagnetic resolution (about 0.9 radiation lengths). The uniformity of the magnetic field inside the tracking volume is measured to be within $0.5 \%$.

\section{Silicon Microstrip Tracker}

The basic function of the DØ Silicon Microstrip Tracker (SMT) [17] is to reconstruct tracks and vertices over the full $\eta$ acceptance of DØ. Because 


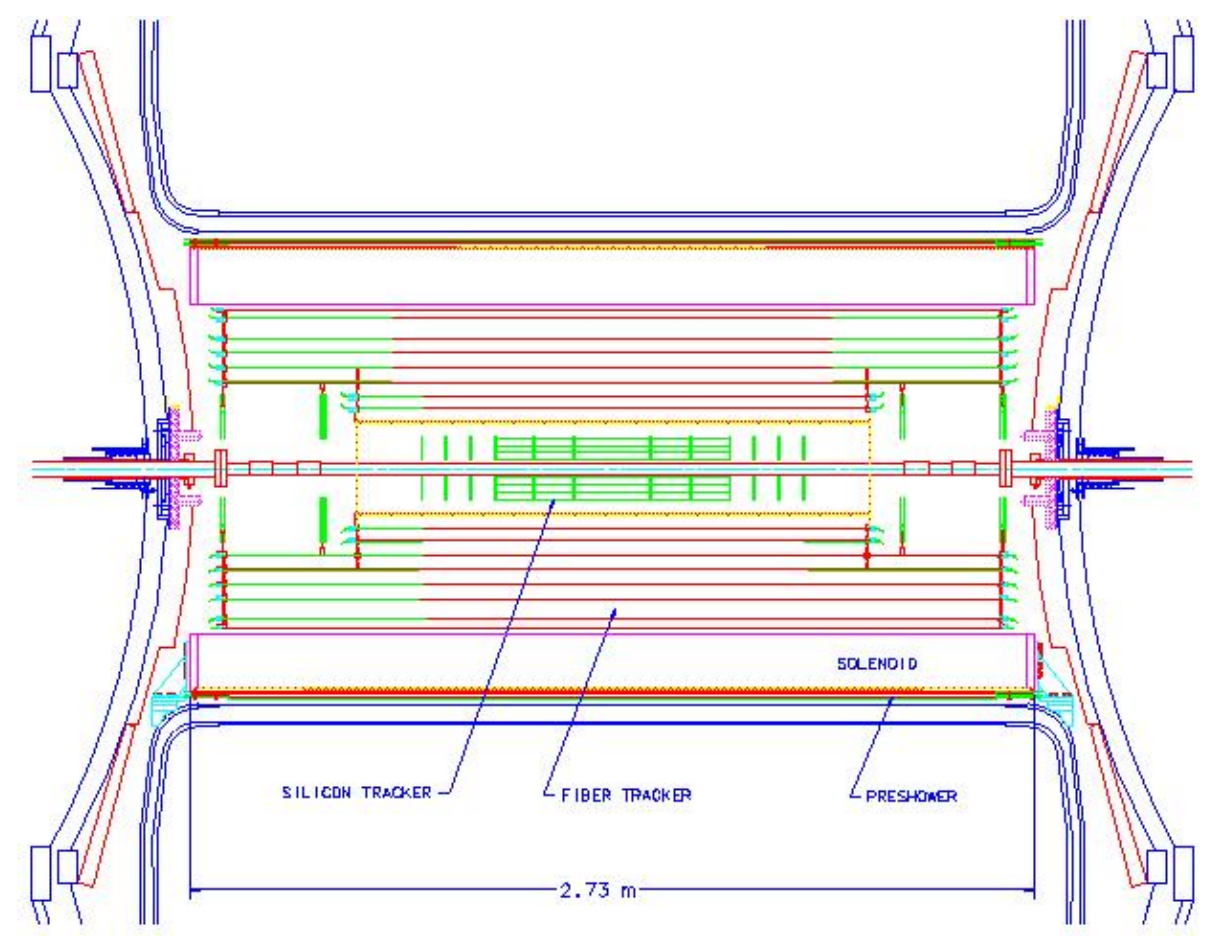

Figure 2.3: $r-z$ view of the central tracking system consisting of the solenoid, SMT and CFT. 
of the large spread of the luminous region in the $z$ direction, with $\sigma_{z}=25$ $\mathrm{cm}$, the SMT is designed as a hybrid system with barrel detectors measuring primarily the $r-\phi$ coordinate and disk detectors measuring the $r-z$ and $r-\phi$ coordinate.

The SMT detector shown in Figure 2.3 has 6 barrel segments, 12 small diameter "F" disks and 4 large diameter "H" disks. Each barrel segment is 12 $\mathrm{cm}$ long and has 4 detector layers.

Layer 1 and layer 3 are divided into the central and end region. Four central barrel segments are double-sided detectors with axial and $90^{\circ} z$ strips while two end barrel segments are single-sided detectors with axial strips only. Layer 2 and layer 4 of the barrel are not divided into central/end region and are double-sided detectors with axial and $2^{\circ}$ stereo strips. F disks are double-sided detectors with $\pm 15^{\circ}$ stereo angle. Four of the $\mathrm{F}$ disks are sandwiched in $8 \mathrm{~mm}$ gaps between barrel segments and the remaining 8 disks are symmetrically located on each side of the barrel. H disks are doubled-sided detectors as well, with a $\pm 7.5^{\circ}$ stereo angle. The four $\mathrm{H}$ disks are located at $|z|=110 \mathrm{~cm}$ and $120 \mathrm{~cm}$.

The SMT is built on small-pitch silicon wafers providing a spatial resolution of approximately $10 \mu \mathrm{m}$. The silicon wafers are mounted onto ladder and disk structures for the barrels and disks respectively. In each ladder, two 60 $\mathrm{mm}$ long silicon wafers are wire-bonded together, forming a $12 \mathrm{~cm}$ long unit mounted between two beryllium bulkheads. The ladders are grouped into 24 equal $\phi$ towers centered on layer 3 ladders. Each disk contains 12 wedgeshaped silicon wafers. The wedges alternate from one surface to the other of a 
beryllium dodecagon support structure. Disks are oriented so that the wedges match the barrel towers.

Charge deposited on each silicon strip is collected and digitized by SVX II chips. Each SVX II chip has 128 identical channels of analog-to-digital conversion and storage. The SVX II chips are connected to a flexible printed circuit called a High Density Interconnect (HDI). At the outer radius of the detector, The HDIs are connected by two metallic cables to readout cards (port cards) mounted in crates located on the platform. The port card controls the SVX II chips and converts data to optical signals. At rates approaching 1 Gbits/s, optical signals are sent through $150 \mathrm{ft}$ of multi-mode fiber optic cable to the data acquisition system.

\section{Central Fiber Tracker}

The Central Fiber Tracker (CFT) [18] shown in Figure 2.3, surrounds the silicon vertex detector and covers the central pseudorapidity region. The detector utilizes the basic principles of scintillation light production, collection, and detection. Charged particles produced in a p $\bar{p}$ collision that pass through the fiber volume will deposit energy by ionization, a portion of which is converted into scintillation light. A fraction of this light is optically trapped inside the fiber and travels to both ends of the fiber. At one end, the light encounters an aluminum mirror coating and is reflected back into the fiber. At the other end, the fiber is mated to a clear optical waveguide fiber which pipes the light to a high quantum efficiency photodetector.

At DØ, the CFT consists of 8 concentric carbon fiber cylinders occupying 
the radial space from 20 to $50 \mathrm{~cm}$. The cylinders are covered on both inside and outside by scintillating fibers of $835 \mu \mathrm{m}$ in diameter and $1.7-2.6 \mathrm{~m}$ in axial length. A fiber doublet layer with the fibers oriented in the axial direction is mounted on each of the eight cylinders. An additional doublet layer oriented in either the $u$ or $v$ stereo angle of approximately $2^{\circ}$ is mounted on successive cylinders. From the smallest radius outward, the orientations on the cylinders are $x u-x v-x u-x v-x u-x v-x u-x v$. There are about 77 thousand scintillating fibers in total in the CFT.

The photodetector used in the CFT is called Visible Light Photon Counter (VLPC) [20]. It is a silicon-avalanche device with the following characteristics: $70 \%$ quantum efficiency for the wavelength range of interest, gain of greater than 40,000 , and operating with a bias voltage of $6-8 \mathrm{~V}$ at a temperature of $7-10 \mathrm{~K}$.

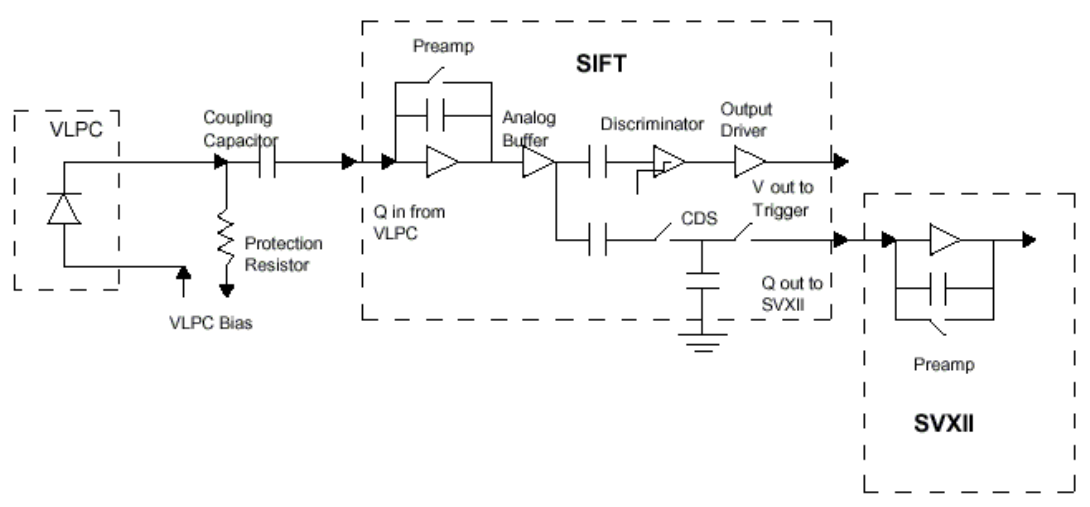

Figure 2.4: The schematic of a CFT trigger channel, showing the VLPC, SIFT chip and the SVX II. 
The total collection time of the CFT signals from an interaction is considerably shorter than the 396 ns bunch spacing. This makes the CFT an important element of the D $\varnothing$ trigger system (Section 3.1). To allow parallel processing, CFT is divided into 80 equal azimuthal sectors and hits from all 8 axial layers are used to look for hits-in-roads, i.e. hits that line up, to form CFT level 1 trigger tracks. Figure 2.4 shows the front-end electronics for a CFT trigger channel. To extract the CFT trigger signal, a SIFT chip is added between the VLPC output and SVX II chip. The SIFT chip amplifies and buffers the VLPC signal. In two different time slices, the SIFT chip discriminates each input signal and outputs a $3.3 \mathrm{~V}$ single ended output for those channels above threshold, while the chip's analog output is sent to the SVX II for digitization. The readout electronics after the SVX II is very similar to that of the silicon detector.

A cosmic ray test of a large-scale CFT prototype was carried out from 1994 to 1995 [19]. The doublet position resolution shown in Figure 2.5 is found to be less than $100 \mu \mathrm{m}$, which was consistent with expectations.

\section{Transverse Momentum Resolution}

The transverse momentum of a track is measured by its curvature in the magnetic field. The relationship between the curvature and $p_{T}$ is given by:

$$
\frac{1}{R}=0.3 \times B \times \frac{q}{p_{T}}
$$

where $R$ is the radius of curvature in meters and $q$ is the charge of the track. 


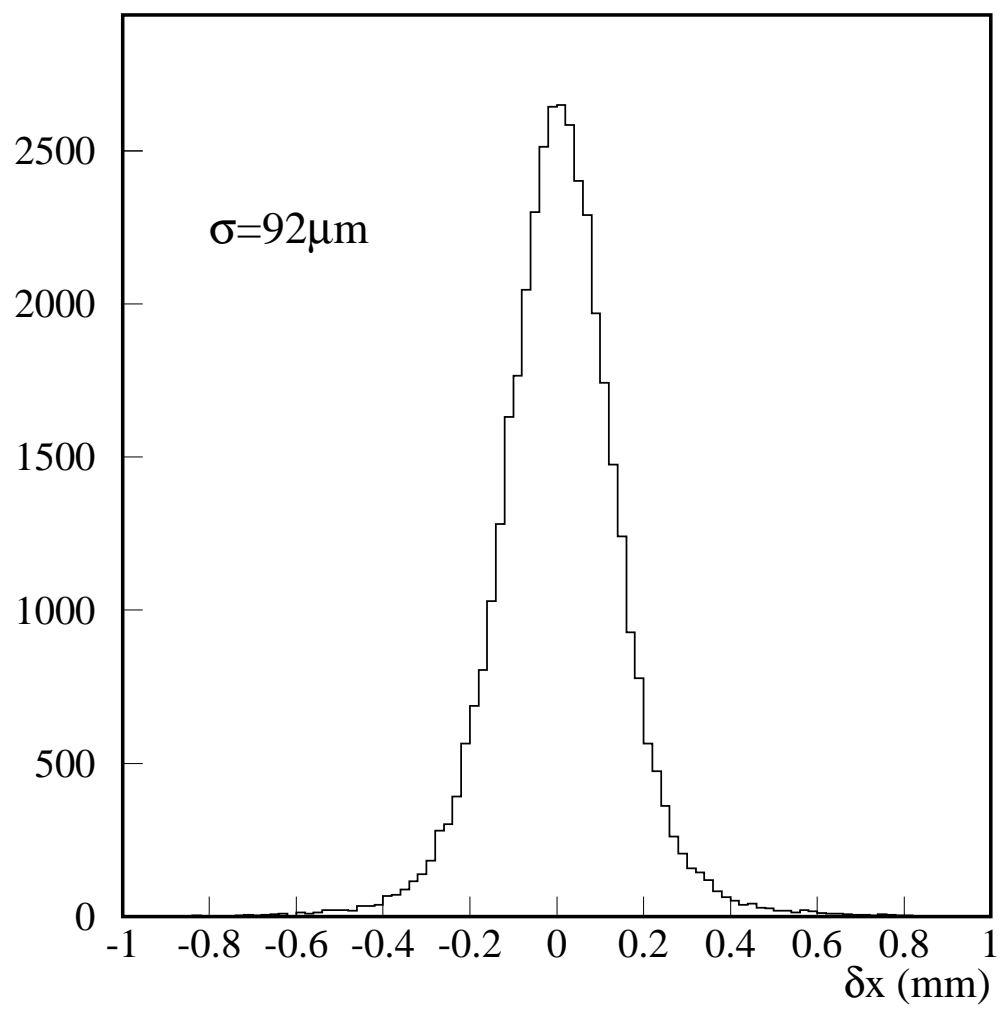

Figure 2.5: Position resolution for CFT fiber doublet from cosmic ray test. 
The uncertainty on the measured curvature has two major components: one from the multiple scattering of particles as they pass through the material of the detector layers. The other contribution is from the measurement resolution, which is determined by the individual hit resolution and the square of the measured track length. Thus, tracks measured in the SMT and CFT have significant better momentum resolution than tracks measured in either the SMT or the CFT alone.

The expected momentum resolution for the $D \varnothing$ tracking system is shown in Figure 2.6. The resolution is shown as a function of pseudorapidity for tracks of three different transverse momenta originating at $z=0$. The transverse momentum resolution at $\eta=0$ may be parameterized as [18]:

$$
\frac{\Delta p_{T}}{p_{T}}=\sqrt{0.015^{2}+\left(0.0014 \times p_{T}\right)^{2}}
$$

with $p_{T}$ in $\mathrm{GeV} / \mathrm{c}$. The worsening resolution above $|\eta|=1.6$ is caused by the loss of the full CFT coverage which starts there. The further increase in resolution above $|\eta|=2.1$ is due to the loss in coverage from the forward SMT disks.

\subsubsection{Preshower Detectors}

The Preshower Detectors are designed to aid electron identification and triggering and correct the measured electromagnetic energy for effects of energy loss in the solenoid. The detector functions as a calorimeter by early energy sampling and as a tracker by providing precise position measurements. At DØ, 


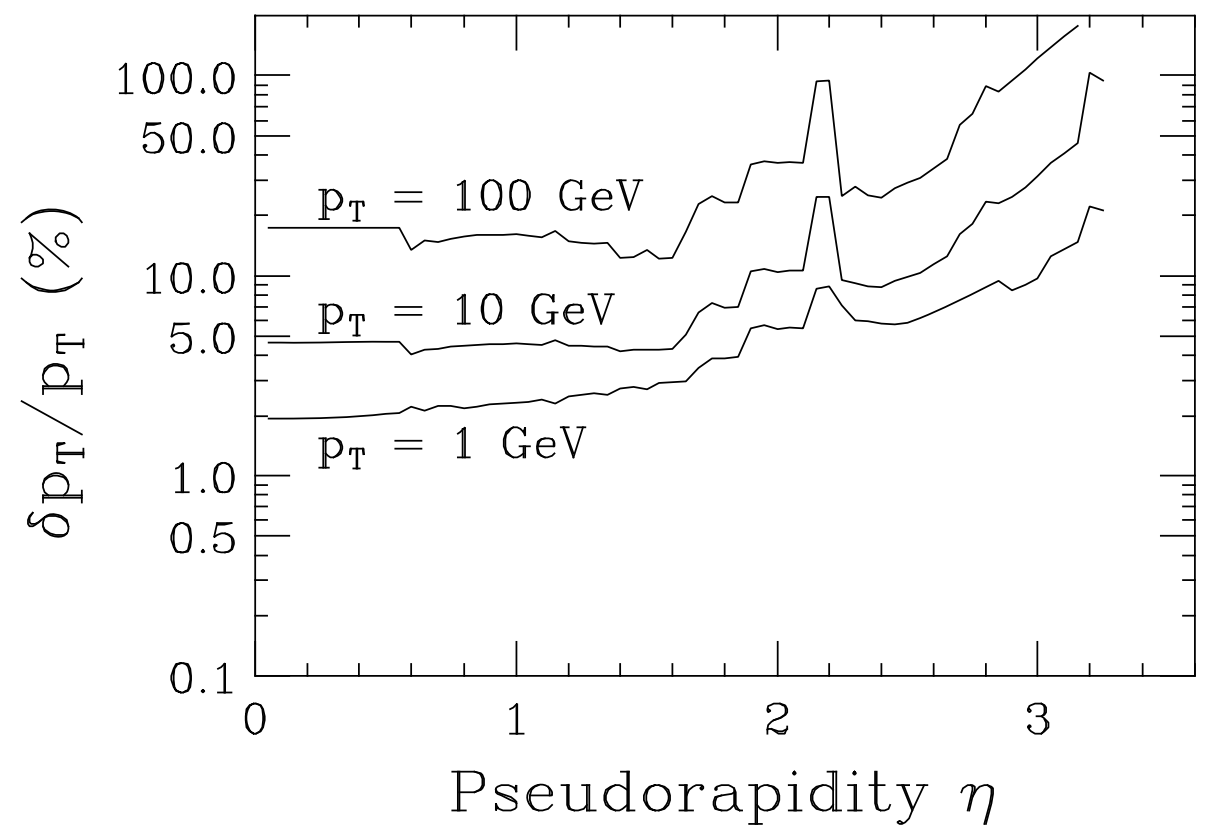

Figure 2.6: The expected $p_{T}$ resolution as a function of $|\eta|$ for the D $\varnothing$ central tracking system. 
two preshower detectors cover the central and the forward detector regions respectively.

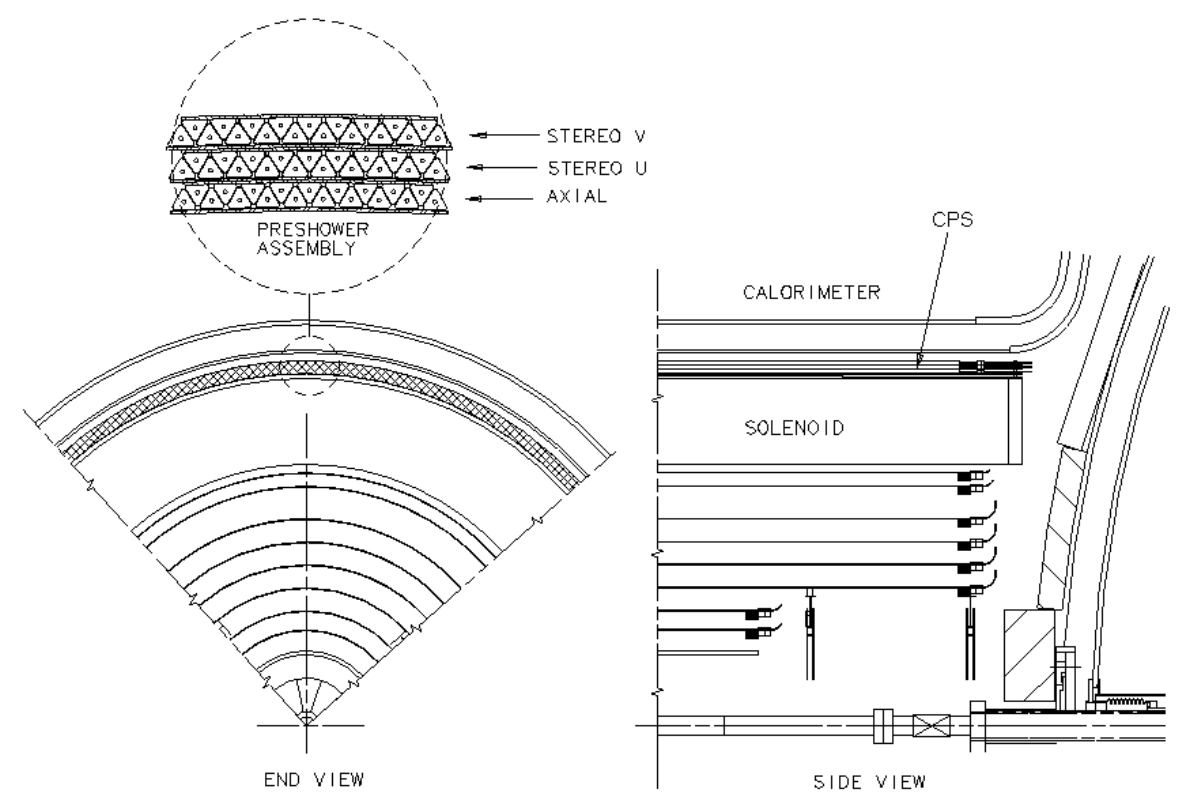

Figure 2.7: End view and side view of the CPS detector.

\section{Central Preshower Detector}

The cylindrical Central Preshower Detector (CPS) [21] is located in the $51 \mathrm{~mm}$ gap between the solenoid coil and the central calorimeter cryostat at a radius of $72 \mathrm{~cm}$, covering the region $|\eta|<1.2$. Figure 2.7 shows the cross sectional view and side view of the detector. The CPS detector consists of a tapered lead absorber and three layers of scintillator strips with wave-lengthshifting (WLS) fiber readout. The lead absorber is tapered along the beam direction to make the solenoid plus additional lead approximately equal to 2 
radiation lengths for particle trajectories at all $\eta$. The innermost layer of the CPS is an axial layer and outer two layers are $\mathrm{u}$ and $\mathrm{v}$ stereo layers with \pm $23^{\circ}$ stereo angle. The scintillator strips have a triangular cross-section with a base of about $7 \mathrm{~mm}$ and has a $1 \mathrm{~mm}$ diameter hole in the center for housing the WLS fiber. WLS fibers are connected to clear waveguides to transport the scintillation light to VLPC's and are split/mirrored at $z=0$ to halve the occupancy. Since the axial layer of CPS is treated as the 9th layer of the CFT for purpose of level 1 triggering, each CPS layer is physically divided into 8 octants with 10 sectors each ( 80 sectors in total) in $\phi$ to match the CFT trigger sectors. Due to the overall similarity, the CPS uses the same VLPC readout system as the CFT.

\section{Forward Preshower Detector}

The Forward Preshower Detector (FPS) [22], resembles its counterpart in the central region and covers the forward detector region. The FPS uses same technology as that of CPS: triangular scintillation strips with embedded wavelength-shifting fibers and readout by VLPC's.

Two FPS detectors, covering the north and south end of the detector, are mounted on the inner face of the two End Calorimeter (EC-North and ECSouth). Each detector, shown in Figure 2.8, is composed of a 2 radiation length lead layer sandwiched between two scintillator planes (a "MIP" layer sensitive to minimum ionizing particles upstream of the lead conversion layer and a shower layer downstream of the lead conversion layer). Due to the presence of the solenoid in $1.4<|\eta|<1.6$, the lead is tapered and a MIP layer coverage is 


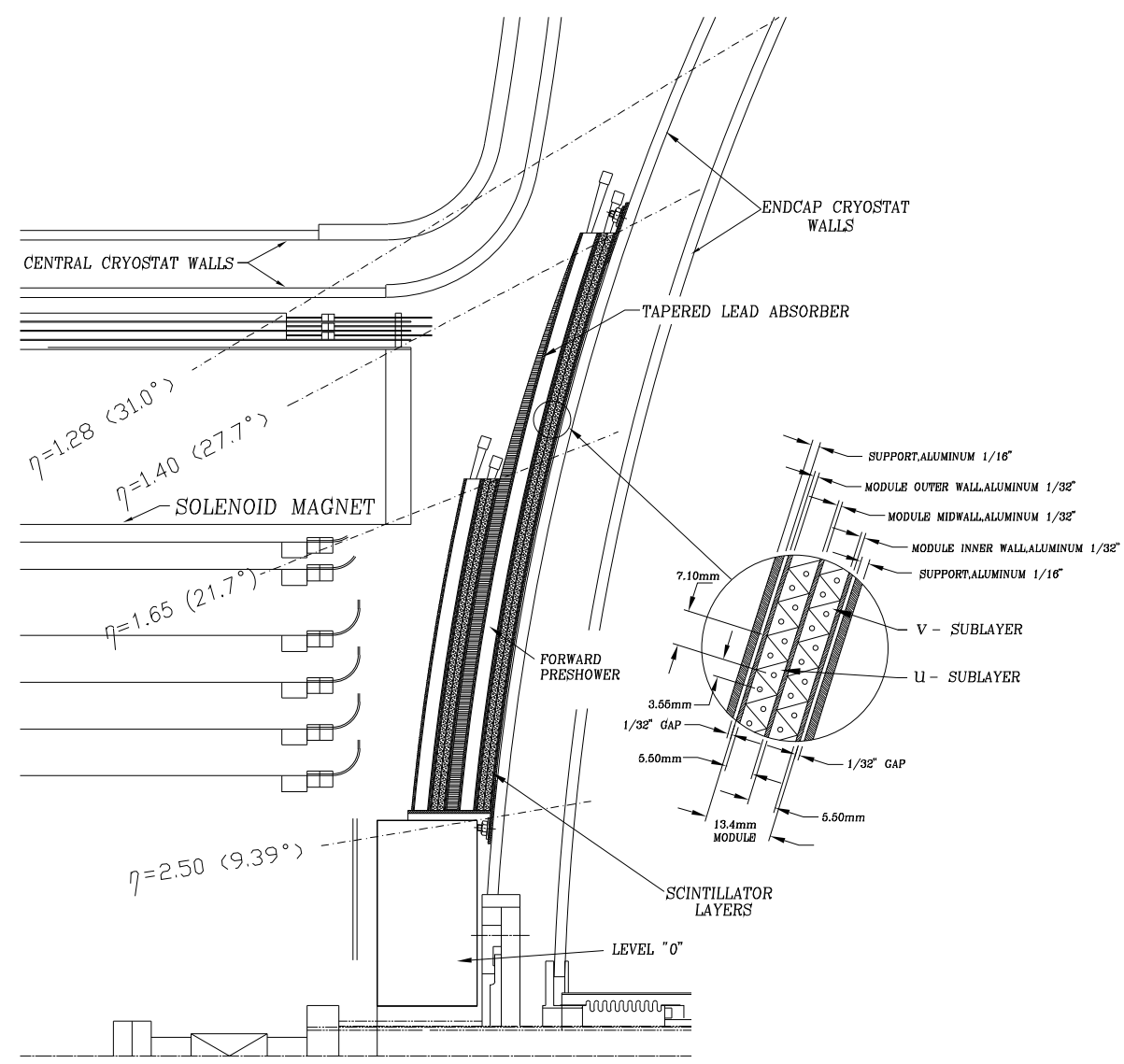

Figure 2.8: Side view of the FPS detector. 
not useful in this $\eta$ region Therefore, The MIP(shower) scintillator layer covers the pseudorapidity range $1.6<|\eta|<2.5(1.4<|\eta|<2.5)$. The FPS uses the same VLPC readout system as the CFT as well.

\subsubsection{The Calorimeter System}

At DØ, Uranium-Liquid Argon (U-LAr) calorimeters are used to identify and measure electrons, photons, jets and muons. They play a dominant role in the measurement of the energy of all particles and, via conservation of transverse momentum, of the transverse missing energy.

\section{Calorimetry Principle}

When a high energy electron $(\gg 10 \mathrm{MeV})$ traverses through matter of high atomic number it looses its energy primarily through Bremsstrahlung. Similarly, for a high energy photon the energy loss is predominantly via the electron-positron pair production in the vicinity of the nucleus. The electrons and positrons created through pair production will in turn loose energy through Bremsstrahlung. This process continues until the energy of the secondary particles falls below a critical energy level where other energy loss mechanisms become important. The whole process forms an electromagnetic shower. The energy loss of a particle through electromagnetic interactions can be characterized by:

$$
\frac{d E}{E}=-\frac{d x}{X_{0}}
$$


where $X_{0}$ is the radiation length and is dependent on the absorbing medium. For uranium $X_{0} \approx 3.2 \mathrm{~mm}$.

When hadrons pass through matter, they loose energy via the strong nuclear interactions primarily through inelastic nuclear collisions with the atomic nuclei. These collisions produce secondary hadrons, which interact inelastically to produce a further hadron generation, and so on. This type of shower is known as hadronic shower, which develops more slowly and over longer distance than electromagnetic showers. For hadronic showers, the scale for longitudinal development is set by the nuclear interaction (absorption) length $\lambda_{A}$. For uranium, $\lambda_{A} \approx 10.5 \mathrm{~cm}$.

\section{Calorimeter Design}

There are two basic types of calorimeters used in energy measurement. One such type is called a total absorption calorimeter where the particle looses all of its energy within the absorbing material of the calorimeter. The other type of calorimeter is the sampling calorimeter. In a sampling calorimeter, a particle loses only a portion of its energy, so that effectively a sampling is made of that portion of the energy lost by the particle while traversing the absorbing material of the calorimeter. A correction, called the sampling fraction, is then used to convert the fraction of the energy measured to the full energy of the particle. Sampling calorimeters are generally composed of a dense absorbing medium, and a less dense active medium which samples the ionization produced in electromagnetic or hadronic showers.

The DØ calorimeter system [15], shown in Figure 2.9, consists of three in- 


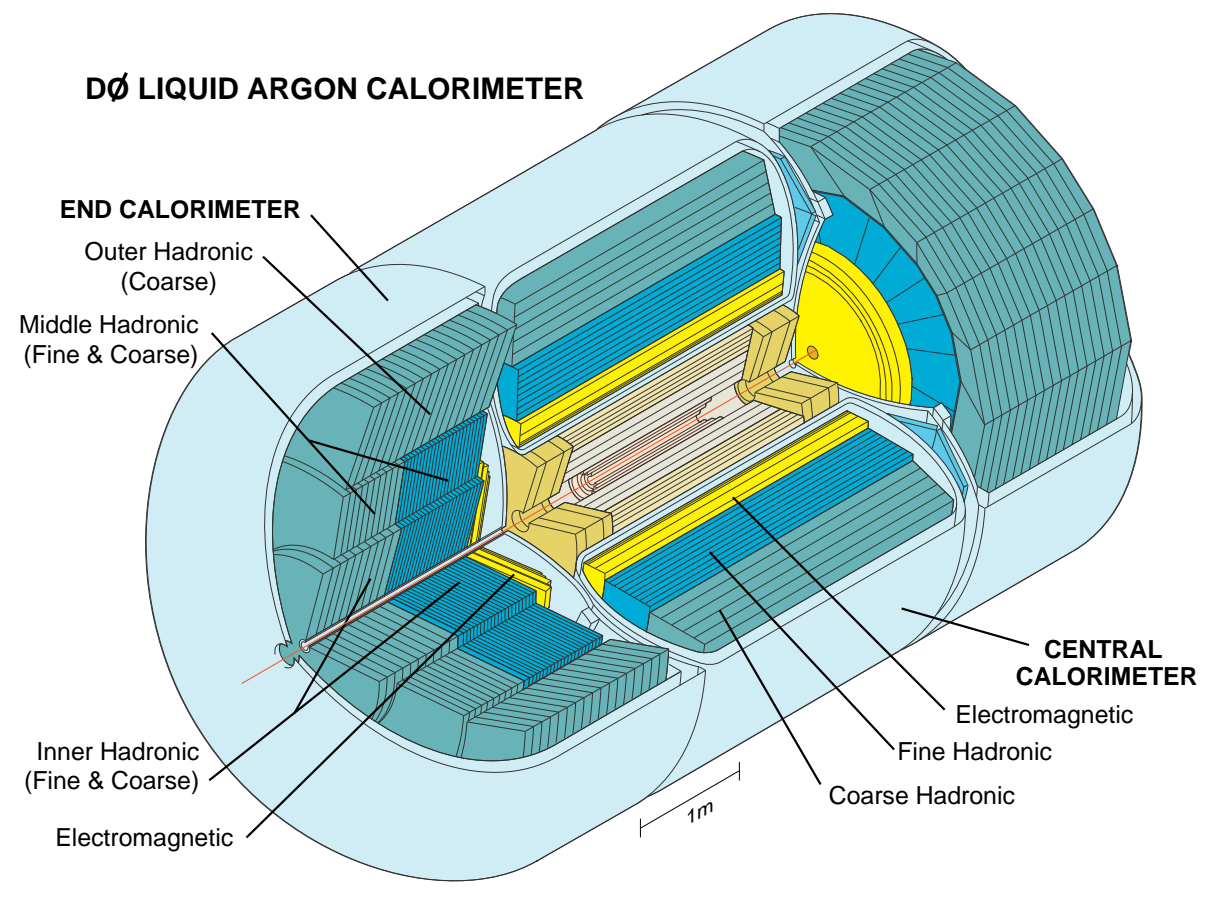

Figure 2.9: Cutaway view of the $D \varnothing$ calorimeter. 
dependent uranium-liquid argon calorimeters: one Central Calorimeter (CC) covers the region $|\eta|<1.2$, and two Endcap calorimeters (EC) extend the coverage to $|\eta| \approx 4$. These calorimeters are highly modular and finely segmented in the transverse and longitudinal shower direction. In $\mathrm{CC}$ and $\mathrm{EC}$, there are three distinct types of modules: an electromagnetic section (EM) with relatively thin and closely spaced uranium absorber plates, a fine hadronic section (FH) with thicker uranium-niobium (2\%) alloy plates and a coarse hadronic section $(\mathrm{CH})$ with either thick copper or stainless steel plates.

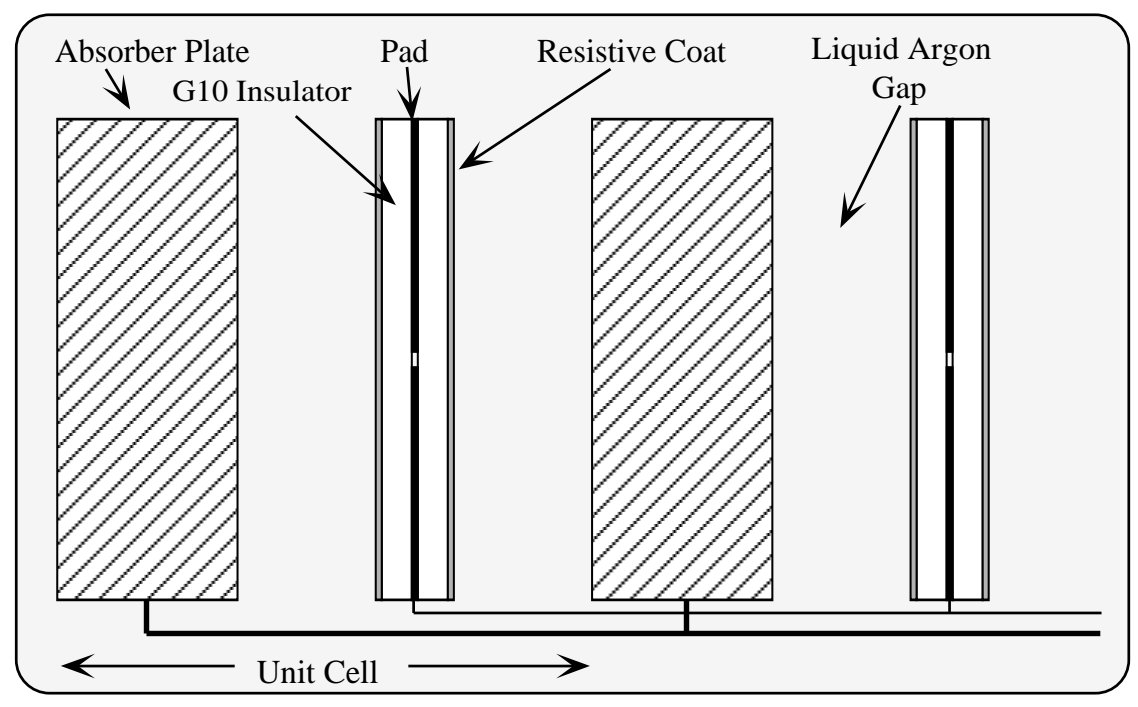

Figure 2.10: The schematic view of a calorimeter cell consisting of the absorber plates and the signal board.

Each module consists of a row of alternating absorber plates and signal readout boards as shown in Figure 2.10. The $2.3 \mathrm{~mm}$ gap separating adjacent absorber plates and signal boards is filled with liquid argon. The signal boards 
consist of a copper-clad plate pad covered by $0.5 \mathrm{~mm}$ thick G10 sheet and high resistivity carbon-loaded epoxy. An electric field is established by grounding the absorber plate while applying a positive high voltage $(\sim 2 \mathrm{kV})$ to the resistive surfaces of the signal boards. When a particle traverses the absorber plates a shower of secondary particles develops which ionizes the liquid argon. Ions liberated in the LAr drift across the gap between the absorber and signal board in about 450 ns. The resulting current induces a charge on the inner copper pads of the signal boards.

The copper clad board is milled into the pad pattern desired for segmented readout. Several such pads at approximately the same $\eta$ and $\phi$ are ganged together in depth to form a readout cell. The segmentation of the readout cells were determined from consideration of shower sizes. The transverse dimensions of the readout cell were chosen to be similar to the transverse sizes of showers: $\sim 1-2 \mathrm{~cm}$ for EM showers and $\sim 10 \mathrm{~cm}$ for hadronic showers. Furthermore, the longitudinal segmentation within the EM, FH and $\mathrm{CH}$ layers is crucial in distinguishing and separating electrons from hadrons. The segmentation was chosen to be "pseudo-projective": the centers of the readout cells lie on lines which project back to the center of the detector, but the cell boundaries are aligned perpendicular to the absorb plates. Figure 2.11 shows a quadrant of the $\mathrm{D} \varnothing$ calorimeter segmentation pattern.

\section{The Central Calorimeter}

The CC comprises three concentric cylindrical shells. There are 32 EM modules (CCEM) in the inner ring, $16 \mathrm{FH}$ modules (CCFM) in the surrounding 


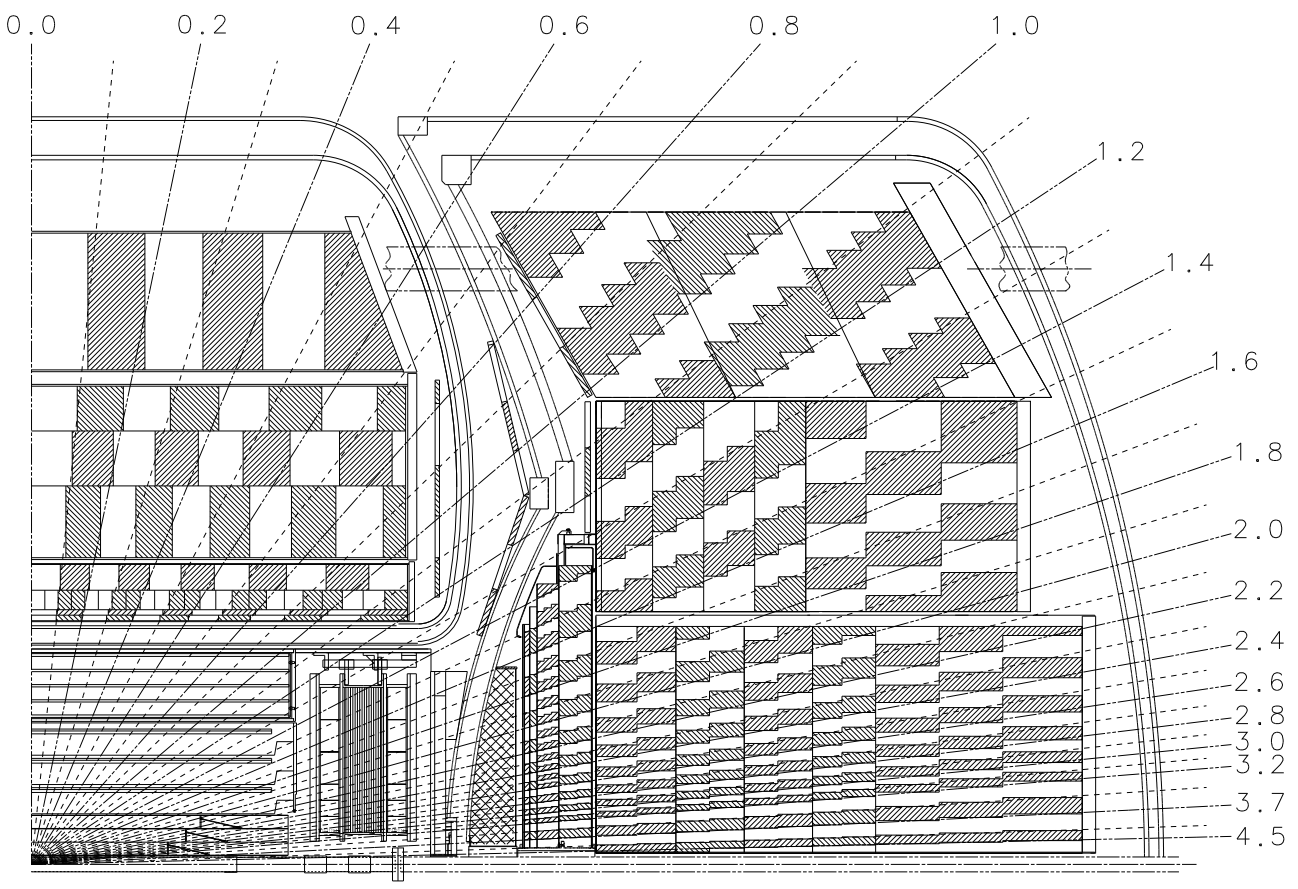

Figure 2.11: Side view of one quarter of the $\mathrm{D} \varnothing$ calorimeter showing the "pseudo-projective" segmentation pattern. 
ring, and $16 \mathrm{CH}$ modules $(\mathrm{CCCH})$ in the outer ring. The CCEM modules have 4 longitudinal gangings of signals approximately $2.0,2.0,6.8$, and $9.8 X_{0}$ in depth, with transverse segmentation as described above. The CCFH modules have 3 longitudinal gangings of signals of approximately 1.3, 1.0 and $0.9 \lambda_{A}$. The $\mathrm{CCCH}$ modules contain just one depth segment of $3.2 \lambda_{A}$. The typical transverse sizes of calorimeter cells in both EM and hadronic modules are $\Delta \eta \times \Delta \phi=0.1 \times 0.1$. The third layer of the EM modules is twice as finely segmented: $\Delta \eta \times \Delta \phi=0.05 \times 0.05$, in order to allow more precise position measurement in the region of maximum EM shower energy deposition.

\section{The Endcap Calorimeter}

The EC's are located on either side of the CC, covering the region 1.0 $<|\eta|<4.0$. Each of the two end calorimeters (ECN and ECS) contains 1 EM module (ECEM), 1 inner hadronic module(ECIH), 16 middle hadronic modules (ECMH) and 16 outer hadronic modules (ECOH)

The ECEM module has a total thickness of $24.1 \mathrm{~cm}$ and a diameter of 2 $\mathrm{m}$. It is situated with it front face $1.7 \mathrm{~m}$ away from the nominal interaction point. It contains 4 readout sections at approximately $0.3,2.6,7.9$ and 9.3 $X_{0}$. The ECIH module is of cylindrical shape. The inner radius is $3.92 \mathrm{~cm}$ while the outer radius is $86.4 \mathrm{~cm}$. It has a fine hadronic and a coarse hadronic portion. The fine and coarse hadronic portion contains four (1.1 $\lambda_{A}$ each) and one $\left(4.1 \lambda_{A}\right)$ readout sections respectively. Surrounding the ECIH is the ring of 16 modules that comprise the ECMH. Each of the ECMH modules has 4 fine-hadronic sections $\left(0.9 \lambda_{A}\right.$ each) and 1 coarse hadronic section $\left(4.4 \lambda_{A}\right)$. 
The outer ECOH modules consists of 3 coarse hadronic layers and incline at an angle of about $60^{\circ}$ with respect to the beam axis.

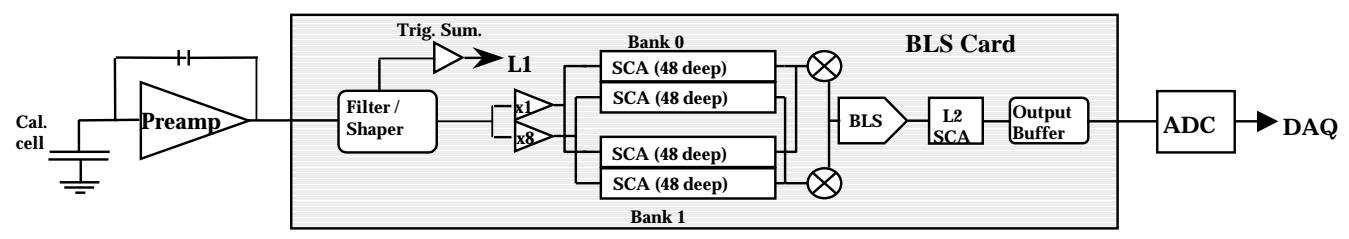

Figure 2.12: Schematic of the calorimeter readout electronics

\section{Calorimeter Readout}

Figure 2.12 shows the schematic of the calorimeter readout electronics. The signal from each readout cell is brought to a feed-through port on a $30 \Omega$ coaxial cable. From the feed-through ports the signals are carried to the preamplifier inputs on $115 \Omega$ twist-and-flat cables. The preamplifier output pulse, which is proportional to the charge received from the calorimeter cell, is delivered to the shaper and baseline subtractor (BLS) system. The preamplifier output is differentiated with a time constant of $250 \mathrm{~ns}$ by the shaper in order to be able to extract time information from the signal, enough to assign it to a given bunch crossing. The shaper output is sampled at its peak value occuring at about 320 ns and the sampled voltage is buffered in an analog pipeline which consists of a Switched Capacitor Array (SCA). Following the level 1 trigger decision, the analog signal is passed on for further processing. To remove slowly varying offsets and pileup of minimum bias events from neighboring crossings, 3 samples (396ns) before the trigger are taken as baseline. The 
baseline subtracted output is provided by the BLS circuit and stored in a parallel SCA pipeline. Following the level-2 accept, the output is sent to ADCs for digitization and the DAQ system.

\section{Calorimeter Performance}

Calorimeter modules were tested and initially calibrated by a test beam before the final assembly stage. Its response to single electrons and pions, with energies from $10 \mathrm{GeV}$ to $150 \mathrm{GeV}$, is found to be linear within $0.5 \%$. The energy resolution is measured to be [15]:

$$
\begin{array}{ll}
\text { For electrons: } & \left(\frac{\sigma_{E}}{E}\right)^{2}=(0.3 \%)^{2}+\frac{(15 \%)^{2}}{E} \\
\text { For pions : } & \left(\frac{\sigma_{E}}{E}\right)^{2}=(4.0 \%)^{2}+\frac{(45 \%)^{2}}{E}
\end{array}
$$

In Run II, because of the additional material (coils, CPS) present in front of the calorimeter, the resolutions are expected to be worse. The electron energy resolution is measured by fitting

$$
\frac{\sigma_{E}}{E}=\sqrt{C^{2}+\frac{S^{2}}{E}}
$$

to the observed $Z \rightarrow e e$ width $\left(m_{Z}=91.2 \mathrm{GeV} / \mathrm{c}^{2}\right)$ and $\Upsilon \rightarrow e e$ width $\left(m_{\Upsilon}\right.$ $\left.=9.5 \mathrm{GeV} / \mathrm{c}^{2}\right)$ in the data. Preliminary results are $C \sim 5.5 \%$ and $S \sim 29 \%$ $\mathrm{GeV}^{1 / 2}[24]$ However, the resolution is expected to be greatly improved by using information from the preshower detectors and by optimizing the calorimeter response, topics that are actively being worked on. 


\section{The Inter-Cryostat Detector}

The gap between the central and end calorimeters, spanning the region $1.1<|\eta|<1.4$, would compromise the hermeticity of the calorimeter if left uninstrumented. In the gap, two different types of detector have been installed to supplement the D $\varnothing$ calorimeter system. One system is the Inter Cryostat Detector(ICD) [25]. Two ICDs are mounted on the inner face of the north and south End Cryostats respectively. Each of the ICD detectors consists of a single layer of scintillating tiles with segmentation of $0.1 \times 0.1$ in $\eta$ and $\phi$. Wavelength shifting fiber are embedded in the tiles and long clear fibers are used to pipe the scintillation light to phototubes. The other supplementary system is a collection of modules referred to as the Massless Gap (MG). The MG modules consists of single cell structures and are mounted in the LAr on the large $\eta$ surfaces of the CCFH, ECMH, and ECOH modules. The size of these cells match those of the ICD and calorimeter.

\subsubsection{Muon Detector}

The DØ muon spectrometer [26] shown in Figure 2.2 consists of solidiron toroidal magnets, proportional drift chambers (PDT's), mini-drift tubes (MDT's) and trigger scintillation counters. The magnetic field is about 2 Tesla along the $\phi$ direction so the bend of muon tracks is approximately in the $r-z$ plane. The Wide Angle Muon System (WAMUS) covers the central region $(|\eta|<1.0)$ and the Forward Angle Muon System (FAMUS) covers the forward region $(1.0<|\eta|<2.0)$. Each system is divided into three separate 
layers, designated as A layer, B layer and C layer. A layer is inside the toroid magnet and $\mathrm{B}$ and $\mathrm{C}$ layers are outside the magnet. By measuring the bend of a muon track from hits in these 3 layers, we obtain a measurement of a muon's momentum which is used to match with the tracks measured in the inner tracking system. 


\section{Chapter 3}

\section{Data Acquisition and Event Reconstruction}

Proton anti-proton collisions occur at a very high rate at $D \varnothing$ intersection, but the majority of the events are low $p_{T}$ and not interesting for this and most other analysis. In this chapter a brief description is given on how our interesting events are selected (Trigger) and written to the storage medium (Data Acquisition System). Once the events are recorded, they are processed by a sophisticated event reconstruction program package that converts detector signals into physical objects, i.e. particle four-vectors. We describe the D $\varnothing$ event reconstruction program in the second part of this chapter.

\subsection{DØ Trigger and Data Acquisition}

The D $\varnothing$ trigger and data acquisition (DAQ) system [27] consists of four successively more selective trigger levels:

- Level 0 (L0): a hardware trigger used to detect inelastic p $\bar{p}$ collisions.

- Level 1 (L1): a hardware trigger using tracking, calorimetry and muon 
detectors. It is capable of handling a maximum $7 \mathrm{MHz}$ input rate and has an accept rate of $5-10 \mathrm{kHz}$.

- Level 2 (L2): a hardware trigger refining and combining level 1 output with a pre-processor and a global processor. It has a maximum input rate of $10 \mathrm{kHz}$ and a maximum accept rate of $1 \mathrm{kHz}$.

- Level 3 (L3): a software filter stage with partial event reconstruction on a computer farm. It has a maximum accept rate of $50 \mathrm{~Hz}$.

In the following, we briefly discuss these components of the trigger system.

\subsubsection{Level 0 and the Luminosity Monitor}

The primary purpose of level 0 is to detect non-diffractive inelastic collisions with high efficiency and to make accurate luminosity measurements. L0 consists of two pixel arrays of plastic scintillator counters located on the inside faces of the end cryostats and arranged symmetrically about the beam pipe, covering the region $2.7<|\eta|<4$.4. The scintillation light is read out using fine-mesh photo-multiplier tubes attached directly to the pixels.

In addition, the difference in arrival time for particles hitting the L0 detectors on either side of the interaction point is used to provide fast determination of the $z$-coordinate of the event vertex for use in next levels of triggers. L0 also provides diagnostic information regarding the performance of the accelerator. 


\subsubsection{Level 1}

The L1 makes trigger decisions in less than $4.2 \mu$ s with zero dead time. Figure 3.1 shows the structure of the level 1 and level 2 trigger. The L1 trigger detectors include the CFT, CPS, FPS, the calorimeter, and the muon scintillators and drift chambers. The calorimeter, fiber tracker, and preshower detectors provide electron triggering for $|\eta|<2.5$ while the fiber tracker and muon systems provide muon triggering in the region $|\eta|<2.0$. The L1 triggers associated with each of the trigger detectors examine each event and report their findings or trigger terms to the L1 Framework (L1FW). The L1FW supports 128 unique L1 trigger bits and each bit requires a unique combination of trigger terms. A series of Field Programmable Gate Arrays (FPGA's) examines the list of terms collected from the L1 trigger detectors to determine if a specific L1 bit has been satisfied. If so, the L1FW issues a "L1 accept" and the event data is digitized and moved into a series of 16-event deep pipeline buffers to await a L2 trigger decision.

\subsubsection{Level 2}

The L2 trigger, comprised of hardware engines associated with specific detectors and a single global processor, tests for correlation between L1 triggers. The L2 makes trigger decision within $100 \mu$ s with deadtime less than $5 \%$.

There are two L2 stages to test for correlations among L1 triggers. In the first stage, or preprocessor stage, the L1 trigger information is retained and transformed into physical objects such as energy clusters or tracks. In 


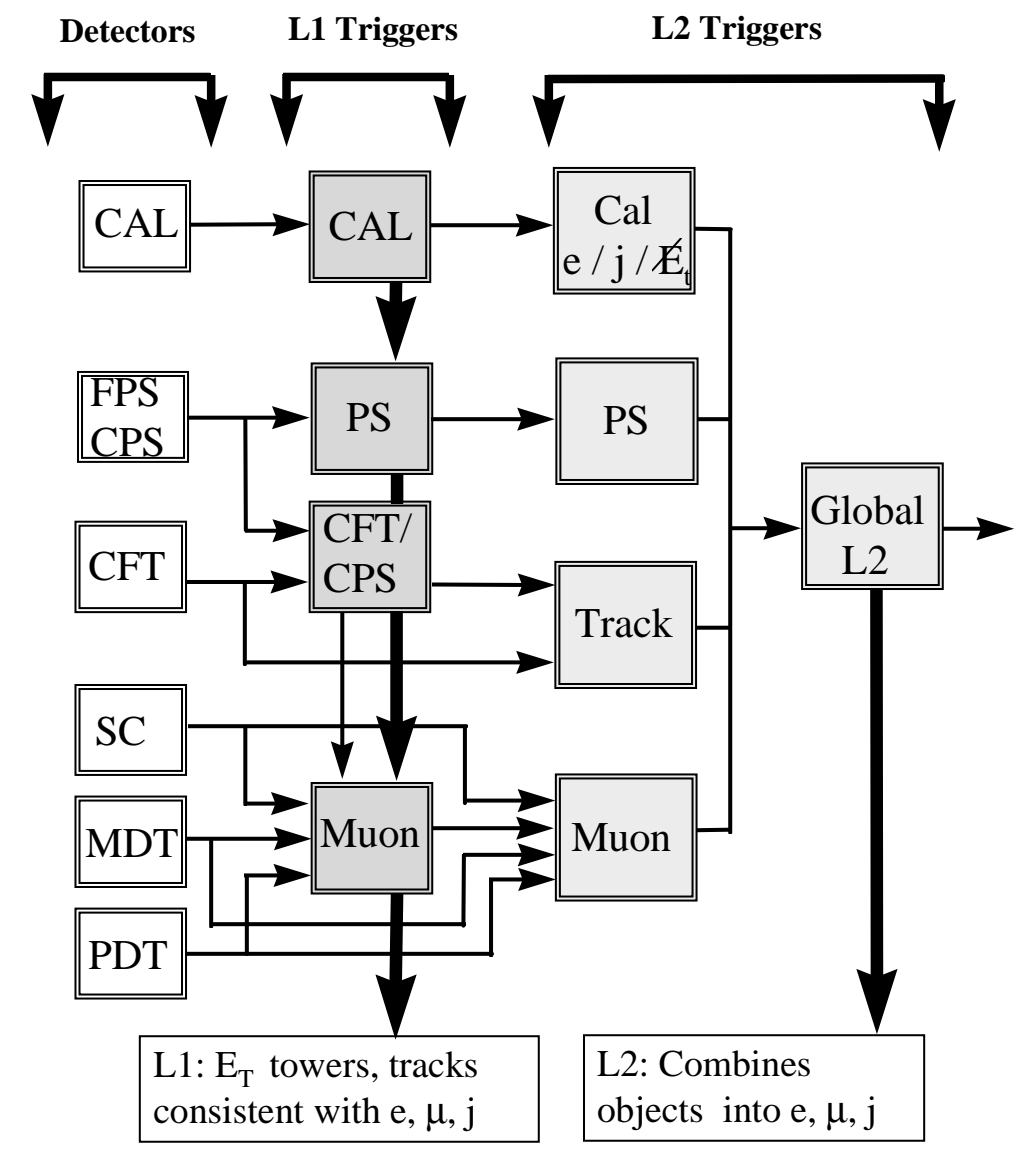

Figure 3.1: The structure of the level 1 and level 2 triggers. The horizontal arrows denote information flow.

the second global stage, a global processor makes trigger decisions based on correlations among the physical objects from different detectors. A L2 framework (L2FW), utilizing the same FPGA's as the L1 Framework reports trigger decisions to L3. 


\subsubsection{Level 3 and Data Acquisition}

The DØ data acquisition system and the level 3 trigger hardware are closely intertwined and are discussed together in this section. Figure 3.2 shows the RunII data acquisition path. Upon receipt of a L2 accept from the global processor, L3 will initialize detector readout and move the event data into 8 L3 transfer buffers. From the buffers the data is loaded onto one of 16 high speed data cables. The data cables carry the data to a VME Receiver Collector (VRC) which sends the data onto the L3 Farm Segment Controllers (SC's). Each SC examines passing data blocks and moves the data blocks belonging to a single event into one of the 64 L3 processor nodes for L3 triggering. Software filters in the L3 nodes consist of the combinations of the physics tools written in $\mathrm{C}++$. These tools have access to all event data to search for electron, muon, jet candidates and other physics objects. Any event meeting filter requirements will be transferred to tape storage for offline reconstruction. The Level 3 has a maximum event accept rate of $50 \mathrm{~Hz}$.

\subsection{Offline Event Reconstruction}

The raw event data from the detector is in the form of digital signal such as charges, pulse heights, etc. However, in physics analysis one studies objects like jets, electrons and photons which are characterized by particle ID, four momenta, chisquare etc.. The process of converting raw data into the final physics objects is called "Event Reconstruction". At DØ, event reconstruction is performed by the DØreco program [28] which performs the following tasks: 


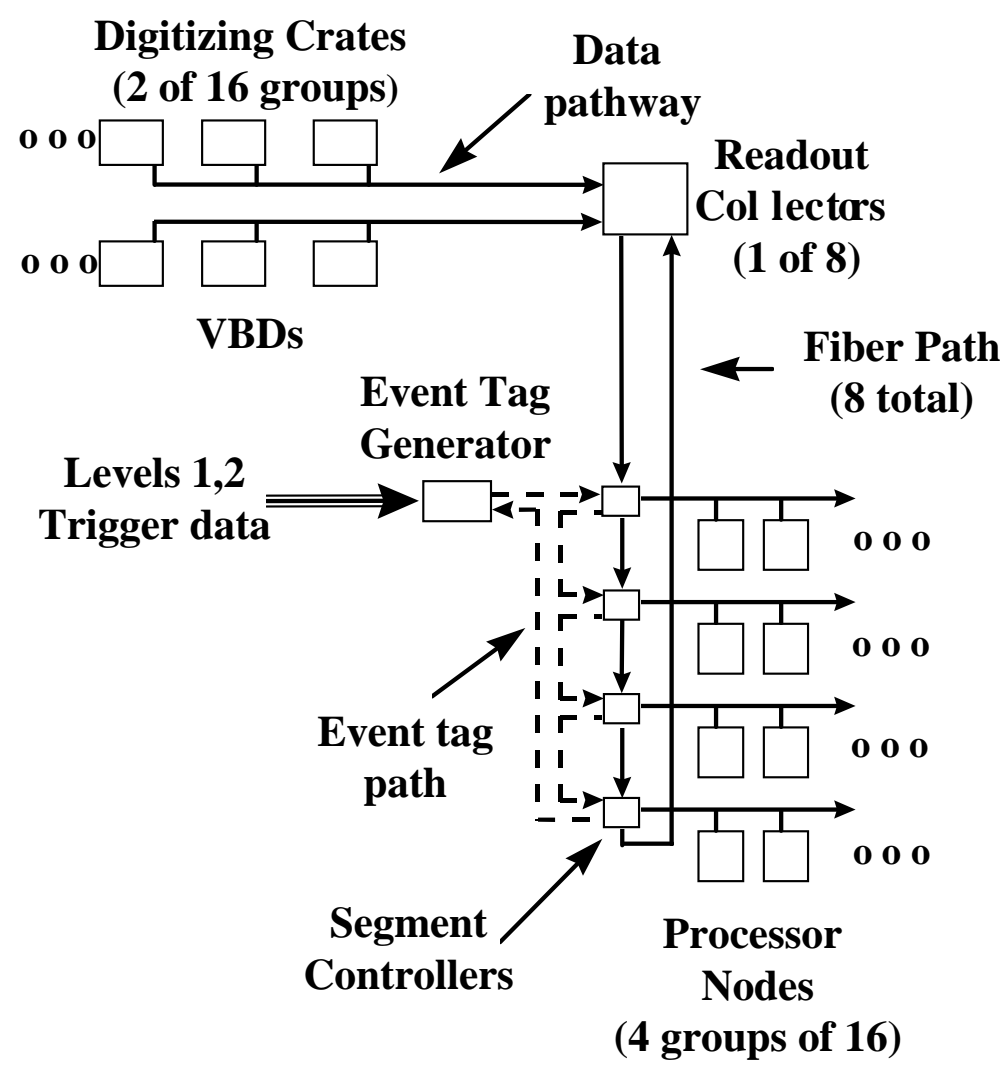

Figure 3.2: Level 3 framework. Data flow is indicated by the direction of the arrow.

- Hit finding, digitized signals from tracking layers are converted into spatial locations of hits. Signals from calorimeter cells are converted to energy depositions

- Tracking and clustering, where the tracking detector hits are joined together to form tracks and the calorimeter energy depositions in the cells are grouped to form clusters. 
- Particle identification, the tracking and calorimeter information are combined to form candidates for electrons, photons, jets and muons.

The criteria applied by DØreco in choosing candidates are quite loose. In the following sections, we discuss the details of the reconstruction of some of the physics objects that are most relevant to this analysis. These details are valid only for the p13 version of the DØreco program which was used in this analysis.

\subsection{Track Reconstruction}

Reconstruction of the signals from each SMT and CFT layers yields hits, which contain a position measurement with errors. Track reconstruction programs use these hits to find tracks and calculate their tracking parameters $\left(\eta, \phi\right.$, and $\left.q / p_{T}\right)$ and the full error matrix at the point of closest approach (DCA). Since only the central calorimeter is fully covered by the tracking system, the discussion here is mainly focused on the track reconstruction in the central detector region.

Two tracking algorithms are employed in p13 DØReco: the Global TRacking (GTR) algorithm and the Histogramming Track Finding (HTF) algorithm.

\subsubsection{The GTR Tracking Algorithm}

The GTR tracking algorithm [29] is a road-following algorithm which uses specific paths (roads) during track finding. The basic GTR components are: 
- Surfaces: GTR builds a model of the tracking detectors using abstract surfaces. The specific types of surfaces needed to describe the D $\varnothing$ detector are cylinders for the fiber tracker and $\mathrm{x}-\mathrm{y}$ and $\mathrm{z}$ planes for the silicon detector. Track's direction and curvature and their errors are calculated for each surface.

- Paths: the actual track finding is controlled by path. The paths are an ordered list of the surfaces that a particle coming from a $\mathrm{p} \overline{\mathrm{p}}$ collision would cross. The first few surfaces are used to build a "seed track" with approximate parameters and errors

- Propagators: to extrapolate the seed tracks to the remaining surfaces, propagators are used. A track propagator solves the equation of motion for a track, including the effect of magnetic fields. The propagator updates the track error for the effects of multiple scattering and energy lost in any material crossed while reaching the target surface.

- Fitters: once the track reaches a new surface, fitters attempt to add a new hit to the track. The fitters combine the track and hit errors into a match $\chi^{2}$ and reject the hit if the $\chi^{2}$ is too high. If the hit matches and is added to the track, the fitters update the track parameters and errors. It is also possible for a track not to find a hit on a particular surface. GTR will store such missed surfaces and the probability for the miss to occur with the track information.

- Filters: After moving through all the surfaces in a particular path, a number of filters are applied to clean the list of candidate tracks. 
A default central path is defined for tracks in the central detector region. To illustrate how GTR finds tracks with the default central path, we explain the 3 stages involved: axial fiber tracking, stereo fiber tracking and silicon extension.

- The first stage is to build seed tracks from combinations of hits in the outer three CFT axial layers. The seed tracks are required to come from the center of the detector and have a minimum transverse momentum of $0.4 \mathrm{GeV} / \mathrm{c}$. The seed track's direction and curvature are calculated, along with the corresponding errors.

A propagator can now use the track parameters to extrapolate the track through the remaining five axial fiber layers, with fitters adding hits at each layer. If more than one hit has a reasonable match $\chi^{2}$ value, multiple tracks are produced, one for each hit. After passing all eight axial fiber layers, the list of tracks is filtered to remove duplicate tracks.

- The surviving tracks are passed to the next stage of the processing, which looks for hits in the stereo layers of the fiber tracker. It requires two hits coming from the outer two stereo fiber layers and allows up to 2 misses out of the 8 stereo layers. Track finding proceeds as before, with propagators and fitters used to build the tracks, which are filtered after reaching the innermost fiber layer.

- The final step takes the remaining tracks and attempts to add hits in the silicon detector. Unlike in the fiber tracker, a track is allowed to miss any given silicon layer. The only requirement is that the track needs to 
pick up at least 4 hits in the silicon detector. After these 3 stages, a complete central track is established.

To increase the tracking efficiency for the GTR algorithm, the default central path was modified to include tracks made only from axial fibers, rather than requiring the stereo fibers and silicon hits. Also, tracks found in the silicon detector are also extended into the fiber tracker, only requiring to pick up at least 7 fiber hits.

\subsubsection{The HTF Tracking Algorithm}

The HTF tracking algorithm [30] starts with hit preselection using histogramming technique based on the Hough Transform which significantly reduces the possible combinations. The output of the preselection stage is a set of templates. Each template is a track candidate consisting of several hits, with approximately known track parameters. The templates are further processed with the Kalman filter which discards fake templates, removes wrong hits, and calculates track parameters accurately. The implementation of the algorithm makes use of two strategies:

- Strategy A: Track finding begins with SMT hits. It removes templates with less than 4 SMT hits. Then it builds 3D SMT tracks and tries to extrapolate SMT tracks to the CFT to complete tracks.

- Strategy B: Track finding begins with CFT axial hits. It removes templates with less than 7 axial hits and builds 2D CFT tracks. Based on the 2D CFT tracks, it makes all possible 3D CFT tracks by adding 
stereo hits. Finally, it tries to extrapolate 3D CFT tracks to the SMT and completes tracks.

\subsubsection{Final Track Candidates}

To maximize the track finding efficiency, track candidates found in the GTR and HTF tracking algorithms are merged to form the final list of track candidates for the event. During the merging, duplicate tracks sharing more than $50 \%$ of their hits with another track are identified and removed. The decision on which track to keep is done in two steps. First, the length of the two tracks are compared and the longer track is kept. Second, if two tracks are the same length, then the track with the lowest $\chi^{2}$ is kept.

In the final list of track candidates, the following types of tracks are presented:

- "Global" tracks, that include

- axial + stereo fiber tracks extended into the silicon detector, adding at least 4 more hits.

- axial + stereo fiber tracks, 16 CFT hits for the track

- tracks found in the silicon, extended into the fiber tracker and has at least 7 or more axial hits.

- "Axial only" track, that has only 8 CFT axial hits for the track

- "SMT only" tracks, that has 4 or more SMT hits for the track 
Among the 3 types of tracks, the "Global" tracks have the best measured $\eta, \phi$, and $q / p_{T}$. The "Axial only" tracks has similar quality as the "Global" tracks except that they have no $\eta$ information. The "SMT only" tracks has precise measurement of the $\eta$ and $\phi$, but has a relatively poor measurement of $q / p_{T}$

\subsection{Vertex Reconstruction}

From the reconstructed tracks, primary and secondary vertex are found. The primary vertex is the actual interaction point of the beams. The $\mathrm{x}$ and $\mathrm{y}$ positions of the primary vertex are close to zero due to the small cross sectional size $(\approx 40 \mu \mathrm{m})$ of the beam. However, the $\mathrm{z}$ position of the primary vertex is distributed with a $\sigma_{z}=25 \mathrm{~cm}$ around $z=0$. Secondary vertices are from long live mesons such as B or D mesons. A secondary vertex is built from tracks that do not originate from the primary vertex. The vertex reconstruction at DØ uses LEP's “impact parameters" algorithm [31].

\subsection{Jet Reconstruction}

Many hard collisions involve the production of colored particles, quarks and gluons, as tree level partons. Color confinement requires that these energetic partons fragment into a spray of colorless hadrons through a process called "hadronization", and the physical manifestation of this spray of hadrons is called a jet. In $\mathrm{p} \overline{\mathrm{p}}$ physics, the standard definition of a jet has used a fixed cone 
algorithm [32]. In DØ, the fixed cone algorithm is implemented as a three-step process: preclustering, cone clustering, and a final splitting/merging phase.

- Preclustering begins with a list of calorimeter towers ordered in $E_{T}$. Starting from the highest $E_{T}$ tower, for every tower with $E_{T}>1 \mathrm{GeV}$, a precluster is formed from all adjacent towers within a window of $\Delta \eta \times$ $\Delta \phi=0.3 \times 0.3$. Preclustering continues until all the towers with $E_{T}>$ $1 \mathrm{GeV}$ are assigned to a precluster.

- The second step begins with the preclusters found in the previous stage. Starting with the highest $E_{T}$ precluster, the $E_{T}$ weighted $(\eta, \phi)$ centroid of the precluster is found and identified as the jet axis. All towers within a radius of $\mathcal{R}=\sqrt{\Delta \eta^{2}+\Delta \phi^{2}}$ are assigned to the jet.

- In splitting/merging stage, if two jets have shared calorimeter towers, a parameter called $f_{S M}$ is calculated as:

$$
f_{S M}=\frac{E_{T}^{\text {shared }}}{E_{T}^{\text {min }}}
$$

where $E_{T}^{\text {shared }}$ is the sum of the transverse energies of the common towers and $E_{T}^{\min }$ is the lesser of the transverse energies of the two jet clusters. If $f_{S M} \leq 0.5$, then two jets are made and each tower is assigned to the closest jet. If $f_{S M} \geq 0.5$, then all the towers are merged into one jet.

At $\mathrm{D} \varnothing$, there are two cone sizes available, $\mathcal{R}=0.5$ and $\mathcal{R}=0.7$. The first one is often used in electron related studies since the cone size is close to that used in electron reconstruction. The larger cone size is often used in analysis requiring more accurate jet energy. 


\subsection{Electron Reconstruction and Identification}

The typical signature of electrons in the D $\varnothing$ detector consists of an electromagnetic(EM) shower in the calorimeter, a energy cluster in the preshower detector and a track in the tracking system. Photons produce EM showers similar to electrons in the calorimeter but do not have tracks in the tracking system.

\subsubsection{EM Candidates}

The electron/photon reconstruction program at D $\varnothing$ is called EMReco. EMReco reads in a list of preclusters produced by a precluster builder program [33] and makes electron candidates out of them.

Currently, there are two preclustering algorithms used at DØ: the Simple cone algorithm (Scone) and the Cell-Nearest-Neighbor (CellNN).

- The Scone Algorithm [34] is a simplified version of the fixed cone algorithm in the jet reconstruction without the merging/splitting part. First, the highest $E_{T}$ EM towers are selected as the starting points of the preclusters. Adjacent EM towers above $50 \mathrm{MeV}$ are added to a precluster if they are within a window of $0.3 \times 0.3$ in $\eta \times \phi$ (for $\mathrm{CC}$ ) or within a cone of $10 \mathrm{~cm}$ radius in EM3 (for EC). Second, all EM towers within a cone of radius $\mathcal{R}=\sqrt{\Delta \eta^{2}+\Delta \phi^{2}}=0.4$ with respect to the precluster axis are added to the precluster. Then the axis is recalcualted and the second step is repeated until the final cluster does not change. The Scone algorithm is used in this analysis. 
- The CellNN algorithm [35] is based on calorimeter cells rather than towers. In each calorimeter layer, the cell with the highest energy is used as a seed. A precluster is formed in each calorimeter layer by merging the seed cell with its neighboring cells. This procedure repeats with the other cells left outside the existing precluster. Each precluster in EM3 initiates a global cluster and, layer by layer, preclusters matching an angular requirement are added to the global cluster. The CellNN algorithm is good for reconstructing electrons in events involving many jets in the final state since it better separates the electrons from nearby jets.

In EMReco, preclusters have to pass the following cuts in order to be used to build the EM candidate:

- more than $90 \%$ of the total energy of the precluster is deposited in the EM layers of the calorimeter

- The total transverse energy of the precluster $E_{T}>1.5 \mathrm{GeV}$

- Isolation of the precluster $<0.2$. The isolation is defined as $\frac{\text { totE-coreE }}{\operatorname{coreE}}$, where totE is the EM and hadronic energy within a cone size of $R=$ $\sqrt{\left(\Delta \eta^{2}+\Delta \phi^{2}\right)}=0.4$ centered at the precluster center, the coreE is the EM energy within a cone size of 0.2 .

For each EM candidate, the centroid of the preclusters is re-calculated as a weighted mean of the coordinates of the center of the cluster cells in the third layers of the EM calorimeter

$$
\vec{x}_{c l u s}=\frac{\sum_{i} w_{i} \vec{x}_{i}}{w_{i} \vec{x}_{i}}
$$


where the weights $w_{i}$ are defined as

$$
w_{i}=\max \left(0, w_{0}+\ln \left(\frac{E_{i}}{E}\right)\right)
$$

where $E_{i}$ is the energy of the $i^{\text {th }}$ cell, $\mathrm{E}$ is the energy of the cluster, and $w_{0}$ is a parameter independent of $\eta$ and $\phi$ chosen to optimize the position resolution. At this point, EM candidate's $\eta$ and $\phi$ are calculated using the reconstructed primary vertex.

Since electromagnetic objects produce EM showers in the preshower detector, EMReco then looks for a matching preshower cluster for the EM candidate found in the previous steps. If there is a matching central preshower cluster within $\Delta \eta \times \Delta \phi=0.05 \times 0.05$ or a matching forward preshower cluster within $\Delta \eta \times \Delta \phi=0.1 \times 0.1$, EMReco corrects the energy and position of the EM candidate with the preshower cluster energy and position.

Finally, EMReco looks for tracks within a window of $\Delta \eta \times \Delta \phi=0.05 \times$ 0.05 around the EM candidate. If a matching track is found, then the EM candidate's directions are replaced by the track's $\eta$ and $\phi$ and do not depend on the reconstructed primary vertex anymore. The EM candidate's charge are set according to the track and assigned an ID of \pm 11 . If there is no track in the $\Delta \eta \times \Delta \phi$ window, then the EM candidate is assigned an ID of 10 . Since this track matching is very preliminary, we do not use the ID information in our analysis and instead use a more advanced tracking matching algorithm (Section 3.6.3). 


\subsubsection{Standard Electron Identification}

The majority of the electron candidates found by EMReco are not real electrons and substantial further identification is required. The parameters used to identify EM candidates as good electrons are: i) EM fraction, ii) Isolation fraction, and iii) H-Matrix $\chi^{2}$. EM candidates passing these requirements are called "loose electrons".

\section{EM energy fraction}

The EM energy fraction $\left(f_{E M}\right)$ is defined as the ratio between the energy deposited in the EM layers of the calorimeter and the total energy of the cluster including the energy deposited in the hadronic calorimeter. An electron or photon candidate must have $f_{E M}>0.90$. Figure 3.3 shows the distribution of the EM energy fraction for electrons in $Z \rightarrow e e$ events and multijet events.

\section{Isolation fraction}

Since electrons produced in the decay of $\tilde{\chi}_{1}^{ \pm} \tilde{\chi}_{2}^{0}$ are isolated, the calorimeter clusters corresponding to these electrons should be isolated as well. The isolation of an electron is measured by comparing the electromagnetic energy within a cone of radius $\mathcal{R}=\sqrt{\Delta \eta^{2}+\Delta \phi^{2}}=0.2$ centered on the electron $\left(E_{E M}(0.2)\right)$ to the total energy contained within a concentric cone of radius $0.4\left(E_{\text {Total }}(0.4)\right)$. The isolation fraction is then defined as:

$$
f_{i s o}=\frac{E_{\text {Total }}(0.4)-E_{E M}(0.2)}{E_{E M}(0.2)}
$$

Figure 3.4 shows the distribution of isolation fraction for electrons in $Z \rightarrow$ 


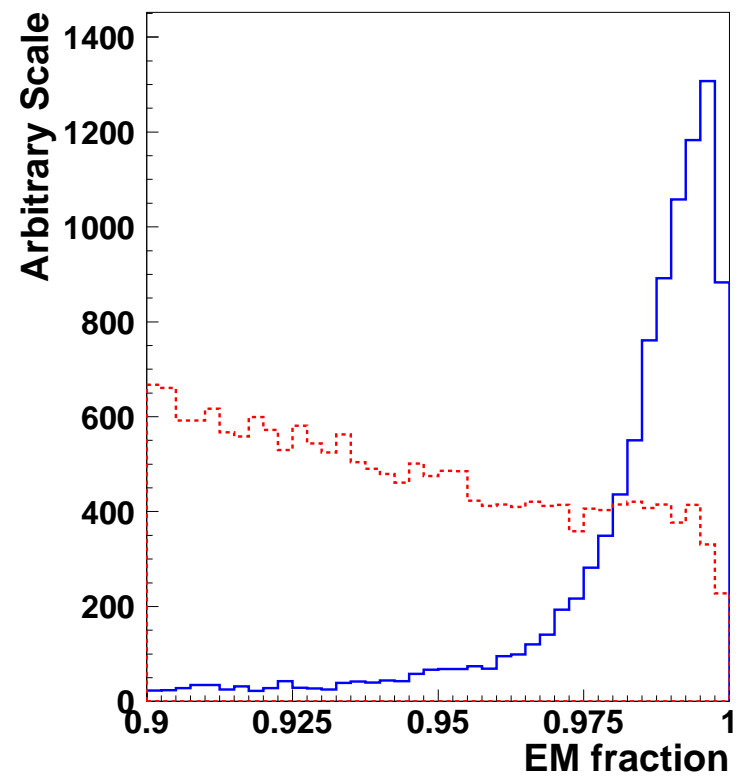

Figure 3.3: The distribution of EM fraction for electrons in $Z \rightarrow e e$ events and multijet events. The solid line is for $Z \rightarrow e e$ events and the dashed line is for multijet events.

$e e$ events and multijet events. In our analysis, EM candidates must pass the $f_{\text {iso }}<0.15$ requirement.

\section{H-Matrix $\chi^{2}$}

Electromagnetic showers are well collimated depositions of energy compared to the lumpy shape of jets. On average, the EM shower shape is the well known teardrop pattern [36]. Fluctuations cause the energy deposition to vary around the average in a correlated fashion which, for example, demands that a shower that fluctuates high in one layer will tend to exhibit negative 


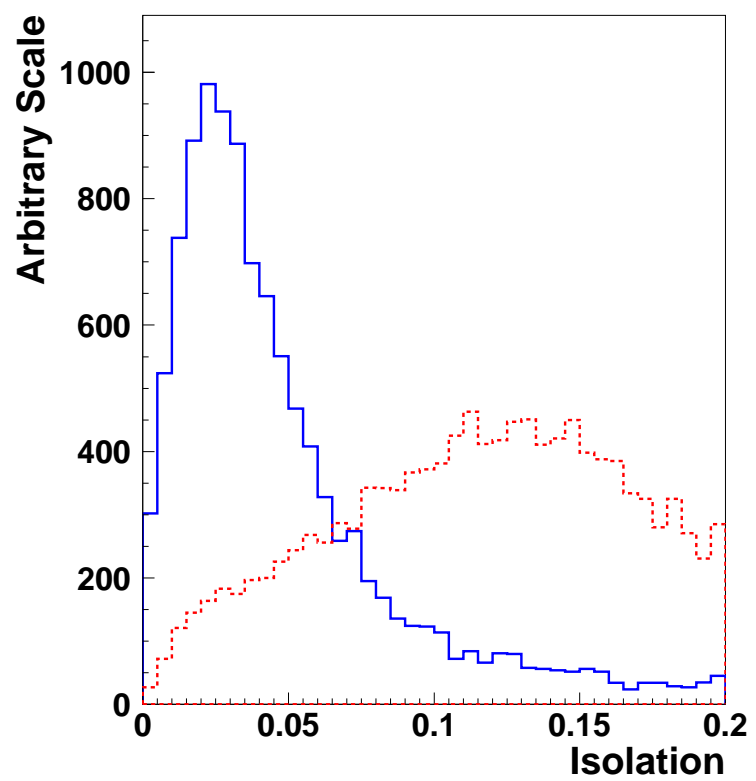

Figure 3.4: The distribution of isolation fraction for electrons in $Z \rightarrow e e$ events and multijet events. The solid line is for $Z \rightarrow e e$ events and the dashed line is for multijet events.

fluctuations in the other layers. Such patterns are well modeled by a full detector simulation such as GEANT. To determine the "electron-ness" of a shower, covariance matrices [15] are constructed using Monte Carlo electrons of various energies. For a sample of $\mathrm{N}$ simulated electrons, one can define the covariance matrix:

$$
M_{i j}=\frac{1}{N} \sum_{i=1}^{N}\left(x_{i}^{n}-\bar{x}_{i}\right)\left(x_{j}^{n}-\bar{x}_{j}\right)
$$

where $x_{i}^{n}$ is the value of observable $\mathrm{i}$ for electron $\mathrm{n}$ and $\bar{x}_{i}$ is the mean value of observable $\mathrm{i}$ for the sample. The $\chi^{2}$ which measures the consistency 
of shower $\mathrm{n}$ with an electromagnetic shower, is defined as:

$$
\chi^{2}=\sum_{i, j=1}^{N}\left(x_{i}^{n}-\bar{x}_{i}\right) H_{i j}\left(x_{j}^{n}-\bar{x}_{j}\right)
$$

where $H$ is the inverse of the covariance matrix M. Several dimensions of H-Matrices have been studied and their performance compared. In current EMreco, an 8-dimentional H-Matrix is used. The eight observables used are:

- Longitudinal shower shape variables: Fractions of shower energy in the first, second, third and fourth EM layer of the calorimeter

- Transverse shower shape variables: $S_{1}$, size of the cluster along the z-axis for a central EM cluster or the r-axis for forward EM clusters. $S_{2}$, size of the cluster along the $r-\phi$ axis

- Logarithm of the total shower energy

- Position of the event vertex along the $\mathrm{z}$ axis

Figure 3.5 shows the distribution of the $\chi_{H M x 8}^{2}$ for electrons in $Z \rightarrow e e$ events and multijet events. In this analysis, the EM candidate's $\chi_{H M x 8}^{2}$ is required to be less than 20 .

\subsubsection{Electron Track Matching}

A significant source of background to real electrons is from photons produced directly or by the decay of a $\pi^{0}$. Such photons are not expected to have a matching track in the central detector but due to the accidentally overlapping tracks from nearby charged particles they may still be identified as electrons. 


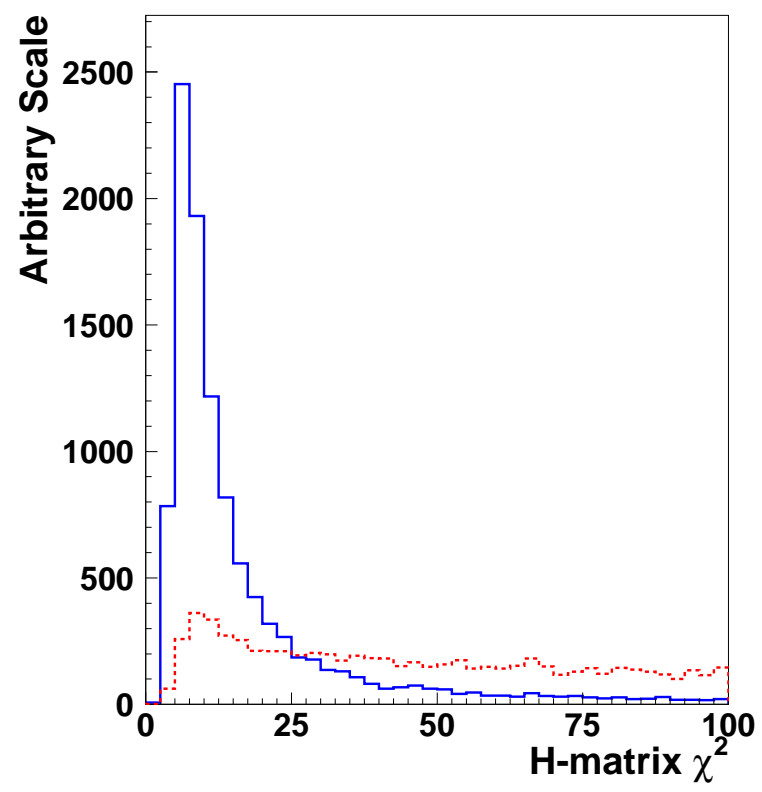

Figure 3.5: The distribution of HMatrix $\chi^{2}$ for electrons in $Z \rightarrow e e$ events and multijet events. The solid line is for $Z \rightarrow e e$ and the dashed line is for multijet events.

To reduce such background, a loose electron is required to have a well-matched track to become a "tight electron".

To establish a match between tracks and loose electrons, tracks first are extrapolated to the EM3 layer of the calorimeter. Then a $\chi^{2}$ variable is calculated, which measures the match between a loose electron and a track according to the type of track:

- global tracks:

$$
\chi_{\text {Global }}^{2}=\left(\frac{\Delta \phi}{\sigma_{\phi, G}}\right)^{2}+\left(\frac{\Delta z}{\sigma_{z, G}}\right)^{2}+\left(\frac{\frac{E_{T}}{P_{T}}-1}{\sigma_{\frac{E_{T}}{p_{T}}, G}}\right)^{2}
$$


- Axial-only tracks:

$$
\chi_{\text {Axial }}^{2}=\left(\frac{\Delta \phi}{\sigma_{\phi, A}}\right)^{2}+\left(\frac{\frac{E_{T}}{P_{T}}-1}{\sigma_{\frac{E_{T}}{p_{T}}, A}}\right)^{2}
$$

- SMT-only tracks:

$$
\chi_{S M T}^{2}=\left(\frac{\Delta \phi}{\sigma_{\phi, S}}\right)^{2}+\left(\frac{\Delta z}{\sigma_{z, S}}\right)^{2}
$$

where $\Delta \mathrm{z}$ is the difference in the $\mathrm{z}$ position, $\Delta \phi$ is the difference in transverse direction, and $E_{T} / p_{T}$ is the ratio between transverse energy and momentum. $\sigma_{\phi}, \sigma_{z}$, and $\sigma_{\frac{E_{T}}{p_{T}}}$ are the rms values of the experimental distributions for the 3 types of tracks (denoted as G, A and S). The measured values from data are [37]:

$$
\begin{aligned}
& \sigma_{\phi, G}=6 \mathrm{mrad}, \sigma_{z, G}=0.7 \mathrm{~cm}, \text { and } \sigma_{\frac{E_{T}}{p_{T}}, G}=0.21 \\
& \sigma_{\phi, A}=8 \mathrm{mrad}, \sigma_{\frac{E_{T}}{p_{T}}, A}=0.21 \\
& \sigma_{\phi, S}=8 \mathrm{mrad}, \sigma_{z, S}=0.8 \mathrm{~cm}
\end{aligned}
$$

A $\chi^{2}$ probability $P\left(\chi^{2}, N_{\text {d.o.f. }}\right)$ is calculated for global tracks $\left(N_{\text {d.o.f. }}=3\right)$, and for axial-only and SMT-only tracks $\left(N_{\text {d.o.f. }}=2\right)$. Figure 3.6 shows the $\chi^{2}$ probability distribution in the RunII $Z \rightarrow e e$ data sample. In our analysis, the $\chi^{2}$ probability must be greater than 0.01 in order to establish a match between a loose electron and a track. When a match is established, the electron's charge is determined by the charge of the matching track. 


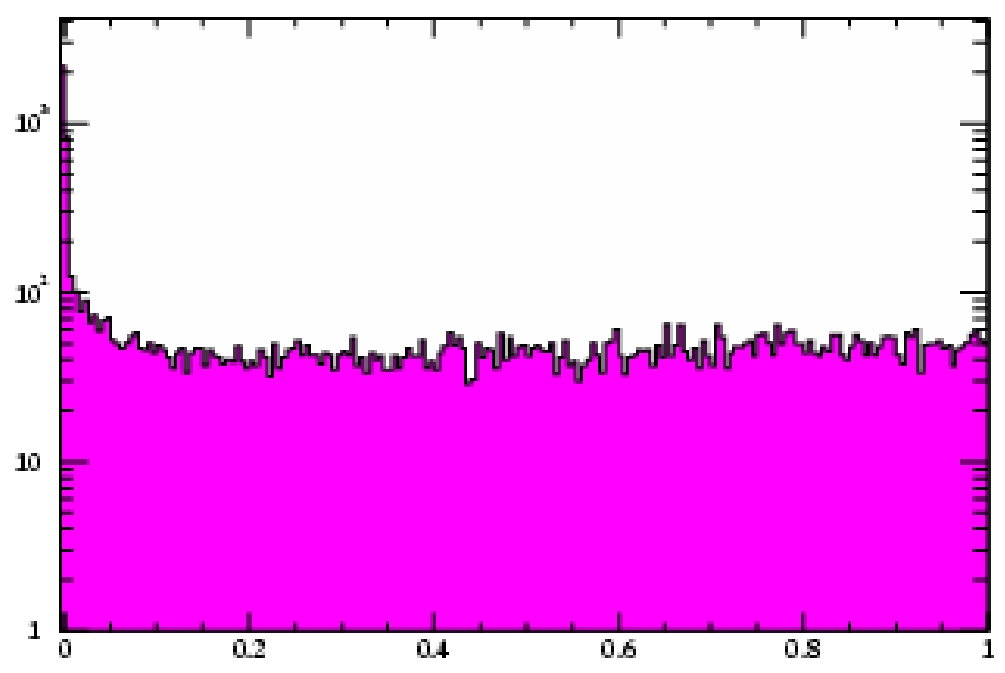

Figure 3.6: The $\chi^{2}$ probability distributions in the Run II Z data sample. The $\chi^{2}$ probability must be greater than 0.01 in order to establish a match between a loose electron and a track.

\subsection{Missing $E_{T}$}

Since neutrinos and the LSP's are neutral and weakly interacting, they do not interact in the detector and their energy is not measured. However, their presence can be inferred by the resulting momentum imbalance in the measured event. The transverse energy components of all the particles in the event are added vectorially and any significant deviation from zero is attributed to one or more particles which escaped detection. The $\#_{T}$ is defined as

$$
E_{T}=\sqrt{\left(E_{x}\right)^{2}+\left(E_{y}\right)^{2}}
$$

where

$$
E_{x}=-\sum_{i=1}^{n} E_{i} \sin \theta_{i} \cos \phi_{i}
$$


and

$$
E_{y}=-\sum_{i=1}^{n} E_{i} \sin \theta_{i} \sin \phi_{i}
$$

where $E_{i}$ is the energy deposited in cell $i$ in calorimeter.

In practice, the transverse energies of all calorimeter cells with transverse energy greater than $100 \mathrm{MeV}$ are added vectorially except for the coarse hadronic cells. Because of the large noise observed in the coarse hadronic calorimeter layers, only coarse hadronic cells belonging to a reconstructed jet are used. The vector opposite to the resulting vector sum is the raw missing energy vector and its modulus is the raw missing transverse energy $\left(\mathbb{E}_{T, \text { raw }}\right)$.

Another complication in the measurement of $\mathbb{E}_{T}$ is that the calorimeter responds differently to energy deposited by electromagnetic or hadronic particles. In events with both electromagnetic objects and jets, this response difference translates directly into missing transverse energy. In order to correct $\mathbb{E}_{T, \text { raw }}$ for this effect, a set of jet energy scale correction coefficients [38] is used. The corrected $\mathbb{E}_{T}$ is called the calorimeter missing transverse energy $\left(E_{T, C A L}\right)$ and we use this variable in our offline event selection.

\subsection{Muon Reconstruction}

The offline muon identification is based on a match between a charged particle detected in the central tracking system and a signal in the muon system. To be used as a seed for a muon object, a charged particle is required to have $p_{T}>1.5 \mathrm{GeV} / \mathrm{c}$. The signal detected in the muon system is used to reconstruct track segments both before and after the toroid magnet. The 
segments are matched together to form local muon tracks and the momentum is determined from the bend of the track passing through the magnet iron. Finally, local muon tracks are combined with the information from the central tracking system and the calorimeter to build muon objects. 


\section{Chapter 4}

\section{Data Sample, Event Generators, and Detector Simulations}

The search for chargino and neutralino pair production is based on the $\mathrm{p} \overline{\mathrm{p}}$ collision data collected with the $\mathrm{D} \varnothing$ detector. In the search, Monte Carlo simulations also play an important role. In this chapter, we describe the $\mathrm{D} \varnothing$ RunII data sample and the Monte Carlo simulation used for this analysis.

\subsection{Data Sample}

The data sample used in the analysis was collected with the D $\varnothing$ detector from September 2002 to June 2003. The luminosity delivered by the accelerator and recorded at DØ during the year 2002 and 2003 is shown in Figure 4.1. Bad runs declared by the Jet/Missing $E_{T}$ group [39] are excluded from the analysis. Since the total integrated luminosity is an important parameter for this analysis, data with bad luminosity blocks [40] are discarded. The resulting data sample has an integrated luminosity of $124.5 \mathrm{pb}^{-1}$, with an uncertainty of 
$\pm 10 \%$ determined by the performance of the DØ luminosity monitor. The latter uncertainty is expected to decrease in the future to $6 \%$ with a recalibration of the luminosity detector.

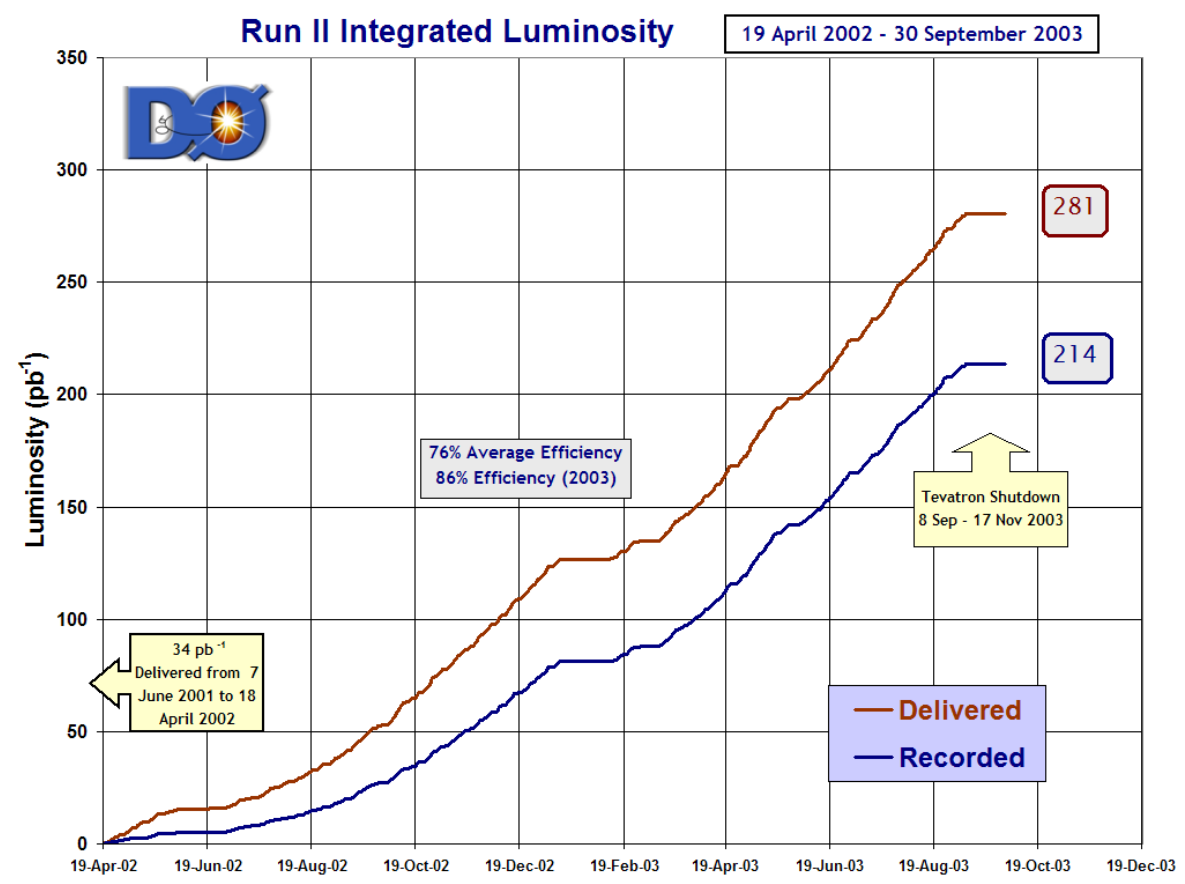

Figure 4.1: Delivered and recorded luminosity at DØ in RunII during the year 2002 and 2003.

\subsubsection{Online Triggering}

The triggers used for the signal events are single EM triggers and diEM triggers. Single EM triggers include unprescaled (EM_HI, EM_HI_SH, EM_HI_SH_TR, EM_HI_TR) and (EM_MX, EM_MX_SH, EM_MX_SH_TR, EM_MX_TR). The di-EM triggers include prescaled 2EM_2MD7 and 2EM_ 
MD12_CEM10 triggers, and unprescaled 2EM_2MD12 triggers.

The EM_HI triggers require at level 1 one EM tower with $E_{T}>10 \mathrm{GeV}$ (termed CEM(1,10)) whereas the EM_MX triggers require one EM tower with $E_{T}>15 \mathrm{GeV}(\mathrm{CEM}(1,15))$. At Level 2, all the triggers require one EM tower with $E_{T}>12 \mathrm{GeV}$. At Level 3, the EM_HI and EM_MX triggers require one electrons with $E_{T}>30 \mathrm{GeV}$. For triggers with an additional shower shape requirement for the EM cluster (EM_HI_SH and EM_MX_SH) the $E_{T}$ cut is lowered to $20 \mathrm{GeV}$. The requirement of an extra track with $P_{T}>12 \mathrm{GeV}$ at Level 3 allows for a further decrease of the $E_{T}$ cut to $12 \mathrm{GeV}$ (EM_HI_SH_TR and EM_MX_SH_TR).

The 2EM_2MD7 trigger requires two EM towers with $E_{T}>5 \mathrm{GeV}$ at level 1; two EM towers, one $E_{T}>6 \mathrm{GeV}$ and the other $E_{T}>8 \mathrm{GeV}$ at level 2; and two electrons with $E_{T}>7 \mathrm{GeV}$ at level 3. The 2EM_MD12_CEM10 trigger has a higher $E_{T}$ threshold for the leading EM tower, requiring the leading EM tower's $E_{T}>8 \mathrm{GeV}$ at level 2, and $E_{T}>12 \mathrm{GeV}$ at level 3. The 2EM_2MD12 trigger is not prescaled. It has same $E_{T}$ requirement as 2EM_2MD7 except that it requires 2 electrons with $E_{T}>12 \mathrm{GeV}$ at level 3. The details of each trigger are listed in the Table 4.1.

\subsubsection{Trigger Efficiency}

Ideally, to measure the trigger efficiency, one needs a data sample unbiased by the trigger and one measures the fraction of reconstructed objects in the sample that would have passed the trigger under studies. For example, to measure the trigger efficiency of single and di-EM triggers for di-electron events, 


\begin{tabular}{|c|c|c|c|c|}
\hline Trigger & L1 trigger & L2 trigger & L3 filter & Prescaled \\
\hline EM_HI & $\operatorname{CEM}(1,10)$ & $\mathrm{em}(1,12)$ & ele $(1,30)$ & Not \\
\hline EM_HI_SH & $\operatorname{CEM}(1,10)$ & $\operatorname{em}(1,12)$ & $\operatorname{ele}(1,20, \mathrm{sh})$ & Not \\
\hline EM_HI_SH_TR & $\operatorname{CEM}(1,10)$ & $\mathrm{em}(1,12)$ & ele $(1,12, \operatorname{sh}) \operatorname{trk}(1,12)$ & Not \\
\hline EM_HI_TR & $\operatorname{CEM}(1,10)$ & $\mathrm{em}(1,12)$ & $\operatorname{trk}(1,25)$ & Not \\
\hline EM_MX & $\operatorname{CEM}(1,15)$ & $\mathrm{em}(1,12)$ & ele $(1,30)$ & Not \\
\hline EM_MX_SH & $\operatorname{CEM}(1,15)$ & $\mathrm{em}(1,12)$ & ele $(1,20, \mathrm{sh})$ & Not \\
\hline EM_MX_SH_TR & $\operatorname{CEM}(1,15)$ & $\mathrm{em}(1,12)$ & ele $(1,12, \operatorname{sh}) \operatorname{trk}(1,12)$ & Not \\
\hline EM_MX_TR & $\operatorname{CEM}(1,15)$ & $\mathrm{em}(1,12)$ & $\operatorname{trk}(1,25)$ & Not \\
\hline 2EM_2MD7 & $\operatorname{CEM}(2,5)$ & $\mathrm{em}(2,6) \mathrm{em}(1,8)$ & $\operatorname{ele}(2,7)$ & Yes \\
\hline 2EM_MD12_CEM10 & $\operatorname{CEM}(2,5) \operatorname{CEM}(1,10)$ & $\mathrm{em}(2,6) \mathrm{em}(1,8)$ & ele $(2,7) \operatorname{ele}(1,12)$ & Yes \\
\hline 2EM_2MD12 & $\operatorname{CEM}(2,5)$ & $\mathrm{em}(2,6) \mathrm{em}(1,8)$ & $\operatorname{ele}(2,12)$ & Not \\
\hline
\end{tabular}

Table 4.1: Single and Di-EM triggers used in this analysis. The details of each trigger term are explained in the text. 
one may select muon triggered events that has di-electron events similar to the di-electron data sample we want to study, and in there measure the fraction of di-electron events that pass those EM triggers. However, given the small number of di-electron events available in such an unbiased trigger sample, we estimate the electron trigger efficiency by comparing the number of low-mass Drell-Yan events $\left(20<m_{e e}<75 \mathrm{GeV} / \mathrm{c}^{2}\right)$ found in data and predicted by MC [41]. Figure 4.2 shows the ratio of the number of low mass Drell-Yan events in data and $\mathrm{MC}$ as a function of the primary electron $E_{T}$. Figure 4.3 shows the same distribution versus the secondary electron $E_{T}$. From these two figures, it is shown that the combination of all the above single and di-electron triggers does not introduce a trigger bias if the electron pair is required to have $E_{T}^{p r i}>15 \mathrm{GeV}$ and $E_{T}^{\text {sec }}>10 \mathrm{GeV}$. Therefore, the trigger efficiency is consistent with $100 \%$.

\subsection{Monte Carlo Simulation}

Monte Carlo simulation is useful in many aspects of this analysis. For example, we completely rely on MC to study the chargino and neutralino signals. We also use $\mathrm{MC}$ in modeling some of the backgrounds that contaminate the data sample. In general, Monte Carlo simulations proceed in 2 steps: event generation and detector simulation. In the following, we will discuss these briefly. 


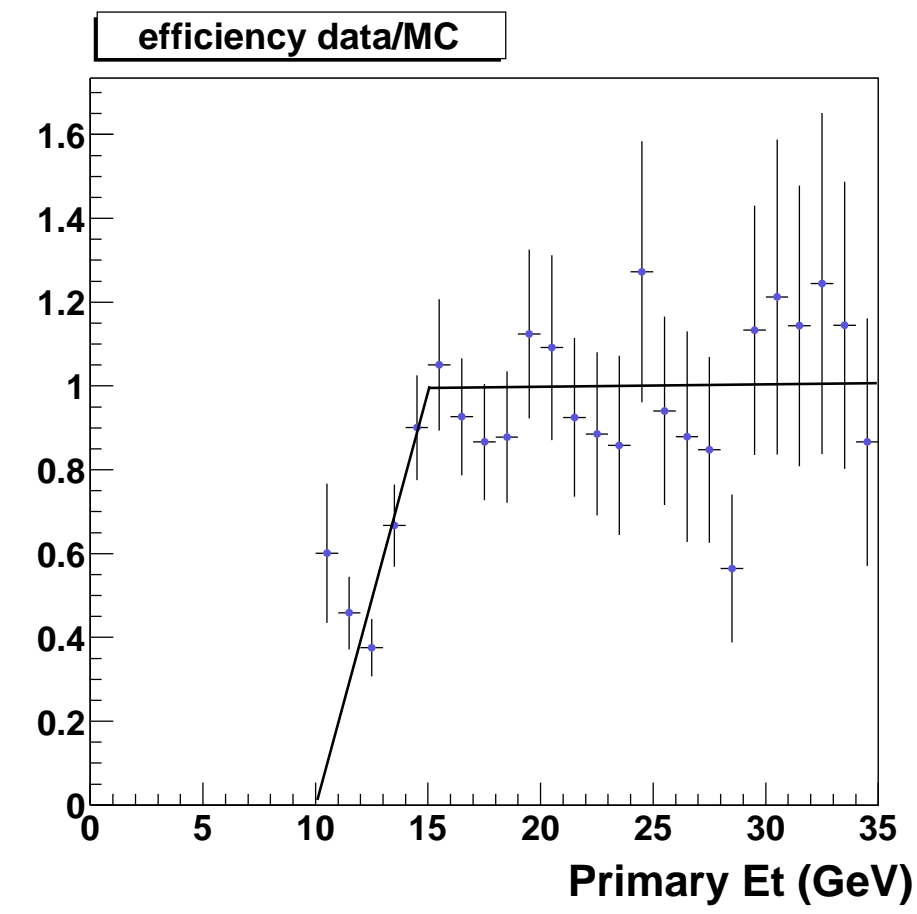

Figure 4.2: Ratio between the number of low mass Drell-Yan events in data and in $\mathrm{MC}$ as a function of the primary electron $E_{T}$. 


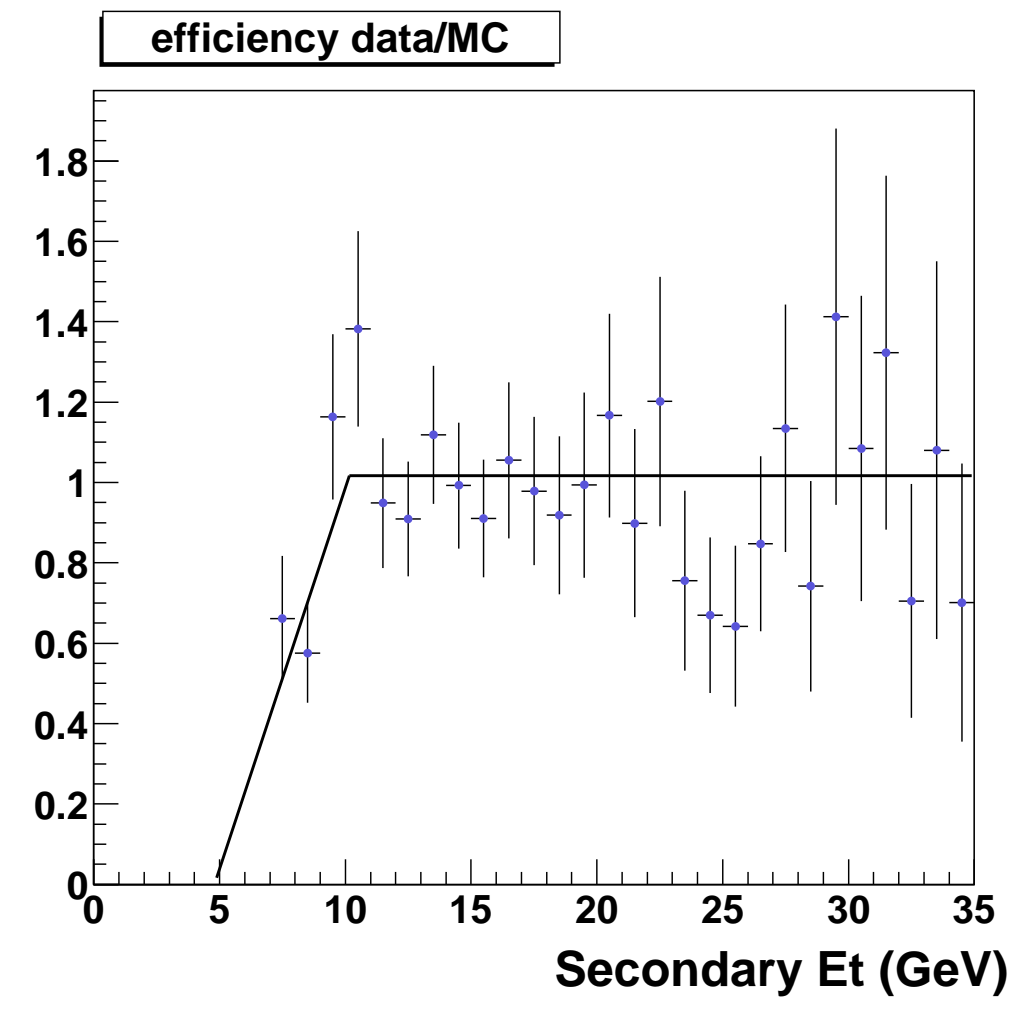

Figure 4.3: Ratio between the number of low mass Drell-Yan events in data and in MC as a function of the secondary electron $E_{T}$. 


\subsubsection{Event Generation}

Monte Carlo event generators constitute an extremely important and commonly used class of tools utilized by high energy particle physicists. The term "Monte Carlo" refers to numerical simulations of processes that may be characterized by sets of random numbers. The basic variables describing the simulated process assume values in accordance with appropriately prescribed probability distributions.

At DØ, the most commonly used Monte Carlo event generators for hadronhadron collisions are ISAJET [42], PYTHIA [43], and HERWIG [44]. For this analysis, ISAJET and PYTHIA have been used for the generation of signal events and various background processes. In practice, a user supplies the event generator with an input "card file" [45] which specifies the details of the physics processes to be simulated and the event generator outputs a list of vertices and particle types/four-vectors that come out of those vertices.

Typically, Monte Carlo event generators are the intellectual property of the theoretical physicists who developed the model which is implemented in the program. However, each of these generators follows the same basic approach in modeling high energy hadron-hadron collisions and only differs in the details of their implementation. The basic approach is:

- A primary hard scattering is generated using QCD cross-section for $2 \rightarrow 2$ or $2 \rightarrow 3$ parton processes and for appropriate parton structure functions.

- All partons participating in the hard scattering, incoming or outgoing 
for the process above, are evolved through repeated branchings according to the Altarelli-Parisi splitting functions. Through this procedure both initial and final radiations are generated.

- Quarks and gluons produced in the first two steps are then fragmented into hadronic final states. This process is known as fragmentation or hadronization. As this can not be done in perturbative QCD, different event generators employ different empirical schemes for hadronization, e.g., ISAJET uses Feynman-Field scheme [46], and PYTHIA uses Lund String fragmentation scheme [47].

- The final step in the event generation is to evolve and hadronise the leftover partons known as "spectators". There is no unique way of dealing with the leftover partons. PYTHIA uses an extension of the Lund Color scheme whereas ISAJET overlays minimum bias events on top of the primary hard scattering event.

\subsubsection{Detector Simulation}

The second step in the full simulation is to compute the detector response to the simulated events. The full detector simulation at D $\varnothing$ consists of two programs: DØgstar [48] and DØSim [49].

The DØgstar program is based on the GEANT [50] program developed at CERN. GEANT is used to describe the true geometry of a detector by building it from a library of material volumes of known shapes. It also has extensive knowledge of the interactions between particles and matter. It takes tracks 
from the event generator and propagates them through the detector volumes, at each step checking the probability of interaction between the particle and the local material. If a particle is deemed to interact in the detector material and produces new particles, those particles are propagated through the detector in turn. If an electromagnetic shower starts in material, GEANT will follow the produced daughters through each stage of the shower, depositing the energy loss of the shower in the successive detector cells. The ultimate output of this simulation is typically a list of lost energy and/or arrival times for hits in individual detector cells.

The $D \emptyset$ Sim program takes the output from DØGSTAR and does the digitization for each sub-detector, pileup, overlaps with additional minbias events and adds electronics noise and hit resolution smearing. The output MC events are in the exact same format as the data collected by the $\mathrm{D} \varnothing$ detector and are reconstructed by DØReco.

\subsubsection{Corrections to the Monte Carlo Simulations}

The simulated Monte Carlo events suffer from several deficiencies that prevent them from being a truly accurate representation of the real data. It has been found that several refinements to the calorimeter simulations are required in order to simulate the data in a more realistic fashion. These refinements center mainly on various (additional) sources of noise that are under-estimated in the simulation. Such noise sources are mainly the uranium current in the calorimeter, the electronics noise, and fluctuations in the underlying events.

We adopt the following simple model to estimate the correct MC electron 
energy $\left(E_{M C}^{c o r r}\right)$ from the reconstructed MC electron energy $\left(E_{M C}^{r e c o}\right)$ :

$$
E^{\prime}=\alpha E_{M C}^{r e c o}+\beta
$$

and

$$
E_{M C}^{c o r r}=E^{\prime}+\operatorname{Gauss}\left(\sigma, E^{\prime}\right)
$$

where in (4.1) $\alpha$ is the EM energy scale, $\beta$ is the EM energy offset, and in (4.2) $\sigma$ is the additional noise smearing term and $\operatorname{Gauss}\left(\sigma, E^{\prime}\right)$ represents a random number get from a Gaussian distribution that has a center value of $E^{\prime}$ and $\mathrm{rms}$ of $\sigma$. By comparing data and MC, these empirical variables are determined to be: $\alpha=1.0, \beta=0.0 \mathrm{GeV}, \sigma=0.047$ for electrons in the central detector region [51].

The under-estimated noise in MC also needs to be corrected in the simulation of $\not_{T}$. Studies [41] shown that MC $E_{T}$ needs to be smeared by a Gaussian with $\sigma=0.30 \#_{T}$ and an additional Gaussian of $\sigma=1.5 \mathrm{GeV}$ in order to describe data satisfactorily. 


\section{Chapter 5}

\section{Data Analysis and Background Studies}

A search for new particles in data usually takes three steps: the enrichment of the data sample in signal events relative to backgrounds, the estimation of the expected number of background events, and the estimation of the signal expected from a model of the new physics for which one is searching. In this chapter, we describe the data reduction and background estimate for our chargino and neutralino search in the like-sign di-electron channel.

\subsection{Offline Event Selection}

The standard method physicists use to select events is through the introduction of a series of selection requirements called cuts. The purpose of these cuts is to preserve the greatest amount of signal while rejecting most of the background. By comparing a representative mSUGRA mass point $\left(m_{0}=100\right.$ $\mathrm{GeV} / \mathrm{c}^{2}, m_{1 / 2}=100 \mathrm{GeV} / \mathrm{c}^{2}, \tan (\beta)=2.5, A_{0}=0$, and $\left.\operatorname{sign}(\mu)=-1\right)$ to various sources of background, we developed a set of selection cuts for offline event selection. They are described as follows: 


\subsubsection{Electron ID Cuts}

The most important cut in this analysis is the requirement of a like-sign electron pair. Accurate measurement of the charge of electrons requires electrons pass through the whole geometric volume of the tracking system. Since only the central region of the detector is fully covered by the tracking system, we consider central electrons with $|\eta|<1.1$. The electron ID cuts described in chapter 3 are used for defining good and isolated central electrons. To recapitulate:

- EM fraction $\left(f_{E M}>0.90\right)$

- Isolation fraction $\left(f_{\text {iso }}<0.15\right)$

- Cluster shape $\left(\chi^{2}<20\right)$

These cuts are well established for electron signals and standard within DØ. The efficiency of these cuts is determined using a clean $\mathrm{Z} \rightarrow e e$ sample. The details are described in Appendix A. The electron identification efficiencies in data and MC calculated by this method are $85.0 \pm 2.0 \%$ and $96.6 \pm 2.0 \%$ respectively. This discrepancy is mainly caused by the imperfection in the GEANT simulation of the calorimeter and we apply an additional correction factor to MC samples to compensate the discrepancy. Since there are no obvious $\eta$ dependences observed for the correction factor for electrons in the $\mathrm{CC}$ region [52], an average correction factor of $0.88 \pm 0.03$ (relative error $3.4 \%$ ) is used. 


\subsubsection{Track Matching Requirement}

A tight electron must have an isolated track associated with it. We require the track matching $\chi^{2}$ probability between a loose electron and an isolated track be greater than 0.01 in order to establish a match, as described in Chapter 3 .

We use the $\mathrm{Z} \rightarrow$ ee sample once again to measure the tracking efficiency. The details of the measurement are described in Appendix B. The tracking efficiency measured in data is $72.4 \pm 0.5 \%$ and in $\mathrm{MC}$ is $87.4 \pm 0.4 \%$. The discrepancy between MC and data is independent of electron's $\eta$ [51], therefore a flat correction factor of $0.83 \pm 0.01$ (relative error $0.8 \%$ ) is applied to the $\mathrm{MC}$ samples.

\subsubsection{Central Di-Electron Pair}

This selection cut requires two tight central electrons with $E_{T}>15 \mathrm{GeV}$ for the leading electron and $E_{T}>10 \mathrm{GeV}$ for the secondary electron. To justify this cut, we plot the $E_{T}$ distribution of the primary and secondary electron arising from the signal and various sources of backgrounds in Figure 5.1 and Figure 5.2 respectively. It is clear that the $E_{T}$ requirement does not remove any significant amount of signal and is not effective in removing backgrounds with two true electrons. But this cut is very important for removing background with fake electrons coming from jet since the number of fake electrons drops quickly as the $E_{T}$ cut increases. In the data sample, a total of 2349 events passed the central di-electron selection cut. 

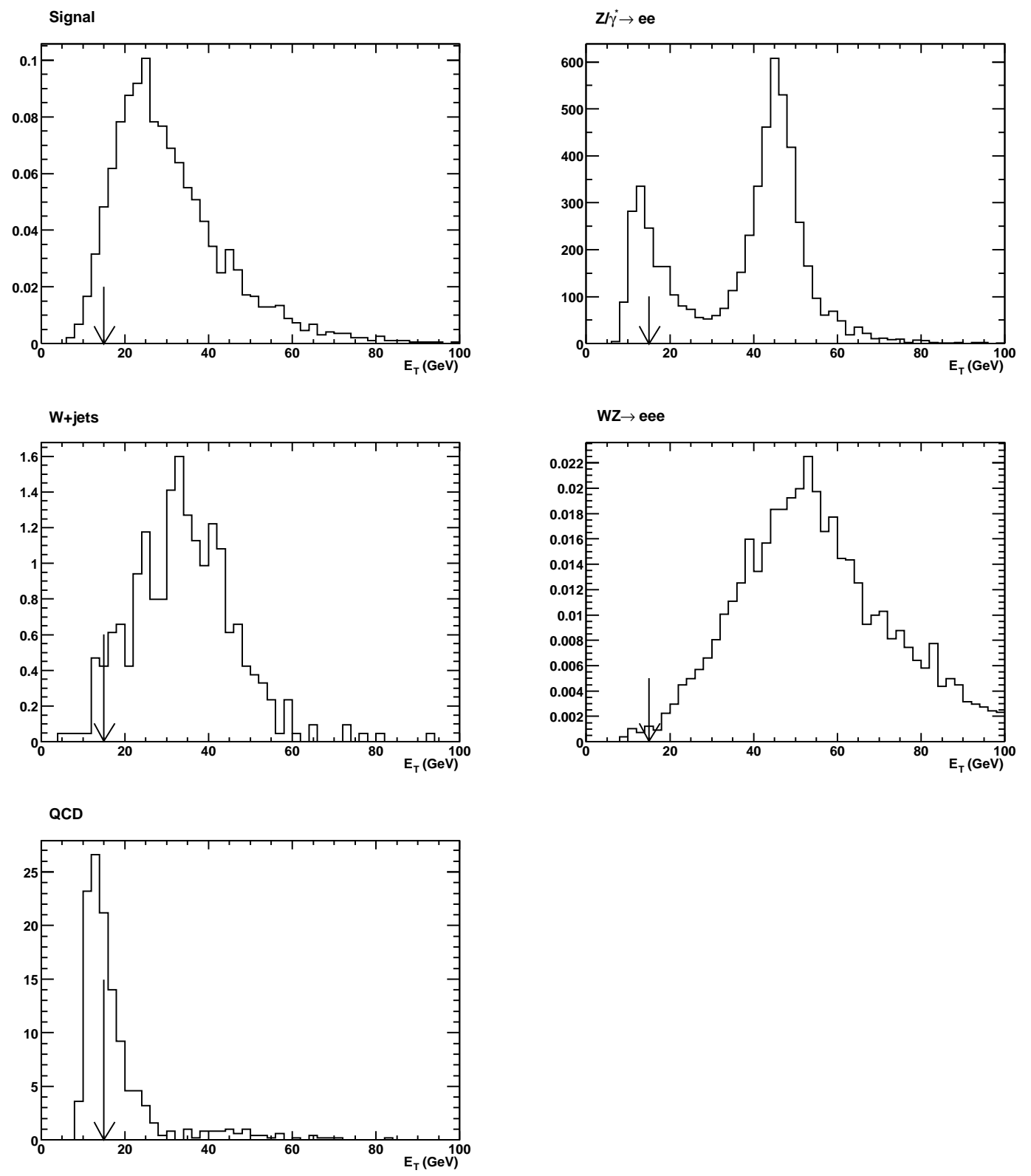

Figure 5.1: $E_{T}$ distributions of the primary electrons in signal and background. The arrows represent the chosen cut. Number of events is normalized to an integrated luminosity of $124.5 \mathrm{pb}^{-1}$. 

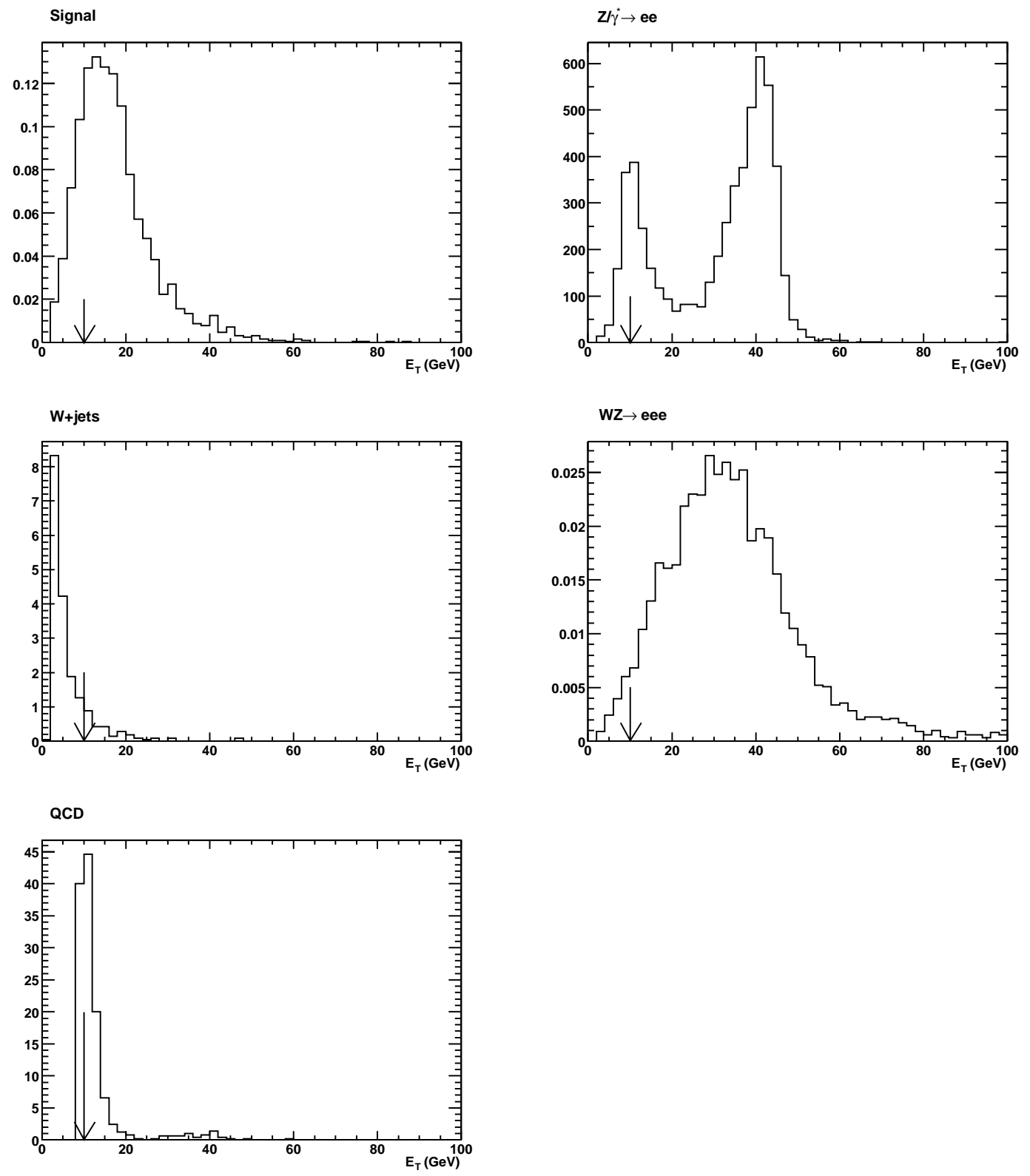

Figure 5.2: $E_{T}$ distributions of the secondary electrons in signal and background. The arrows represent the chosen cut. Number of events is normalized to an integrated luminosity of $124.5 \mathrm{pb}^{-1}$. 


\subsubsection{Like-Sign Di-Electron Pair}

In order to effectively remove Standard Model di-electron backgrounds which are mostly opposite-sign electron pair events, a like-sign cut $\left(e^{ \pm} e^{ \pm}\right)$ is required. If the electrons are like-sign, the event is saved for further analysis. If the electron pair has opposite signs, we search for a third electron with $E_{T}>10 \mathrm{GeV}$ in the event to maximize the sensitivity of the like-sign channel. When a third electron is found, it should have the same charge as either the primary electron or the secondary electron. If the matching electron has $E_{T}>$ $15 \mathrm{GeV}$, then the like-sign electron pair is saved.

In our data, a total of 44 events passed the like-sign requirement.

\subsubsection{Invariant Mass Cut}

The invariant mass of the like-sign electron pair in signal events is expected to be relatively soft in the region of the chargino mass $\left(\approx 100 \mathrm{GeV} / \mathrm{c}^{2}\right)$ we are studying. Figure 5.3 shows the predicted invariant mass for the signal and various backgrounds. We require the invariant mass of the like-sign electron pair between $20 \mathrm{GeV} / \mathrm{c}^{2}$ and $75 \mathrm{GeV} / \mathrm{c}^{2}$. This cut reduces the $Z \rightarrow e e$ background dramatically. A total of 11 events passed the invariant mass cut.

\subsubsection{Missing Transverse Energy Cut}

Due to the presence of the LSP and neutrino in the final state of $\tilde{\chi}_{1}^{ \pm} \tilde{\chi}_{2}^{0}$ decay, we expect to observe significant missing energy in the signal events. Figure 5.4 shows the transverse missing energy distribution for the signal and 

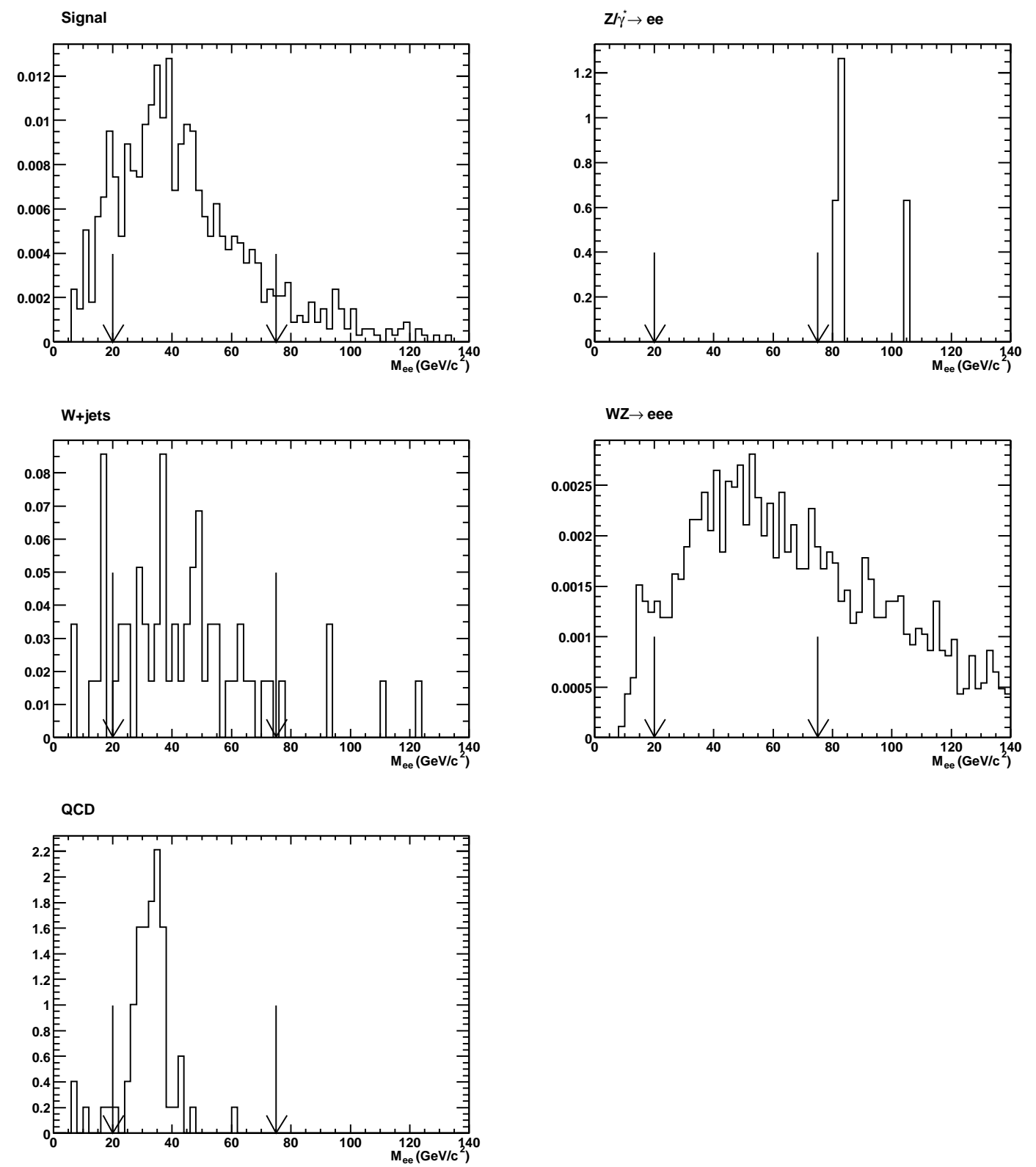

Figure 5.3: Invariant mass distribution for like-sign electron pairs in the signal and backgrounds. The arrows represent the selection: $20 \mathrm{GeV} / \mathrm{c}^{2}<M_{e e}<75$ $\mathrm{GeV} / \mathrm{c}^{2}$. 

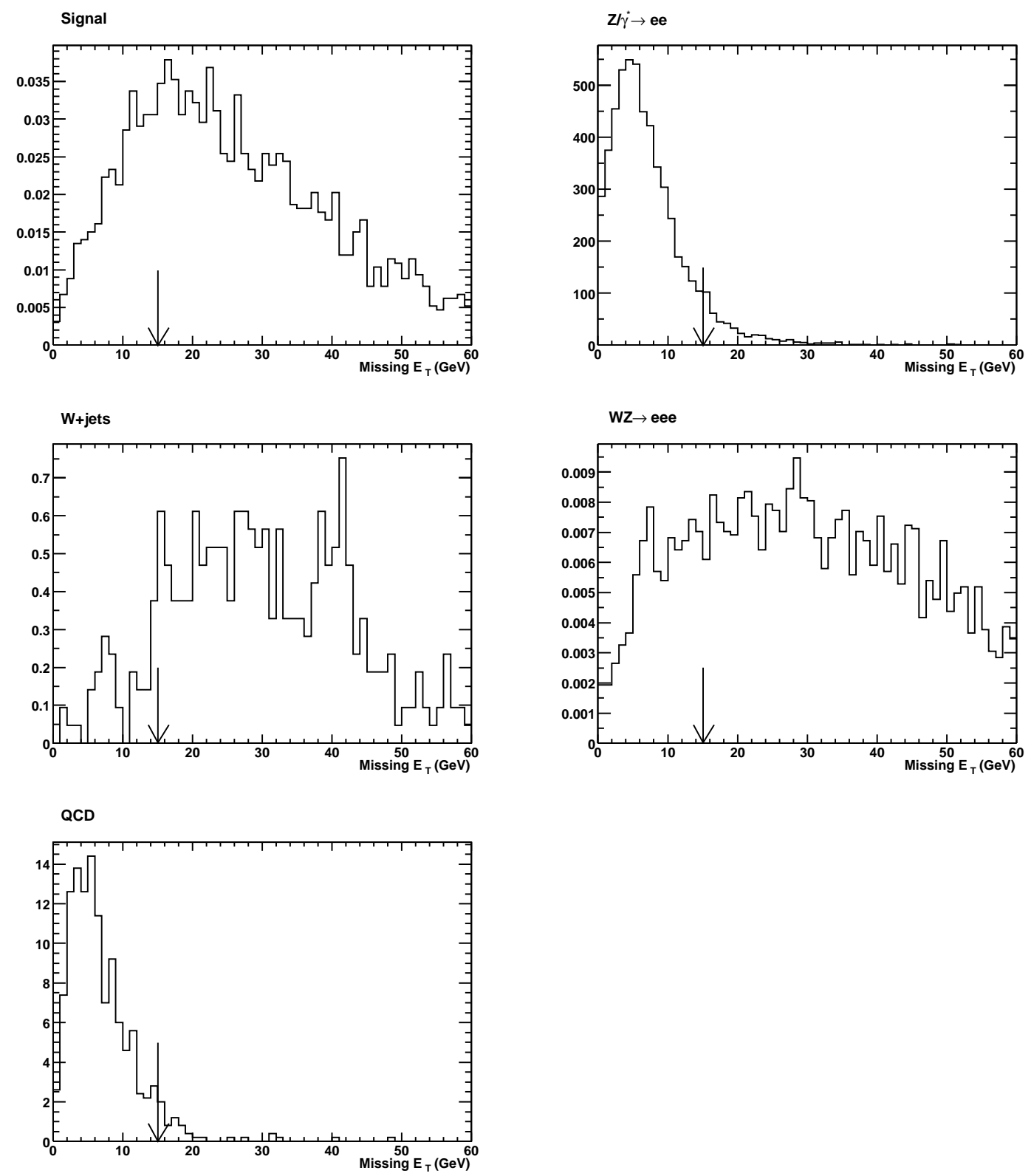

Figure 5.4: Transverse missing energy distribution for the signal and backgrounds. The arrow represents the selection: $\mathbb{E}_{T}>15 \mathrm{GeV}$. The number of events is normalized to an integrated luminosity of $124.5 \mathrm{pb}^{-1}$. 
various backgrounds after requiring 2 central tight electrons in the event. We apply a $\mathbb{E}_{T}>15 \mathrm{GeV}$ cut. This cut is effective in reducing the backgrounds from $Z / \gamma^{*} \rightarrow e e$ and QCD. After this cut, only 1 event in data survives.

To summarize, the cumulative number of data events after each cut in this analysis is shown in Table 5.1.

\begin{tabular}{|c|c|}
\hline \hline Cut & Num of events \\
\hline Central Di-Electron with $p_{T}^{p r i}>15 \mathrm{GeV}$ and $p_{T}^{s e c}>10 \mathrm{GeV}$ & 2349 \\
Like-Sign Electron Pair & 44 \\
$20 \mathrm{GeV}<M_{e e}<75 \mathrm{GeV}$ & 11 \\
$\mathbb{E}_{T}>15 \mathrm{GeV}$ & 1 \\
\hline \hline
\end{tabular}

Table 5.1: A summary of the successive cuts used in this analysis and the number of events passing each cut.

\subsubsection{The Candidate Event}

The side-view and end-view of the candidate event surviving all the event selection cuts are show in Figure 5.5 (a) and (b). These figures are produced by using DØVE [53], an event display software package developed at DØ.

The candidate event was taken on January 1, 2003. Its run number is 
169917 and the event number is 7189585 . Figure 5.5(a) shows the primary electron $\left(E_{T}=43.0 \mathrm{GeV}\right.$, upper right $)$ and the secondary electron $\left(E_{T}=\right.$ $11.9 \mathrm{GeV}$, lower left) in the $r-z$ plane. Figure 5.5(b) shows the primary electron(upper right) and the secondary electron(lower left) in the $r-\phi$ plane. In these figures, we see that two electrons with negative charge are found in the candidate event. In the $r-\phi$ view, the track for the secondary electron seems pointing to the neighbor tower of the electron. This is an artifact due to the coarse granularity of the calorimeter towers in $\phi$ direction implemented in DØVE. The invariant mass of the electron pair is $44.2 \mathrm{GeV} / \mathrm{c}^{2}$ and the transverse missing energy of the event is $40.1 \mathrm{GeV}$. The details of this event are shown in Table 5.2.

\subsection{Backgrounds}

After completing the analysis of the $\mathrm{D} \varnothing$ data, we found 1 event survived all our selection requirement. In order to investigate further the origin of the candidate event, we need to estimate the contribution from various physics processes as well as misidentification of electrons. In principle, any known process that could give rise to an event with like-sign electrons is considered a background. These processes can be classified as (a) Background from Standard Model processes (SM backgrounds), and (b) Instrumental backgrounds due to the misidentification of jets as electrons.

There are two methods that may be used for estimating the background: from data or Monte Carlo. We use the data to estimate backgrounds whenever 
E scale: $37 \mathrm{GeV}$

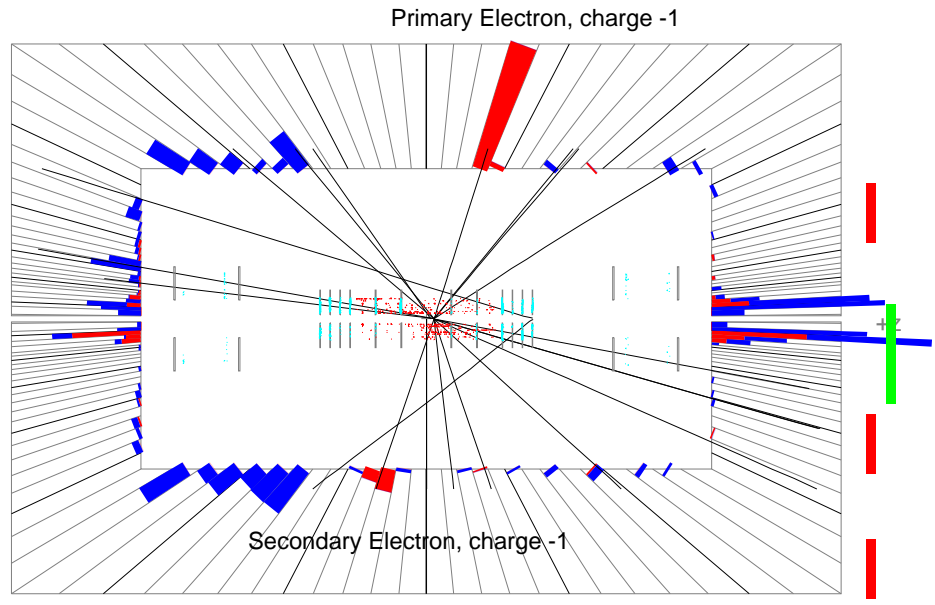

$180 \bigodot 0$

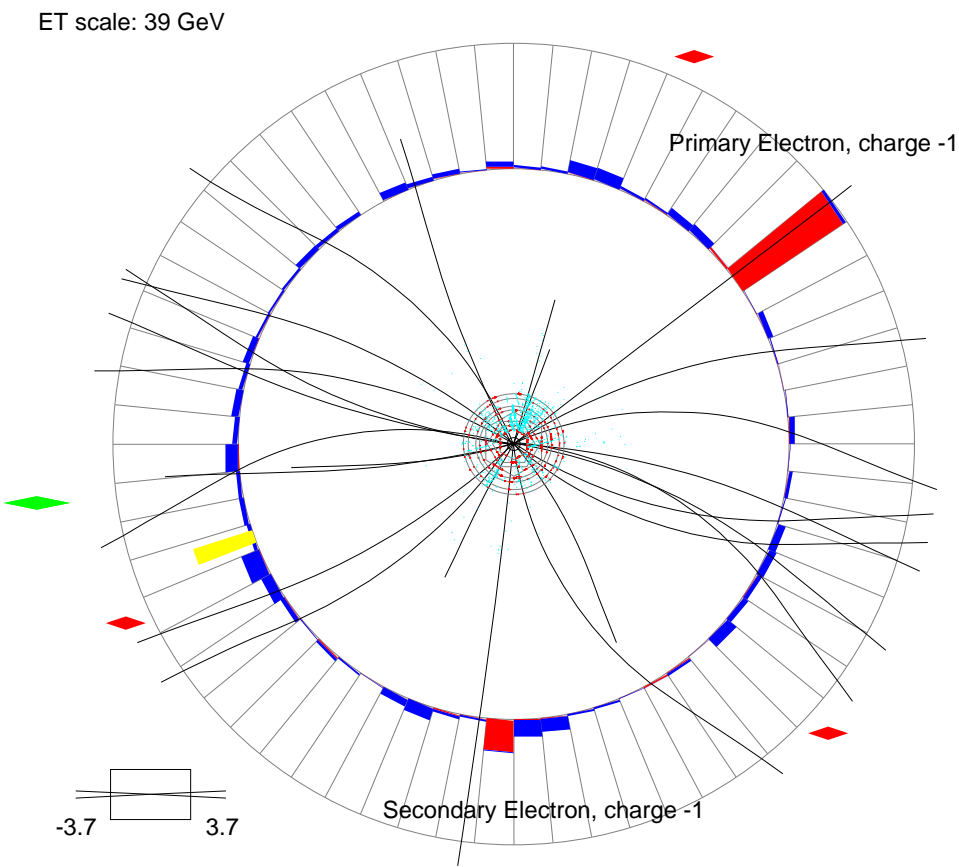

Figure 5.5: r-z (top) and r- $\phi$ (bottom) view of the candidate event 


\begin{tabular}{|c|c|c|c|c|}
\hline \multicolumn{3}{|c|}{ run num: 169917} & \multicolumn{2}{|c|}{ evt num: 7189585} \\
\hline \multicolumn{5}{|l|}{ Electrons } \\
\hline$E_{T}(\mathrm{GeV})$ & $\eta_{c a l}$ & $\phi_{c a l}$ & $f_{i s o}$ & $\chi_{H M x 8}^{2}$ \\
\hline $43.0 \pm 3.0$ & $0.312 \pm 0.015$ & $0.663 \pm 0.005$ & -0.005 & 4.2 \\
\hline $11.9 \pm 1.2$ & $-0.350 \pm 0.015$ & $4.629 \pm 0.005$ & 0.08 & 18.1 \\
\hline \multicolumn{5}{|l|}{ Tracks } \\
\hline$p_{T}(\mathrm{GeV} / \mathrm{c})$ & $\eta_{t r k}$ & $\phi_{t r k}$ & $\chi_{\text {trk-matching }}^{2}$ & charge \\
\hline 41.6 & 0.31 & 0.66 & 0.88 & -1 \\
\hline 8.3 & -0.34 & 4.61 & 0.05 & -1 \\
\hline \multicolumn{5}{|c|}{$M_{e e}=44.2 \mathrm{GeV} / \mathrm{c}^{2}$} \\
\hline \multicolumn{5}{|c|}{$E_{T}=40.1 \mathrm{GeV}$} \\
\hline
\end{tabular}

Table 5.2: The candidate event summary with run number 169917 and event number 7189585 . 
possible.

\subsubsection{SM Background}

We considered the following SM background processes and estimated their contributions from MC simulation.

\section{Z/Drell-Yan processes $\left(Z / \gamma^{*} \rightarrow e e\right)$}

The cross section for the Drell-Yan process depends strongly on the mass of $\mathrm{Z}^{*} / \gamma^{*}$, so we generate two sets of MC events with invariant mass between 20-60 GeV/c and 60-130 GeV/c . Events were not generated for invariant mass $<20 \mathrm{GeV} / c^{2}$ because electrons produced in such events would be too soft to pass the offline electron $E_{T}$ cuts. No events were generated for invariant mass $>130 \mathrm{GeV} / \mathrm{c}^{2}$ neither because we are interested in the low invariant mass events.

Since PYTHIA calculates only leading order(LO) cross section, the PYTHIA cross section of Drell-Yan process is scaled by a K-factor of 1.3. This scale factor was obtained by normalizing the expected number of events to the observed number of events with all other backgrounds subtracted in Z mass region. Therefore, the corrected $\sigma\left(Z / \gamma^{*} \rightarrow e e\right)$ are $199 \mathrm{pb}$ and $237 \mathrm{pb}$ for the $\mathrm{Z} / \gamma^{*}$ mass region of $20-60 \mathrm{GeV} / \mathrm{c}^{2}$ and $60-120 \mathrm{GeV} / \mathrm{c}^{2}$ respectively. While estimating the background, these 2 sets of events are treated as independent backgrounds and the total contribution was obtained by summing them up.

In a data sample of $124.5 \mathrm{pb}^{-1}$, the total number of $\mathrm{Z} /$ Drell-Yan events contributing to the background is estimated to be $0 \pm 0.01$. 


\section{WZ process with $W \rightarrow e \nu$ and $Z \rightarrow e e$}

The cross section times branching ratio for $\mathrm{WZ} \rightarrow(e \nu)(e e)$ is $8.99 \times 10^{-3}$ pb, i.e. only few events are expected in our data sample. We apply all the offline event selection cuts to the WZ MC sample and the selection efficiency is $4.2 \pm 0.5 \%$. Therefore, the effective cross section is $0.38 \mathrm{fb}$ and we expect $0.05 \pm 0.01 \mathrm{WZ}$ events in our final data sample.

$\mathbf{W}(\rightarrow e+\nu)+\gamma$

We generate $\mathrm{W} \gamma$ events using PYTHIA and only pass events with photon energy greater than $10 \mathrm{GeV}$ through the full detector simulation. The corresponding effective cross section is $11.8 \mathrm{pb}$, which includes the effect of the photon energy cut. The offline selection efficiency is $(4.5 \pm 2.7) \times 10^{-5}$. This reduces the cross section after selections for $\mathrm{W}(\rightarrow e+\nu)+\gamma$ to $0.54 \mathrm{fb}$. We estimate that there should be $0.07 \pm 0.04 \mathrm{~W} \gamma$ events in the data sample.

In summary, the total numbers of MC events and their cross sections for the simulated SM backgrounds are listed in Table 5.3.

\subsubsection{Instrumental background}

The instrumental background arises mainly from misidentification of jets as "loose electrons", i.e. a validated EM calorimeter cluster, which is caused by upward fluctuations in the ratio of the electromagnetic versus the hadronic component in the energy deposition pattern of jets. When a fake loose electron in an event is matched with a nearby track, the event could may appear as a 


\begin{tabular}{|l|c|c|}
\hline \hline Process & $\sigma \times$ B.R.(pb) & Num of MC Evts \\
\hline \hline $\mathrm{Z} / \gamma^{*} \rightarrow e e\left(20<m_{e e}<60 \mathrm{GeV} / \mathrm{c}^{2}\right)$ & 199 & 25,000 \\
$\mathrm{Z} / \gamma^{*} \rightarrow e e\left(60<m_{e e}<130 \mathrm{GeV} / \mathrm{c}^{2}\right)$ & 237 & 24,750 \\
$\mathrm{WZ} \rightarrow(e \nu)(e e)$ & $8.99 \times 10^{-3}$ & 11,000 \\
& & \\
$W(\rightarrow e \nu)+\gamma\left(E_{\gamma}>10 \mathrm{GeV}\right)$ & 11.8 & 35,000 \\
\hline \hline
\end{tabular}

Table 5.3: The cross sections and number of MC events generated for the SM backgrounds.

like-sign di-electron event In our analysis, the main instrumental backgrounds are from the QCD and $\mathrm{W}+$ jets processes. We estimate the QCD background from data and the $\mathrm{W}+$ jet background from both MC and data.

\section{QCD background}

To select QCD events from data, we invert the standard EM id cuts by requiring the EM object's $\chi_{H M x}^{2}>25$ instead of $\chi_{H M x}^{2}<20$. Since the cross section for QCD jet production is several orders of magnitude larger than the processes producing real electrons, and because fake electrons tend to have large $\chi_{H M x}^{2}$ values, this selected sample is dominated by QCD background events. If we assume that the properties of the QCD background do not change with the $\chi^{2}$ cut, we can use the large QCD sample with $\chi_{H M x}^{2}>25$ 
to represent the much smaller QCD sample with $\chi_{H M x}^{2}<20$. For the sake of convenience, we name the QCD sample with $\chi^{2}<20$ the HMx20 sample and the sample with $\chi^{2}>25$ the HMx25 sample.

We then define "the QCD scale factor", between the HMx20 sample and the HMx25 sample from data as:

$$
f_{Q C D}=\frac{N_{\chi^{2}<20}}{N_{\chi^{2}>25}}
$$

where $N_{\chi^{2}<20}$ is the number of QCD background events with 2 EM objects both passing $\chi_{H M x}^{2}<20$ cut and $N_{\chi^{2}>25}$ is the number of QCD events with 2 EM objects both passing the $\chi_{H M x}^{2}>25$ cut. Following the above assumption, this ratio should not change when we apply additional event selection cuts to both samples.

To estimate the QCD scale factor, we select events with 2 central EM objects of $\chi^{2}>25, E_{T}>15 \mathrm{GeV}$ and $10 \mathrm{GeV}$, where one of the EM objects matches with a track. This produces the HMx25 QCD sample. We also select events with 2 central EM objects passing the standard EM id cuts, $E_{T}>15$ $\mathrm{GeV}$ and $10 \mathrm{GeV}$, where one of the EM objects has a track match. In this latter sample, we remove the contributions from all known SM processes that produce two real electrons. The resulting sample is the HMx20 QCD sample.

In Figure 5.6, we plot the invariant mass distribution for the HMx25 QCD sample (top curve) and the HMx20 QCD sample (bottom curve). The distributions agree well in shape, which confirms our assumption that the HMx cut is uncorrelated with the other properties of the sample and that the $\mathrm{HMx} 20$ sample can be estimated from the HMx25 sample. We then count the number 
of events with invariant mass between $25 \mathrm{GeV} / \mathrm{c}^{2}$ and $75 \mathrm{GeV} / \mathrm{c}^{2}$ and estimate the QCD scale factor to be $0.201 \pm 0.003$ (relative error $1.5 \%$ ).

After applying all other offline event selection cuts to the HMx25 QCD sample, we are left with 4 like-sign di-electron events. By applying the QCD scale factor, we estimate that QCD process should contribute $0.80 \pm 0.40$ events in the data sample.

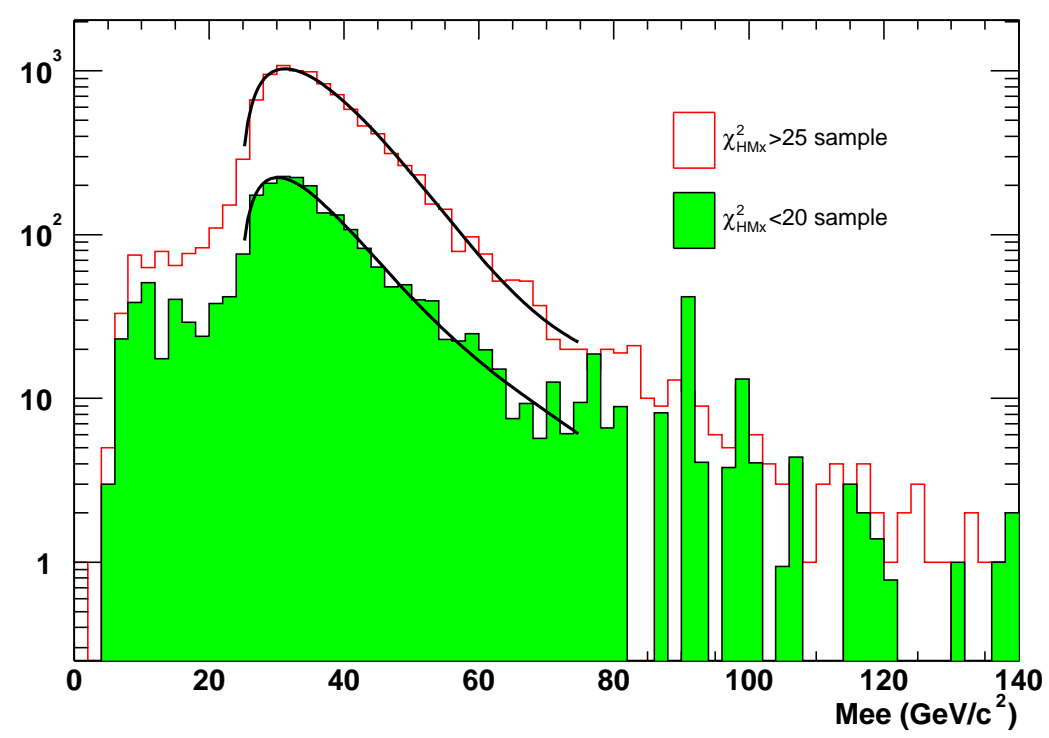

Figure 5.6: Invariant mass distributions for QCD events which pass $\chi^{2}>25$ cut (top curve) and $\chi^{2}<20$ cut (bottom solid histogram). There is only 1 EM object with a track match in these events. 


\section{$\mathrm{W}+$ jets background}

If a jet from the $\mathrm{W}+$ jets process is misidentified as a loose electron and matched with a nearby track, the event has $50 \%$ chance to appear as a like-sign di-electron event. To estimate this source of background, we used both a MC sample of 44,000 $\mathrm{W}+$ jets events as well as the data sample itself. When a jet passes the standard EM ID cuts, we call it an EM-like jet. To estimate the background from $\mathrm{W}+$ jets process, we look for EM-like jets in MC events and then apply the fake probability of track matching.

To estimate the probability for an EM-like jet to have a track match, we use events that have two jets and a loose electron [51]. We also require that the loose electron to be coininciding with one of the jets and back-to-back with the other jet so the event selected is actually a di-jet event with one jet reconstructed as a fake loose electron. The probability of an accidental track match for an EM-like jet equals then the fraction of the fake loose electrons that are found to have a track match. Figure 5.7 shows the distribution of the fake track match probability as a function of detector $\eta$. Since there is no obvious $\eta$ dependence, we use the average value $2.30 \pm 0.23 \%$ with the systematic error conservatively estimated to be $10 \%$.

When passing the $\mathrm{W}+$ jets $\mathrm{MC}$ sample through the offline selection cuts, we drop the track match requirement for one of the two electrons and instead apply the fake track match rate for the EM like jet. Among the MC events passing the modified selection cuts, we expect $50 \%$ of them to be like-sign events due to the randomness of the track matching. We estimate that $\mathrm{W}+\mathrm{jets}$ process contributes $0.67 \pm 0.18$ in the final data sample. 


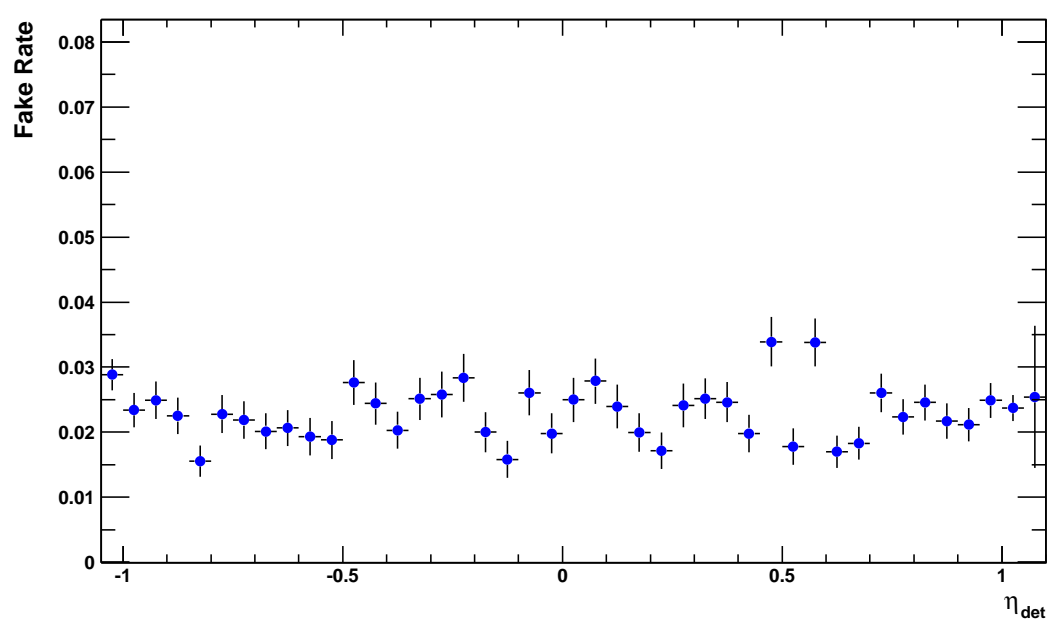

Figure 5.7: distribution of fake probability of track matching for EM like object as a function of detector $\eta$.

\subsubsection{Estimate of Uncertainties}

The following sources of statistical and systematic uncertainties have been considered in the estimation of the background contributions from various sources.

- Statistical uncertainties are caused by the finite size of event samples. If the size of the event sample is $N$, its relative statistical error is $\frac{1}{\sqrt{N}}$. The statistical uncertainty has been calculated for all the backgrounds.

- For backgrounds estimated with Monte Carlo events, one of the major uncertainties is from the integrated luminosity 10\% (Section 4.1). Other uncertainties include the uncertainties due to $\mathrm{MC} /$ data corrections. Among them, the uncertainty of the electron identification effi- 
ciency correction is $3.4 \%$ (Section 5.1.1) and that of the track matching efficiency correction is $0.8 \%$ (Section 5.1.2).

- The uncertainties of the $\mathrm{W}+$ jets background also include the uncertainty in the probability of spurious track matching (10\%) (Section 5.2.2) besides those uncertainties applicable for MC events.

- The uncertainties of QCD background include the uncertainty of the QCD scale factor 1.5\% (Section 5.2.2) but not those uncertainties applicable for MC events.

\begin{tabular}{|l|c|}
\hline \hline Source of Uncertainty & Uncertainty \\
\hline Statistical & $\frac{1}{\sqrt{N}}$ \\
Luminosity & $10 \%$ \\
Electron identification & $3.4 \%$ \\
Electron track matching & $0.8 \%$ \\
Spurious track matching (for W+jet background) & $10 \%$ \\
QCD scale factor (for QCD background) & $1.5 \%$ \\
\hline \hline
\end{tabular}

Table 5.4: The statistical and systematic uncertainties for the estimate of backgrounds. 
To summarize, we list the above uncertainties in Table 5.4. The overall uncertainty is the quadratic sum of the absolute statistical and systematic uncertainties, assuming all systematic uncertainties are uncorrelated.

\subsection{Comparison Between the Data Sample and Backgrounds}

To check the validity of the data sample and the estimate of backgrounds for this analysis, we first compare the central di-electron sample with the backgrounds. Figure 5.8 and Figure 5.9 show the invariant mass and missing $E_{T}$ distributions for the central di-electron data and backgrounds. In these figures, dots with error bars represent the data and the solid lines are sums of backgrounds, normalized to the same integrated luminosity as the data. We observed that data and the background generally match well. In Figure 5.8, one can see some excess of events around $90 \mathrm{GeV} / \mathrm{c}^{2}$ associated with the QCD background, which is caused by a few real $Z \rightarrow e e$ events with large $\chi^{2}$ being categorized as QCD events.

Table 5.5 further shows the normalized number of events passing each offline event selection cut for all the backgrounds. For comparison, we also list the number of data events that passes each cut. We observed that data and background generally match well after each selection cut. The only deviation we observed is that there are more like-sign di-electron events (immediately after selection cut 2) in data than in the backgrounds. Further examination indicates that the excess of extra like-sign events stems from large invariant 


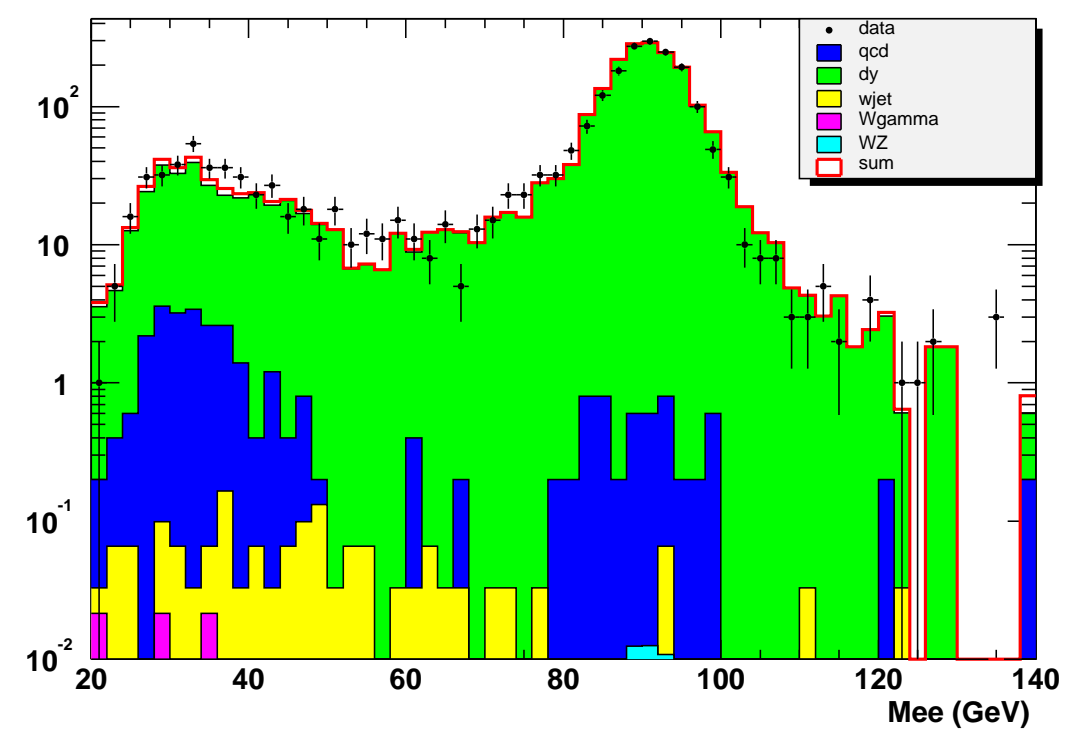

Figure 5.8: Invariant mass distributions for central di-electron sample (with 2 central tight electrons). Data (in dots with error bars) and sum of backgrounds (solid line) are consistent. 


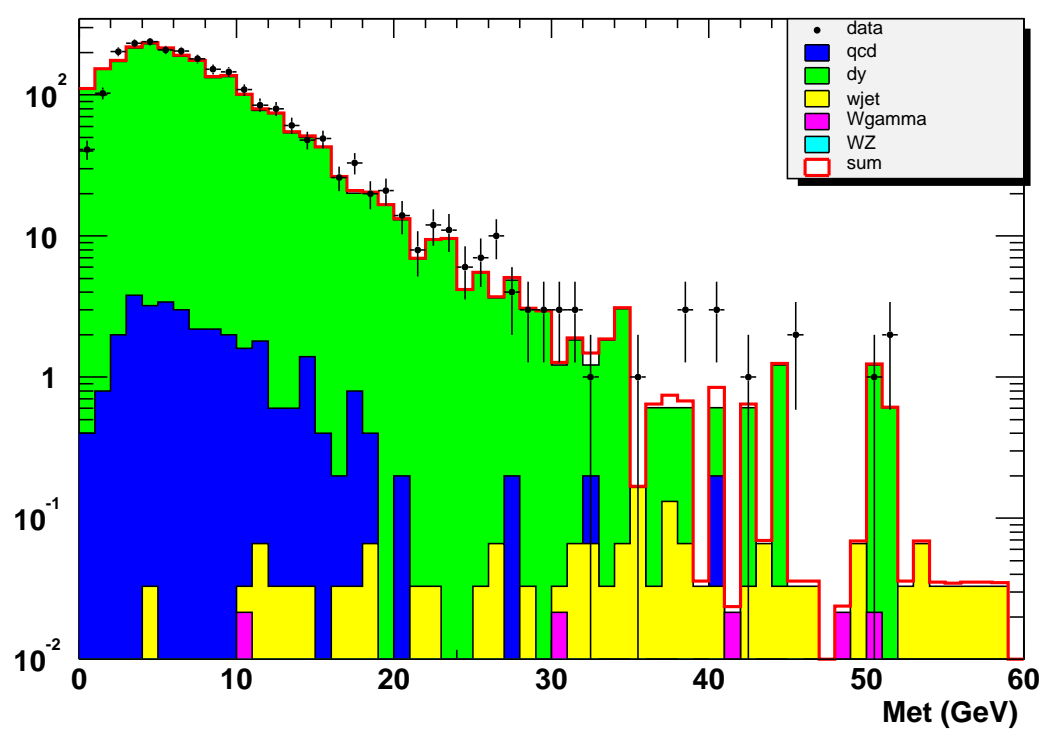

Figure 5.9: Missing $E_{T}$ distributions for central di-electron sample (with 2 central tight electrons). Data (in dots with error bars) and sum of backgrounds (solid line) are consistent. 
mass region $\left(M_{e e}>75 \mathrm{GeV} / \mathrm{c}^{2}\right)$. This under-estimation is caused by the larger charge mis-measurement rate for high-momentum electrons in data than in MC simulation. However, the discrepancy is far less important in our search for events with medium invariant mass $\left(20 \mathrm{GeV} / \mathrm{c}^{2}<M_{e e}<75 \mathrm{GeV} / \mathrm{c}^{2}\right)$, as shown by the good agreement between data and backgrounds after the invariant mass cut.

Overall, the data sample is well described by the backgrounds and we validate the data sample and the estimate of backgrounds used in this analysis.

\subsection{Summary}

After analyzing the data and estimating the background, we find that 1 event survived all the offline event selection cuts and the total number of background events passing the offline selection cuts is $1.59 \pm 0.44$. (Table 5.5). We observe no significant deviation of number of events from the expected background in the RunII data sample of $124.5 \mathrm{pb}^{-1}$. 


\begin{tabular}{|c|c|c|c|}
\hline \hline Cut & $Z / \gamma *$ & QCD & $W \gamma$ \\
\hline Di-electron Pair & $2374.44 \pm 254.07$ & $31.96 \pm 2.58$ & $0.11 \pm 0.05$ \\
Like-Sign Pair & $2.53 \pm 1.30$ & $12.86 \pm 1.62$ & $0.09 \pm 0.05$ \\
$20 \mathrm{GeV}<M_{e e}<75 \mathrm{GeV}$ & $0.00 \pm 0.01$ & $11.86 \pm 1.55$ & $0.07 \pm 0.04$ \\
$\not_{T}>15 \mathrm{GeV}$ & $0.00 \pm 0.01$ & $0.80 \pm 0.40$ & $0.07 \pm 0.04$ \\
\hline
\end{tabular}

\begin{tabular}{|c|c||c|c|}
\hline \hline WZ & W+jets & sum of bkdg & data \\
\hline $0.23 \pm 0.03$ & $1.99 \pm 0.39$ & $2408.73 \pm 254.08$ & 2349 \\
$0.11 \pm 0.01$ & $0.99 \pm 0.23$ & $16.58 \pm 2.09$ & 44 \\
$0.06 \pm 0.01$ & $0.75 \pm 0.19$ & $12.74 \pm 1.57$ & 11 \\
$0.05 \pm 0.01$ & $0.67 \pm 0.18$ & $1.59 \pm 0.44$ & 1 \\
\hline
\end{tabular}

Table 5.5: The number of events passing the cumulative cuts listed in the first column for the backgrounds and data sample (last column). The comparison between data and backgrounds is described in the text. 


\section{Chapter 6}

\section{Results and Conclusion}

In the previous chapter, we have shown that there exists no evidence in our data of excess of events in the like sign di-electron channel beyond the estimated background events in our data. In this chapter, we describe the final step in our analysis: the simulation of the expected SUSY signal and the extraction of cross section limits from the data.

\subsection{Simulation of Signal}

Although it is desirable to search for SUSY as model-independent as possible, it is necessary to resort to some specific model in order to reduce the number of free parameters to a phenomenologically tractable level. We have chosen the mSUGRA model for this purpose and a brief discussion was given in Section 1.3.3. To recapitulate, the 5 free parameters in mSUGRA are:

- $m_{0}$, the common scalar mass at the $M_{X}$ scale

- $m_{1 / 2}$, the common gaugino mass at the $M_{X}$ scale 
- $\tan (\beta)$, the ratio of the vacuum expectation values of the two Higgs doublets

- $A_{0}$, the common trilinear coupling constant at the $M_{X}$ scale

- $\operatorname{sign}(\mu)$, the sign of the Higgsino mass parameter $\mu$

In this analysis, $A_{0}$ is set to zero since it mainly affects the mass of the light stop, which is not considered here. $\operatorname{sign}(\mu)$ affects the mass difference of the gauginos. Positive $\mu$ will lead to smaller mass differences than negative $\mu$. These smaller mass differences result in less energetic leptons that are below the identification threshold of the $\mathrm{D} \emptyset$ detector. Therefore, $\mu$ is set negative in the analysis. $\tan (\beta)$ was excluded up to 2.4 by LEP's neutral higgs boson search in the framework of MSSM, so we choose $\tan (\beta)=2.5$. In most of the mSUGRA parameter space, the masses of $\tilde{\chi}_{1}^{ \pm}$and $\tilde{\chi}_{2}^{0}$ at weak scale are mainly determined by $m_{1 / 2}$ with the relationship of $m_{\tilde{\chi}_{1}^{ \pm}} \approx m_{\tilde{\chi}_{2}^{0}} \approx 0.8 m_{1 / 2}$ and are nearly independent of the value of $m_{0}$. Therefore, we choose $m_{0}=100 \mathrm{GeV} / \mathrm{c}^{2}$ and vary $m_{1 / 2}$ from $100 \mathrm{GeV} / \mathrm{c}^{2}$ to $140 \mathrm{GeV} / \mathrm{c}^{2}$ in steps of $10 \mathrm{GeV} / \mathrm{c}^{2}$. This set of parameters corresponds to chargino masses from $86 \mathrm{GeV} / \mathrm{c}^{2}$ to $115 \mathrm{GeV} / \mathrm{c}^{2}$, which is around the mass limit $\left(m_{\tilde{\chi}_{1}^{ \pm}}>103.5 \mathrm{GeV} / \mathrm{c}^{2}\right)$ set by the LEP chargino searches [55].

We use PYTHIA to generate the mSUGRA events. However, PYTHIA does not support generating mSUGRA events directly, one has to use ISAJET's Renormalization Group Equations (RGE's) programs to evolve the SUSY parameters from the unification scale to the weak scale and then supply the SUSY parameters to PYTHIA. The New Phenomena Group [54] at DØ developed 
a tool [56] that uses ISAJET to solve the RGE's and then output the SUSY parameters in the format accepted by PYTHIA. We save these SUSY parameters in a PYTHIA card file and then add the allowed production and decay modes of $\tilde{\chi}_{1}^{ \pm}$and $\tilde{\chi}_{2}^{0}$. With a complete card file, we run the MC_RUNJOB [57] program to generate a desired amount of MC events.

The cross section of these events are first taken from PYTHIA and then corrected with a K-factor to get the next-to-leading order (NLO) cross section. The K-factors of the $\tilde{\chi}_{1}^{ \pm} \tilde{\chi}_{2}^{0}$ production at Tevatron range from 1.30 to 1.15 for chargino mass between $70 \mathrm{GeV} / \mathrm{c}^{2}$ and $280 \mathrm{GeV} / \mathrm{c}^{2}$ [58]. For chargino masses around $100 \mathrm{GeV} / \mathrm{c}^{2}$, we use a flat K-factor 1.25 .

Table 6.1 shows the five sets of simulated mSUGRA mass points and their corresponding $m\left(\tilde{\chi}_{2}^{0}\right), m\left(\tilde{\chi}_{1}^{ \pm}\right)$, branching ratio's, NLO cross sections and the numbers of generated events.

\subsection{Signal Efficiency}

The procedure for the determination of signal efficiency is the same as that for the Standard Model background and was described in Chapter 5. The detection efficiencies for the five mSUGRA points are plotted in Figure 6.1. As one can see, the detection efficiency increases with the chargino mass. This is readily explained: since $m_{\tilde{\chi}_{1}^{ \pm}} \approx m_{\tilde{\chi}_{2}^{0}} \approx 2 m_{\tilde{\chi}_{1}^{0}}$, higher $\tilde{\chi}_{1}^{ \pm}$and $\tilde{\chi}_{2}^{0}$ masses produce electrons that are more energetic on average, and which pass our signal selection cuts more frequently.

Table 6.2 shows, for each mass point, the chargino mass, the signal detec- 


\begin{tabular}{|l|l|l|l|l|l|l|l|}
\hline \hline set & $m_{1 / 2}$ & $m\left(\tilde{\chi}_{2}^{0}\right)$ & $m\left(\tilde{\chi}_{1}^{ \pm}\right)$ & B.R. & B.R. & $\sigma \times$ B.R. & N.Evts \\
& $\left(\mathrm{GeV} / \mathrm{c}^{2}\right)$ & $\left(\mathrm{GeV} / \mathrm{c}^{2}\right)$ & $\left(\mathrm{GeV} / \mathrm{c}^{2}\right)$ & $\left(\tilde{\chi}_{2}^{0} \rightarrow e e \tilde{\chi}_{1}^{0}\right)$ & $\left(\tilde{\chi}_{1}^{ \pm} \rightarrow e \nu \tilde{\chi}_{1}^{0}\right)$ & $(\mathrm{pb})$ & \\
\hline \hline 1 & 100 & 86.1 & 86.9 & $14.0 \%$ & $11.4 \%$ & 0.031 & 6000 \\
\hline 2 & 110 & 93.3 & 93.9 & $13.0 \%$ & $14.2 \%$ & 0.025 & 5000 \\
\hline 3 & 120 & 100.3 & 100.8 & $12.3 \%$ & $15.2 \%$ & 0.018 & 6000 \\
\hline 4 & 130 & 107.5 & 108.0 & $11.5 \%$ & $15.8 \%$ & 0.014 & 6000 \\
\hline 5 & 140 & 114.7 & 115.1 & $10.8 \%$ & $16.1 \%$ & 0.010 & 7000 \\
\hline \hline
\end{tabular}

Table 6.1: Chargino and Neutralino's masses and branching ratios for the five mSUGRA points. The remaining mSUGRA parameters are $m_{0}=100 \mathrm{GeV} / \mathrm{c}^{2}$, $\tan \beta=2.5, A_{0}=0$, and $\operatorname{sign}(\mu)=-1$.

tion efficiency $\epsilon_{d e t}$, and the number of expected events in the data sample of $124.5 \mathrm{pb}^{-1}$. The signal detection efficiencies range from $4.2 \%$ to $6.4 \%$. Taking mSUGRA Set 1 as an example, I explain how each selection cut reduces the signal efficiency in the following. In mSUGRA set 1, about $35 \%$ of the events have two loose central electrons with $E_{T}>15 \mathrm{GeV}$ and $10 \mathrm{GeV}$ respectively. However, when we require both electrons to have track matches, only $\sim 15 \%$ of the events survive. This is mainly caused by the relatively low tracking efficiency $72.4 \%$ (Section 5.1.2) and its effect is more obvious ( $\sim 50 \%$ loss) when we need two track matches in this analysis. In the remaining di-electron sample, the like-sign requirement reduces efficiency to $7.2 \%$; the invariant mass cut reduces the efficiency to $5.5 \%$; and the missing $\#_{T}$ cut reduces the overall efficiency to $4.2 \%$. 


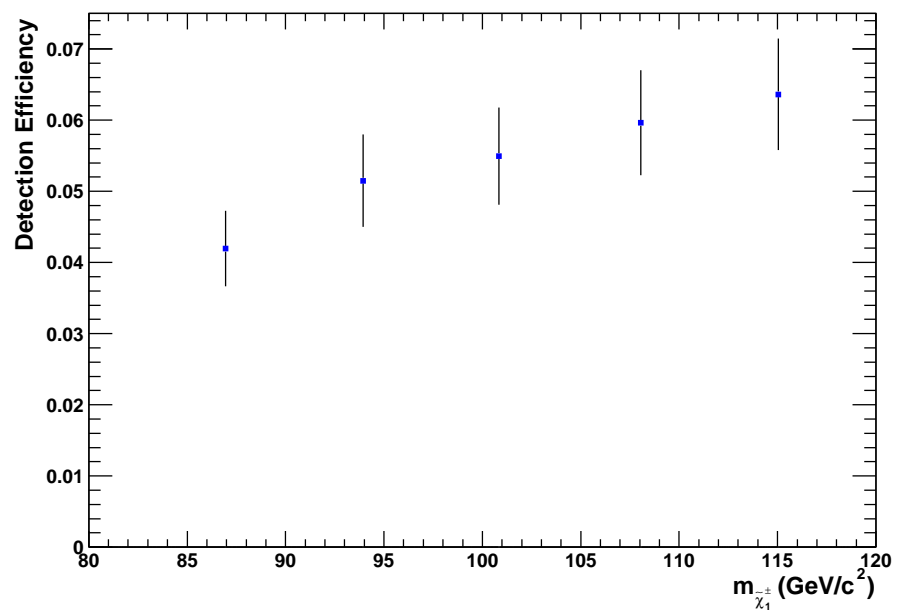

Figure 6.1: Detection efficiency for $\tilde{\chi}_{1}^{ \pm} \tilde{\chi}_{2}^{0}$ pair as a function of chargino mass.

\subsection{Extraction of Cross Section Limits}

In the data sample, which corresponds to an integrated luminosity of 124.5 $\mathrm{pb}^{-1}$, we find no significant excess of events over the background. Thus, the data has been used to extract the upper limit of the cross section at the $95 \%$ confidence for the five mSUGRA points we are studying. For each point, the upper limit of the cross section was obtained using the estimated signal efficiencies and errors, the estimated number of background events and its error, the actual number of events observed in the data and the total integrated luminosity. There are two major approaches to extract the upper limit, the Bayesian approach and the Classical (Frequentist) approach. For this analysis, 


\begin{tabular}{|c|c|c|c|}
\hline \hline Set & $m_{\tilde{\chi}_{1}^{ \pm}} \mathrm{GeV} / \mathrm{c}^{2}$ & $\epsilon_{\text {det }}(\%)$ & $N_{\text {exp }} \pm \delta N$ \\
\hline \hline 1 & 86.9 & $4.2 \pm 0.5$ & $0.16 \pm 0.02$ \\
\hline 2 & 93.9 & $5.1 \pm 0.6$ & $0.16 \pm 0.02$ \\
\hline 3 & 100.8 & $5.4 \pm 0.7$ & $0.12 \pm 0.01$ \\
\hline 4 & 108.0 & $6.0 \pm 0.7$ & $0.10 \pm 0.01$ \\
\hline 5 & 115.1 & $6.4 \pm 0.8$ & $0.08 \pm 0.01$ \\
\hline \hline
\end{tabular}

Table 6.2: Chargino masses, signal detection efficiencies, and the number of expected events in $124.5 \mathrm{pb}^{-1}$ for the five mSUGRA points. 
we use the Bayesian approach. A brief overview of the Bayesian approach is presented in the following [59]:

The Bayesian theorem relates the prior probability to the posterior probability:

$$
P(A \mid B C)=\frac{P(B \mid A C) P(A \mid C)}{P(B \mid C)}
$$

where $P(A \mid B)$ denotes the probability $P$ of the proposition A to be true, given that proposition $\mathrm{B}$ is true. $C$ represents all relevant prior knowledge.

Here the denominator is determined by the normalization condition

$$
\sum_{\text {AllA }} P(A \mid B C)=1
$$

In case of a continuous variable $x, P(x \mid B) d x$ is the probability of $x$ to have a value between $x$ and $x+d x$, given that proposition $\mathrm{B}$ is true.

To find the limit, the first step is to define a model. For a counting experiment such as used in this analysis, the expected number of events $\mu$ is related to the signal cross section $\sigma$, the signal detection efficiency $\epsilon$, the integrated luminosity $\mathcal{L}$, and the expected background $b$ as:

$$
\mu=b+\mathcal{L} \epsilon \sigma
$$

where the first term on the right is the expected number of background events and the second term is the expected number of signal events. In the case of a counting experiment, the probability of observing $k$ events in the data, given an expectation value of $\mu$, is given by the Poisson likelihood function: 


$$
P(k \mid \mu, I)=\frac{e^{-\mu} \mu^{k}}{k !}
$$

where $I$ denotes all the relevant prior information in the problem. From equation 6.3 and equation 6.4 , one can write

$$
P(k \mid \sigma, \mathcal{L}, \epsilon, b, I)=\frac{e^{-(b+\mathcal{L} \epsilon \sigma)}(b+\mathcal{L} \epsilon \sigma)^{k}}{k !}
$$

The next step will be to assign the prior probabilities for all parameters. Assuming the parameters $\sigma, \mathcal{L}, \epsilon$, and $b$ to be independent and uncorrelated, the prior probability can be factorized as:

$$
P(\sigma, \mathcal{L}, \epsilon, b \mid I)=P(\sigma \mid I) P(\mathcal{L}, \epsilon, b \mid I)
$$

For the signal cross section, a flat prior probability is taken

$$
P(\sigma \mid I)= \begin{cases}1 / \sigma_{\max } & \text { if } 0 \leq \sigma \leq \sigma_{\max } \\ 0 & \text { otherwise }\end{cases}
$$

As for $\mathcal{L}, \epsilon$ and $b$, we have estimation of both their mean values and the errors on them; for all of these Gaussian prior probabilities are assumed. From equation 6.6 and our assumption of the prior, Bayes's theorem for our problem becomes:

$$
P(\sigma, \mathcal{L}, \epsilon, b \mid k, I) \propto \frac{e^{-(b+\mathcal{L} \epsilon \sigma)}(b+\mathcal{L} \epsilon \sigma)^{k}}{k !} P(\sigma \mid I) P(\mathcal{L}, \epsilon, b \mid I)
$$

where the constant of proportionality is determined by the condition 


$$
\int_{0}^{\infty} d \sigma \int_{0}^{\infty} d \mathcal{L} \int_{0}^{1} d \epsilon \int_{0}^{\infty} d b P(\sigma, \mathcal{L}, \epsilon, b \mid k, I)=1
$$

Since we are only interested in $\sigma$, we integrate equation over the parameters $\mathcal{L}, \sigma$ and $b$ to get the posterior probability distribution for $\sigma$

$$
P(\sigma \mid k, I)=\int_{0}^{\infty} d \mathcal{L} \int_{0}^{1} d \epsilon \int_{0}^{\infty} d b P(\sigma, \mathcal{L}, \epsilon, b \mid k, I)
$$

The $100 \times C \%$ confidence level upper limit on the cross section $\sigma_{U L}$ is defined by

$$
C=\int_{0}^{\sigma_{U L}} d \sigma P(\sigma \mid k, I)
$$

The $95 \%$ confidence level corresponds to $C=0.95$.

Here, one interesting point to note is that if the posterior distribution for $\sigma$ peaks significantly away from zero, it may be considered as a discovery.

\subsection{Cross Section Limits}

We used a macro [60] implementing the Bayesian method and extracted the 95\% confidence level upper limits for the five mSUGRA points. In Figure 6.2, we plot the upper limits of the $\tilde{\chi}_{1}^{ \pm} \tilde{\chi}_{2}^{0}$ production cross section times branching ratio to tri-electron against the chargino mass and link them with a curve. The cross sections above the curve are excluded by this analysis. They range from $0.79 \mathrm{pb}$ at $m_{\tilde{\chi}_{1}^{ \pm}}=86.9 \mathrm{GeV} / \mathrm{c}^{2}$ to $0.52 \mathrm{pb}$ at $m_{\tilde{\chi}_{1}^{ \pm}}=115.1 \mathrm{GeV} / \mathrm{c}^{2}$. For comparison, we also plot the area excluded $\left(m_{\tilde{\chi}_{1}^{ \pm}}<103.5 \mathrm{GeV} / \mathrm{c}^{2}\right)$ by the LEP 
chargino searches. In Table 6.3, we list the $\sigma \times$ B.R. $(3 \mathrm{e}+\mathrm{X})$ upper limits for all five mSUGRA mass points.

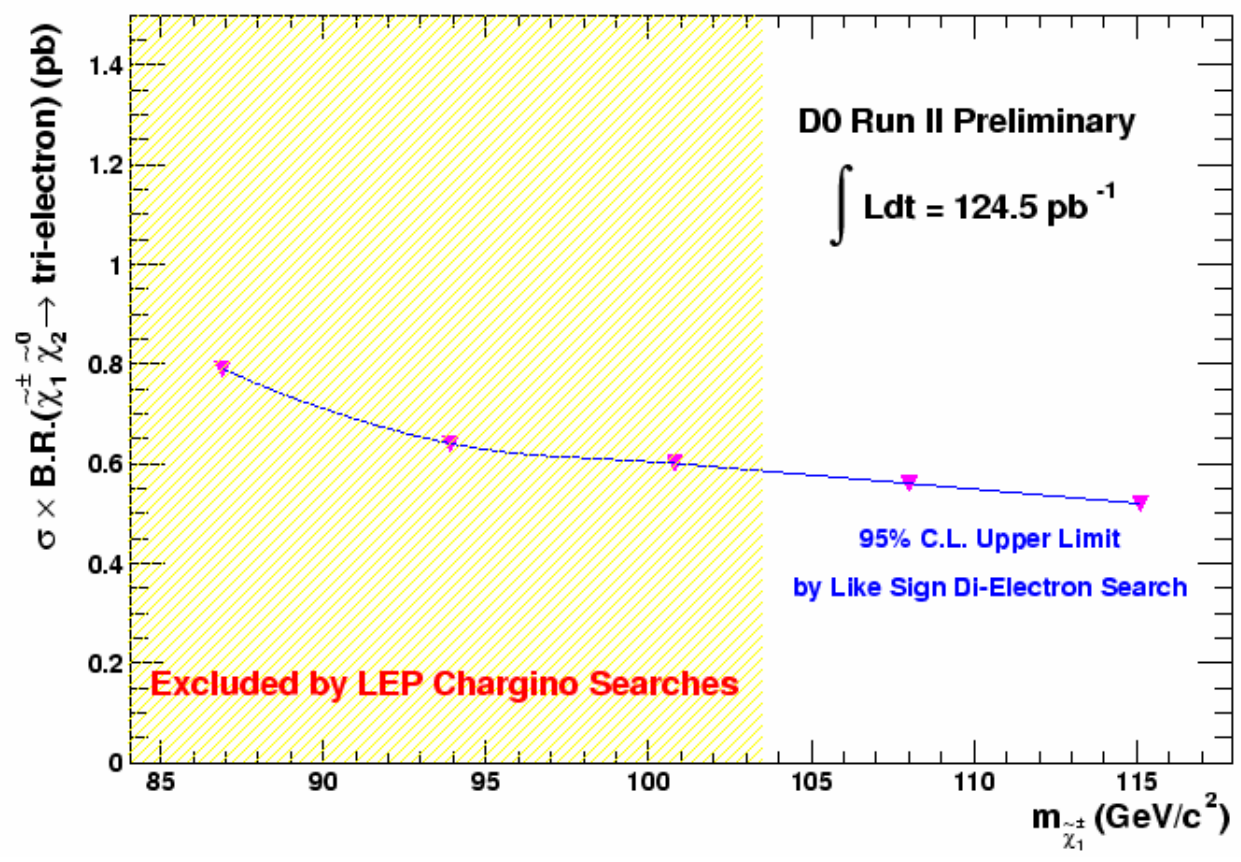

Figure 6.2: The solid curve is the $95 \%$ C.L. upper limit of the $\tilde{\chi}_{1}^{ \pm} \tilde{\chi}_{2}^{0}$ production cross section times branching ratio to tri-electron. The hatched area up to 103.5 $\mathrm{GeV} / \mathrm{c}^{2}$ was excluded by the LEP chargino searches. 


\begin{tabular}{|c|c|c|}
\hline \hline Set & $m_{\tilde{\chi}_{1}^{ \pm}}\left(\mathrm{GeV} / \mathrm{c}^{2}\right)$ & $\sigma_{U . L .} \times$ B.R. $(3 e+X)(\mathrm{pb})$ \\
\hline \hline 1 & 86.9 & 0.79 \\
\hline 2 & 93.9 & 0.64 \\
\hline 3 & 100.8 & 0.60 \\
\hline 4 & 108.0 & 0.56 \\
\hline 5 & 115.1 & 0.52 \\
\hline \hline
\end{tabular}

Table 6.3: Cross section times Branching ratio upper limit the 5 mSUGRA points 


\subsection{Future Improvement}

This analysis is the first attempt at $\mathrm{D} \varnothing$ to search for $\tilde{\chi}_{1}^{ \pm} \tilde{\chi}_{2}^{0}$ pair production in the like-sign di-lepton channel with the upgraded detector as a complement to the tri-lepton channel. We see that the replacement of the requirement of the third electron by the like-sign requirement has achieved reasonable signal detection efficiency and backgrounds control. In addition, the signal detection efficiency will be further increased as DØ continues improving its tracking efficiency. The use of a multi-variate method for offline event selection may also be helpful.

The progress in the DØ's fast Monte Carlo simulation programs [61] (PMCS) will allow us to generate larger samples of SUSY events without going through the full detector simulation. It will make a scan in the SUSY parameter space possible.

The ongoing analysis in the like-sign di-muon channel [62] and future combination of the like-sign electron/muon channel will make the like-sign di-lepton search more complete. Ultimately, the greatest sensitivity to the chargino and neutralino search will be achieved by combining the the like-sign di-lepton channel with the tri-lepton channel. 


\section{Appendix A}

\section{Electron Identification Efficiency}

\section{A.1 Loose Electron ID Efficiency}

The EMID efficiency in the CC region is measured with the "tag and probe" method using events from $Z \rightarrow e e$ decays. We start with events with two "probe" electrons satisfying the following criteria: 1. Both probes have $E_{T}>25 \mathrm{GeV}$ and are in the fiducial region; 2. At least one of the probes passes tight cuts ("tag") of EM fraction $>0.9$, isolation $<0.15, H M x 8<20$ and has a matched track.

The EMID efficiency can be computed by:

$$
\epsilon_{c u t}=\frac{2(t t)+(t p)}{2(t t)+(t p)+(t f)}
$$

where

- $t t=$ numbers of events where both electrons pass the tight cuts (therefore pass the cuts under study);

- $t p=$ number of events where one electron passes the tight cuts and the 
other passes the cuts under study but fails the tight cuts;

- $t f=$ number of events where one electron passes the tight cuts and the other electron fails the cuts under study (and therefore fails the tight cuts as well).

Here the set of cuts under study is: EMfraction > $>0.9$, isolation < $0.15, H M x 8<20$

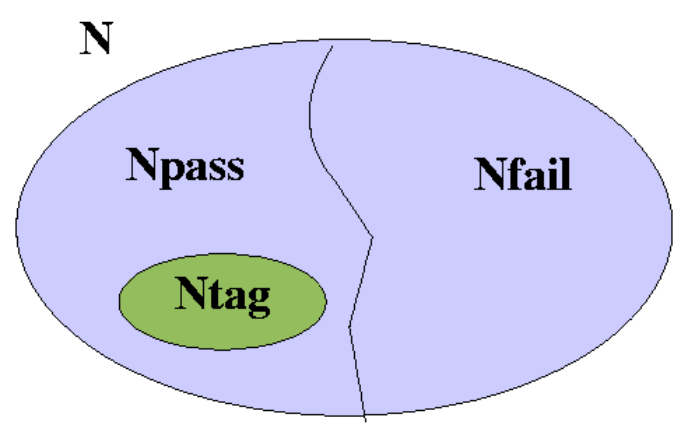

Figure A.1: Illustration of sample sets used to measure the EMID efficiency.

It is not obvious that Equation A.1 indeed represents the EMID efficiency, we verify it as follows. The total number of probe electrons $N$ can be divided into $N_{\text {pass }}$ and $N_{\text {fail }}$, with $N_{\text {tag }}$ a subset of $N_{\text {pass }}$, as illustrated in Figure A.1. With these definitions, the following relations hold:

- $\epsilon_{\text {tag }}=N_{\text {tag }} / N$

- $\epsilon_{\text {pass }}=N_{\text {pass }} / N$

- $\epsilon_{\text {fail }}=N_{\text {fail }} / N=\left(N-N_{\text {pass }}\right) / N=1-\epsilon_{\text {pass }}$ 
- $\epsilon_{\text {pass(but-fail-tag })}=\left(N_{\text {pass }}-N_{\text {tag }}\right) / N=\epsilon_{\text {pass }}-\epsilon_{\text {tag }}$

Assuming there are no correlations between the electrons, we can use the above relations to calculate following efficiencies:

- $\epsilon_{t t}=\epsilon_{t a g}^{2}$

- $\epsilon_{t p}=2 \epsilon_{t a g}\left(\epsilon_{\text {pass }}-\epsilon_{t a g}\right)$

- $\epsilon_{t f}=2 \epsilon_{t a g}\left(1-\epsilon_{\text {pass }}\right)$

Therefore, Equation A.1 reduces to:

$$
\begin{aligned}
\epsilon_{c u t} & =\frac{2(t t)+(t p)}{2(t t)+(t p)+(t f)}=\frac{2 \epsilon_{\text {tag }}^{2}+2 \epsilon_{\text {tag }}\left(\epsilon_{\text {pass }}-\epsilon_{\text {tag }}\right)}{2 \epsilon_{\text {tag }}^{2}+2 \epsilon_{\text {tag }}\left(\epsilon_{\text {pass }}-\epsilon_{\text {tag }}\right)+2 \epsilon_{\text {tag }}\left(1-\epsilon_{\text {pass }}\right)} \\
& =\frac{2 \epsilon_{\text {tag }} \epsilon_{\text {pass }}}{2 \epsilon_{\text {tag }} \epsilon_{\text {pass }}+2 \epsilon_{\text {tag }}-2 \epsilon_{\text {tag }} \epsilon_{\text {pass }}}=\epsilon_{\text {pass }}
\end{aligned}
$$

where $\epsilon_{\text {pass }}$ is indeed the efficiency of our cut, i.e. the number of electrons passing the cuts under study divided by the total number of probe electrons. The advantage of this algorithm is that it counts the number of events rather than the number of electrons. Therefore, it allows us to subtract non- $Z$ backgrounds from the sample and use nearly pure $Z$ events. Figure A.2 shows the invariant mass distribution of events satisfying the $2(t t)+(t p)$ criteria and events satisfying the $2(t t)+(t p)+(t f)$ criteria. The backgrounds are also shown in the plots.

The numerator in Equation A.1 is then the number of entries between 80 and $100 \mathrm{GeV}$ after subtracting background in the $2(t t)+(t p)$ plot. Similarly, the denominator in Equation A.1 is then the number of entries between 80 and 

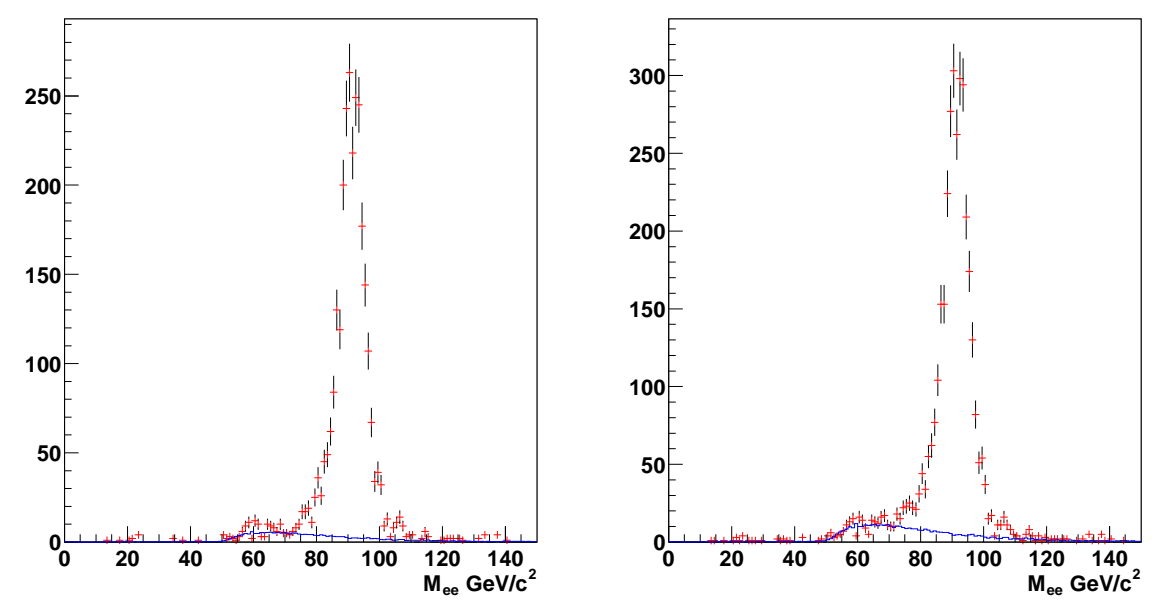

Figure A.2: The 2(tt)+(tp) (left) and $2(\mathrm{tt})+(\mathrm{tp})+(\mathrm{tf})$ (right) distributions for events with $2 \mathrm{CC}$ electrons. The points are data and the lines are the expected non- $Z$ background.

$100 \mathrm{GeV}$ after subtracting background in the $2(t t)+(t p)+(t f)$ plot. Here we have applied a mass window cut $(80-100 \mathrm{GeV})$ to further purify the $Z$ events.

The measured EMID efficiencies in data and MC are $\epsilon_{C C}^{\text {data }}=85.0 \pm 2.0 \%$ and $\epsilon_{C C}^{M C}=96.5 \pm 2.0 \%$ respectively. A correction factor of $0.88 \pm 0.03$ is applied for MC electrons to correct the discrepancy.

\section{A.2 Track Matching Efficiency}

The track matching efficiency $\epsilon_{t r k}$ for CC electrons is measured using events with 2 loose electrons with $E_{T}>25 \mathrm{GeV}$. The tracking efficiency is calculated as the ratio of the number of electrons in the $\mathrm{Z}$ peak $(80-100 \mathrm{GeV})$ that have track match to the total number of electrons in the $\mathrm{Z}$ peak (twice the number 
of Z events).

Let $N_{0}, N_{1}$ and $N_{2}$ denote the number of $\mathrm{Z}$ events in which none, one or both electrons have a matching track respectively, the tracking efficiency can be expressed by:

$$
\epsilon_{t r k}=\frac{N_{1}+2 N_{2}}{2\left(N_{0}+N_{1}+N_{2}\right)}
$$

We plot the combined distribution of $N_{1}+2 N_{2}$ and $N_{0}+N_{1}+N_{2}$ in data in Figure A.3. The numerator in Equation A.2 is the number of entries between 80 and $100 \mathrm{GeV}$ after subtracting background in the $N_{1}+2 N_{2}$ plot. Similarly, the denominator in Equation A.2 is the number of entries between 80 and 100 $\mathrm{GeV}$ after subtracting background in the $2\left(N_{0}+N_{1}+N_{2}\right)$ plot.
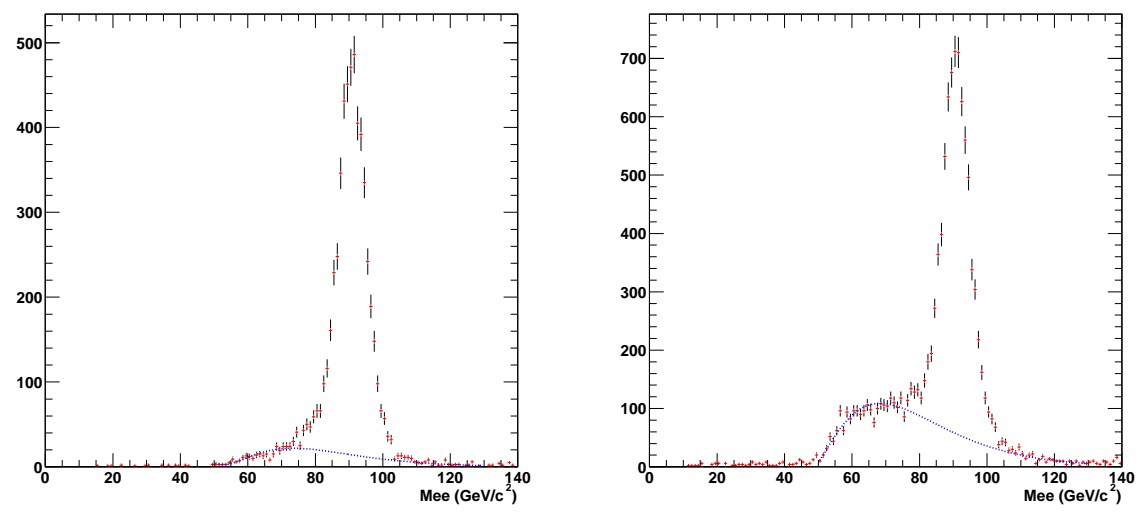

Figure A.3: The $N_{1}+2 N_{2}$ (left) and $N_{0}+N_{1}+N_{2}$ (right) distributions for events with $2 \mathrm{CC}$ electrons in data. The points are data and the lines represent background.

The tracking efficiencies in data and MC are measured to be $\epsilon_{\text {trk }}^{\text {data }}=72.4 \pm$ 
$0.5 \%$ and $\epsilon_{t r k}^{\text {data }}=87.4 \pm 0.4 \%$ respectively. A correction factor of $0.828 \pm 0.008$ is applied to correct the discrepancy. 


\section{Bibliography}

[1] Donald H. Perkins, Introduction to High Energy Physics, Addison-Wesley, 1999.

[2] B. Lee, C. Quigg and H. Thacker, Phys. Rev. D16, 1519 (1977).

[3] E. Fahri and L. Susskind, Phys. Rep. 74, 277 (1981).

[4] Xerxes Tata, What is SUSY and how do we find it?, hep-ph/970630 (1997).

[5] J. Wess and J. Bagger, Supersymmetry and Supergravity, Princeton University Press, 1983.

[6] A.H. Chamseddine, R. Arnowitt and P. Nath, Phys. Rev. Lett. 29, 970 (1982); R. Barbieri, S. Ferrara and C.A. Savoy, Phys. Lett. B119, 343 (1982); L. Hall, J. Lykken and S. Weinberg, Phys. Rev. D27, 2359 (1983); P. Nath, R. Arnowitt and A.H. Chamseddine, Nucl. Phys. B227, 121 (1983).

[7] Report of SUGRA Working Group for Run II of the Tevatron, hepph/0003154 (2000). 
[8] H. Baer and X. Tata, Phys. Rev. D 47, 2739 (1993)

[9] J.Nachtman, et al., Study of a Like-Sign Dilepton Search for CharginoNeutralino Production at CDF, hep-ex/9902010 (1999).

[10] Manuel Drees, An Introduction to Supersymmetry, hep-ph/9611409 (1996).

[11] J. Thompson, Introduction to Colliding Beams at Fermilab, FERMILABTM-1909, 1994.

[12] FermiLab, Tevatron Run II Handbook, Internal FNAL note (unpublished).

[13] D.E.Johnson, Instrumentation Requirements for the Fermilab Main Injector", Internal Main Injector Note 76, 1992 (unpublished).

[14] DØ Collaboration, DØ Upgrade: the Detector and its Physics, Fermilab Pub-96/357-E.

[15] DØ Collaboration, Nucl. Instr. Meth. A338, 185 (1994).

[16] Conceptual Design of a 2 Tesla Superconducting Solenoid for the Fermilab DØ Detector Upgrade, Fermilab TM-1886/DØ Note 2167.

[17] DØ Collaboration, DØ Silicon Tracker Technical Design Report, DØ Note 2169.

[18] The DØ Collaborration, The DØ Upgrade Central Fiber Tracker, http://d0server1.fnal.gov/users/stefan/www/CFT_TDR/CFT_TDR.ps. 
[19] D. Adams et al., Nucl. Phys. B44, 332 (1995); IEEE Trans. Nucl. Sci., Vol 43, No.3, 1146 (1996).

[20] A Litter Tour of VLPC's, http://d0server1.fnal.gov/users/stefan/www/vlpc/index.html.

[21] Design Report of the Central Preshower Detector for the DØ Upgrade, DØ Note 3014.

[22] A. Gordeev et al., Technical Design Report of the Forward Preshower Detector for the DØ detector, DØ note 3445

[23] The DØ Calorimeter Electronics Group, Calorimeter Electronics Upgrade for Run 2, http://www-d0.fnal.gov/hardware/cal/.

[24] Y. Arnoud, http://www-d0.fnal.gov/phys_id/emid/d0_private/minutes/ 20030409yannick.pdf.

[25] Technical Design Report for the Upgrade of the ICD for DØ Run II, DØ Note 2686.

[26] B.Baldin et al., Technical Design of the Central Muon System, DØ Note 3365; G. Alexeev et al., Technical Design of the Forward Muon Tracking Detector Based on Mini-Drift Tubes, DØ Note 3366.

[27] G. Blazey, The DØ Run II Trigger, 10th IEEE Real-Time Comp. App. in Nuclear, Particle, and Plasma Physics, Beaue, France, Conf. Proc. (1997).

[28] The DØReco Program, http://www-d0.fnal.gov/computing/algorithms/ howto/howtoreco.html. 
[29] D. Adams, Finding Tracks, DØ Note 2958-2 (1998); DØ Tracking Group, Tracking Algorithms, http://www-d0.fnal.gov/global_tracking/.

[30] A. Khanov, HTF: histogramming method for finding tracks, DØ Note 3778 $(2000)$

[31] M. Narain and F. Stichelbaut, Vertex Reconstruction using the Impact Parameters Technique, DØ Note 2560 (1999).

[32] N. Hadley, Cone Algorithm for Jet Finding, DØ Note 904 (1989).

[33] http://www-d0.fnal.gov/d0dist/dist/releases/p13.06.01/calreco/

[34] L. Duflot, G. Le Meur, F. Touze, The ConeClusterAlgo User Guide, http://www-d0.fnal.gov/d0dist/dist/releases/p13.06.01/calreco/ ConeAlgo.ps

[35] L. Duflot and M. Ridel, The CellNN algorithm: cell level clustering in the DØ calorimeter DØ Note 3923 (2001)

[36] T. Ferbel, Experimental Techniques in High Energy, Nuclear, and Particle Physics, World Scientific, page 6 (1991)

[37] The em_util packgae, http://www-d0.fnal.gov/d0dist/dist/packages/em_util/ p13-br-03/

[38] J. Coss et al., Jet Energy Scale and Resolution for p13 data and MC, D Note 4115 (2003). 
[39] Jet Energy Scale and Resolution, http://www-d0.fnal.gov/phys_id/ jes/d0_private/jes.html.

[40] DØ Luminosity Group, http://www-d0.fnal.gov/phys_id/luminosity/data -access/

[41] U. Bluemenschein and V. Bueshcer, Search for the Associated Production of Chargino and Neutralino in Trilepton Final States, DØ Note 4245 (2003).

[42] F. Paige and S. Protopopescu, Brookhaven National Laboratory Report No. 38304 (1986).

[43] T. Sjöstrand, L. Lönnblad, S. Mrenna and P. Skands, PYTHIA 6.206, hep-ph/0108264.

[44] G. Marchesini, B. Webber, G. Abbiendi, I. Knowels, M. Seymour, Computer Physics Communications 67, 465 (1992).

[45] http://www-d0.fnal.gov/d0dist/dist/packages/cardfiles.

[46] R.D. Field, R.P. Feynman, Nucl. Phys. B136, 1 (1978).

[47] B. Anderson, G. Gustafson, G. Ingelman, and T. Sjőstrand, Phys. Rep. 97, 33 (1983).

[48] http://www-d0.fnal.gov/d0dist/dist/releases/test/d0gstar/docs/html /d0gstar.html;

[49] http://www-d0.fnal.gov/computing/MonteCarlo/simulation/d0sim.html. 
[50] F. Carminati et al, GEANT Users Guide, CERN Program Library, 1991.

[51] EMID certification, http://www-d0.fnal.gov/phys_id/emid/d0_private/ certification/welcome.html

[52] private communication with Junjie Zhu

[53] DØ Event Display Program, http://www-d0.fnal.gov/computing/graphics/d0graphics.html.

[54] http://www-d0.fnal.gov/Run2Physics/np/

[55] LEP SUSY working group, http://lepsusy.web.cern.ch/lepsusy/www/ inos_moriond01/charginos_pub.html

[56] http://www-d0.fnal.gov/Run2Physics/np/d0_private/mc/SUSY_scans.html

[57] http://www-clued0.fnal.gov/runjob

[58] W. Beenakker et al., The production of charginos/neutralinos and sleptons at hadron colliders, hep-ph/9906298 (1999).

[59] I. Bertam et al., A Recipe for the Construction of Confidence Limits, D0 Note 3476 (1998).

[60] G. Landsberg, Private Communication

[61] http://www-d0.fnal.gov/computing/MonteCarlo/pmcs/pmcs_doc/pmcs.html

[62] A. Yurkewicz et al., Search for mSUGRA SUSY in the like-sign dimuon channel, http://www-d0.fnal.gov/Run2Physics/np/d0_private/ results/Moriond_2004/Adam.pdf 